Electronic Journal of Statistics

Vol. 15 (2021) 3249-3286

ISSN: $1935-7524$

https://doi.org/10.1214/21-EJS1860

\title{
An inverse norm weight spatial sign test for high-dimensional directional data*
}

\author{
Hongfei Wang \\ School of Mathematics and Statistics and KLAS of MOE, Northeast Normal University \\ e-mail: wanghf206@nenu.edu.cn \\ Long Feng ${ }^{\dagger}$ \\ School of Mathematics and Statistics and KLAS of MOE, Northeast Normal University \\ e-mail: ${ }^{\dagger}$ fengl100@nenu.edu.cn

\section{Binghui Liu} \\ School of Mathematics and Statistics and KLAS of MOE, Northeast Normal University \\ e-mail: liubh100@nenu.edu.cn

\section{Qin Zhou} \\ School of Mathematics and Statistics, Jiangsu Normal University \\ e-mail: graceqinzhou@jsnu.edu.cn

\begin{abstract}
In this paper, we focus on the high-dimensional location testing problem of directional data under the assumption of rotationally symmetric distributions, where the data dimension is potentially much larger than the sample size. We study the family of directional weighted spatial sign tests for this testing problem and establish the asymptotic null distributions and local power properties of this family. In particular, we find that the test based on the inverse norm weight, named as the inverse norm weight spatial sign test, has the maximum asymptotic power in this family. As demonstrated by extensive numerical results, the inverse norm weight spatial sign test has advantages in empirical power compared with some other members in the family as well as some existing tests.
\end{abstract}

MSC2020 subject classifications: $62 \mathrm{G} 10$.

Keywords and phrases: Directional data, high dimension, location test, rotationally symmetric distribution, weighted spatial sign.

Received December 2020.

\section{Introduction}

Directional data have been widely studied in meteorology [7], astronomy [2], earth science [22] and biology [10]. These fields naturally yield a large number of directional data, such as the wind direction data and the earth scale spatial

${ }^{*}$ This work was supported by NSFC grants 11501092, 11571068, 11671073, 11671-178, the Fundamental Research Funds for the Central Universities grant 241201-7BJ002, the Key Laboratory of Applied Statistics of MOE (KLAS) grants 130026507 and 130028612.

${ }^{\dagger}$ Corresponding author. 
data, which are commonly considered as an implementation of a random vector on a hypersphere. Besides, some data from other fields are essential only for their relative size, hence being projected onto a unit hypersphere, where the projection removes the overall scale factor associated with the size. For analyzing directional data, the family of rotationally symmetric distributions is one of the most commonly used distributions, which has played a central role in the application of directional statistics [15]. One prominent member of the family of rotationally symmetric distributions is the Fisher-von Mises-Langevin (FvML) distribution, which plays a critical role in directional statistics, on par with that of the Gaussian distribution in the classic multivariate setting.

The literature on the inference of location parameters under the assumption of rotationally symmetric distributions is abundant. For example, [23], [3], [11], [13] and [19] considered the location inference problems in low-dimensional situations. [21] and [5] tackled the problem for spherical regression, while [1] considered the location testing problem under axial frames. [8] considered the problem of testing rotational symmetry on hyperspheres, and introduced two locally asymptotically maximin tests against two classes of directional distributions in the Le Cam sense.

In this paper, we focus on the high-dimensional location testing problem of directional data under the assumption of rotationally symmetric distributions, where the null hypothesis is that the location parameter vector is equal to a given vector on the unit hypersphere. To deal with such a testing problem, [23] proposed a traditional Watson test based on the mean of the spherical sample. [17] proposed a class of rank tests and discussed the Le Cam optimality of the tests. Then, [12] proposed a high-dimensional Watson test by standardizing the traditional Watson statistic.

To develop some alternative high-dimensional tools for testing location under rotationally symmetric distributions, in this paper we study the family of directional weighted spatial sign tests for testing location of directional data under rotationally symmetric distributions. In particular, we find that the test based on the inverse norm weight, named as the inverse norm weight spatial sign test, has the maximum asymptotic power in this family. Indeed, the proposed inverse norm weight spatial sign test is an extension of the inverse norm sign test devised for general data. We then present its asymptotic properties under the unified framework of directional weighted spatial sign tests, and demonstrate its empirical power advantages via extensive numerical results.

The rest of the paper is organized as follows. In Section 2, we introduce the family of directional weighted spatial sign tests for high-dimensional directional data under rotationally symmetric distributions. Besides, we establish the corresponding theoretical results, including the limiting null distributions, the asymptotic power under the local alternative and the asymptotic relative efficiency results in Section 3. Then, we investigate the numerical performance of the proposed test in comparison with its main competitors in Section 4. Finally, we conclude the paper with some discussions in Section 5, and relegate some additional numerical results as well as the technical proofs to the appendix. 


\section{Test statistics}

Let $\boldsymbol{X}_{1}, \cdots, \boldsymbol{X}_{n} \in \mathcal{S}^{p-1} \doteq\left\{\mathbf{x} \in \mathbb{R}^{p}:\|\mathbf{x}\|=1\right\}$ be a sequence of $p$-dimensional independent and identically distributed (iid) observations from a rotationally symmetric distribution with spherical location $\boldsymbol{\theta} \in \mathcal{S}^{p-1}$. We recall that a random vector $\boldsymbol{X}_{i}$ is said to be rotationally symmetric about some location $\boldsymbol{\theta} \in \mathcal{S}^{p-1}$ if its distribution is invariant under rotations about $\boldsymbol{\theta}$, i.e. if $\boldsymbol{O} \boldsymbol{X}_{i}$ has the same distribution as $\boldsymbol{X}_{i}$ for any orthogonal $p \times p$ matrix $\boldsymbol{O}$ satisfying $\boldsymbol{O} \boldsymbol{\theta}=\boldsymbol{\theta}$. In particular, $\boldsymbol{X}_{i}$ has the density of $c_{f, p} f\left(\mathbf{x}^{\mathrm{T}} \boldsymbol{\theta}\right)$ with $\mathbf{x} \in \mathcal{S}^{p-1}$, where $f:[-1,1] \rightarrow \mathbb{R}^{+}$ is an absolutely continuous function, called the angular function, and $c_{f, p}$ is the standardization constant. For example, the FvML distribution, one of the most popular members of the rotationally symmetric distributions, is obtained by taking the angular function $t \rightarrow \exp (\kappa t)$, where $t \in[-1,1]$ and $\kappa \geq 0$ is the concentration parameter.

Our interest is to test the location hypotheses

$$
H_{0}: \boldsymbol{\theta}=\boldsymbol{\theta}_{0} \quad \text { versus } H_{1}: \boldsymbol{\theta} \neq \boldsymbol{\theta}_{0},
$$

for some given location $\boldsymbol{\theta}_{0} \in \mathcal{S}^{p-1}$. To test (2.1), we study the family of directional weighted spatial sign tests, where the test statistics are constructed as

$$
\begin{aligned}
T_{n}(\omega) \doteq & \frac{2}{n(n-1)} \sum_{i<j} \sum_{i} \omega\left(\left\|\left(\mathbf{I}_{p}-\boldsymbol{\theta}_{0} \boldsymbol{\theta}_{0}^{\mathrm{T}}\right) \boldsymbol{X}_{i}\right\|\right) \omega\left(\left\|\left(\mathbf{I}_{p}-\boldsymbol{\theta}_{0} \boldsymbol{\theta}_{0}^{\mathrm{T}}\right) \boldsymbol{X}_{j}\right\|\right) \\
& \times \mathbf{U}\left\{\left(\mathbf{I}_{p}-\boldsymbol{\theta}_{0} \boldsymbol{\theta}_{0}^{\mathrm{T}}\right) \boldsymbol{X}_{i}\right\}^{\mathrm{T}} \mathbf{U}\left\{\left(\mathbf{I}_{p}-\boldsymbol{\theta}_{0} \boldsymbol{\theta}_{0}^{\mathrm{T}}\right) \boldsymbol{X}_{j}\right\} .
\end{aligned}
$$

Here, $\omega(\cdot)$ is a nonnegative and continuous weight function on $\mathbb{R}^{+}, \boldsymbol{I}_{p}$ denotes the $p \times p$ identity matrix and $\mathbf{U}(\cdot)$ denotes the spatial sign function with $\mathbf{U}(\boldsymbol{a}) \doteq \boldsymbol{a} /\|\boldsymbol{a}\|$ if $\boldsymbol{a} \neq \mathbf{0}$ and $\mathbf{U}(\boldsymbol{a}) \doteq \mathbf{0}$ if $\boldsymbol{a}=\mathbf{0}$. Define

$$
\widehat{\sigma}_{n}^{2}(\omega) \doteq 2 n^{-4} p^{-1} \sum_{i \neq j} \sum_{i} \omega\left(\left\|\left(\mathbf{I}_{p}-\boldsymbol{\theta}_{0} \boldsymbol{\theta}_{0}^{\mathrm{T}}\right) \boldsymbol{X}_{i}\right\|\right)^{2} \omega\left(\left\|\left(\mathbf{I}_{p}-\boldsymbol{\theta}_{0} \boldsymbol{\theta}_{0}^{\mathrm{T}}\right) \boldsymbol{X}_{j}\right\|\right)^{2} .
$$

According to Theorems 3.1 and 3.2 presented in the next section, for each $\omega, H_{0}$ will be rejected when $T_{n}(\omega) / \sqrt{\widehat{\sigma}_{n}^{2}(\omega)}>z_{\alpha}$, where $z_{\alpha}$ is the upper $\alpha$-quantile of the standard normal distribution $\mathcal{N}(0,1)$ with significance level $\alpha$. We will call this test the $T_{n}(\omega)$-based test.

In fact, some members of this family are closely related to existing tests for (2.1). For example, taking $\omega(t)=\omega_{\mathrm{N}}(t) \doteq t$ for $t \geq 0$, i.e. using the norm (N) weight function $\omega_{\mathrm{N}}$, the test statistic is

$$
\begin{aligned}
T_{n}\left(\omega_{\mathrm{N}}\right)= & \frac{2}{n(n-1)} \sum_{i<j}\left\|\left(\mathbf{I}_{p}-\boldsymbol{\theta}_{0} \boldsymbol{\theta}_{0}^{\mathrm{T}}\right) \boldsymbol{X}_{i}\right\|\left\|\left(\mathbf{I}_{p}-\boldsymbol{\theta}_{0} \boldsymbol{\theta}_{0}^{\mathrm{T}}\right) \boldsymbol{X}_{j}\right\| \\
& \times \mathbf{U}\left\{\left(\mathbf{I}_{p}-\boldsymbol{\theta}_{0} \boldsymbol{\theta}_{0}^{\mathrm{T}}\right) \boldsymbol{X}_{i}\right\}^{\mathrm{T}} \mathbf{U}\left\{\left(\mathbf{I}_{p}-\boldsymbol{\theta}_{0} \boldsymbol{\theta}_{0}^{\mathrm{T}}\right) \boldsymbol{X}_{j}\right\} .
\end{aligned}
$$

The main part of $T_{n}\left(\omega_{\mathrm{N}}\right)$ is the same as that of the high-dimensional Watson test statistic proposed by [12]:

$$
\widetilde{W}_{n} \doteq \frac{\sqrt{2(p-1)}}{\sum_{i=1}^{n} v_{i 0}^{2}} \sum_{i<j} \sum_{i}\left\|\left(\mathbf{I}_{p}-\boldsymbol{\theta}_{0} \boldsymbol{\theta}_{0}^{\mathrm{T}}\right) \boldsymbol{X}_{i}\right\|\left\|\left(\mathbf{I}_{p}-\boldsymbol{\theta}_{0} \boldsymbol{\theta}_{0}^{\mathrm{T}}\right) \boldsymbol{X}_{j}\right\|
$$




$$
\times \mathbf{U}\left\{\left(\mathbf{I}_{p}-\boldsymbol{\theta}_{0} \boldsymbol{\theta}_{0}^{\mathrm{T}}\right) \boldsymbol{X}_{i}\right\}^{\mathrm{T}} \mathbf{U}\left\{\left(\mathbf{I}_{p}-\boldsymbol{\theta}_{0} \boldsymbol{\theta}_{0}^{\mathrm{T}}\right) \boldsymbol{X}_{j}\right\},
$$

where $v_{i 0} \doteq\left\|\left(\mathbf{I}_{p}-\boldsymbol{\theta}_{0} \boldsymbol{\theta}_{0}^{\mathrm{T}}\right) \boldsymbol{X}_{i}\right\|$.

The relationship between the two statistics is discussed in the following proposition. To present this relationship, we need to impose the following condition.

$(\mathrm{C} 0) \mathbb{E}\left(v_{i}^{4}\right) / \mathbb{E}^{2}\left(v_{i}^{2}\right)=O(1), \mathbb{E}\left(v_{i}^{2}\right) \neq 0$ and for each $i \in\{1 \cdots, n\}, \mathbb{E}\left\{v_{i}^{-4}\right\}$ exists for sufficiently large $p$.

Here, $v_{i} \doteq\left\|\left(\mathbf{I}_{p}-\boldsymbol{\theta} \boldsymbol{\theta}^{\mathrm{T}}\right) \boldsymbol{X}_{i}\right\|=\left(1-u_{i}^{2}\right)^{1 / 2}$, where $u_{i} \doteq \boldsymbol{X}_{i}^{\mathrm{T}} \boldsymbol{\theta}$ and $\boldsymbol{X}_{i}$ is rotationally symmetric about $\boldsymbol{\theta}$ with concentration parameter $\kappa$. The probability density function of $u_{i}$ is

$$
c_{p, f, \kappa}\left(1-u^{2}\right)^{(p-3) / 2} f_{\kappa}(u),
$$

where $u \in[-1,1]$ and $c_{p, f, \kappa} \doteq 1 / \int_{-1}^{1}\left(1-u^{2}\right)^{(p-3) / 2} f_{\kappa}(u) d u$. It can be seen that the distributions of $u_{i}$ and $v_{i}$ do not depend on $\boldsymbol{\theta}$.

Proposition 2.1. As $n, p \rightarrow \infty$, under condition (CO) and $H_{0}$,

$$
\widetilde{W}_{n} / \frac{T_{n}\left(\omega_{\mathrm{N}}\right)}{\sqrt{\widehat{\sigma}_{n}^{2}\left(\omega_{N}\right)}} \rightarrow 1 \text { in probability. }
$$

This proposition indicates that the $T_{n}\left(\omega_{\mathrm{N}}\right)$-based and $\widetilde{W}_{n}$-based tests are asymptotically equivalent, where the $\widetilde{W}_{n}$-based test rejects $H_{0}$ when $\widetilde{W}_{n}>z_{\alpha}$. We recall that all proofs are discussed in the appendix.

In fact, $\widetilde{W}_{n}$ is a standardized version of the traditional Watson test statistic $[23,20]$

$$
W_{n} \doteq \frac{n(p-1) \overline{\boldsymbol{X}}_{n}^{\mathrm{T}}\left(\boldsymbol{I}_{p}-\boldsymbol{\theta}_{0} \boldsymbol{\theta}_{0}^{\mathrm{T}}\right) \overline{\boldsymbol{X}}_{n}}{1-\frac{1}{n} \sum_{i=1}^{n}\left(\boldsymbol{X}_{i}^{\mathrm{T}} \boldsymbol{\theta}_{0}\right)^{2}},
$$

with asymptotic mean $p-1$ and asymptotic variance $2(p-1)$ under the null hypothesis, where $\overline{\boldsymbol{X}}_{n} \doteq \frac{1}{n} \sum_{i=1}^{n} \boldsymbol{X}_{i}$. The $W_{n}$-based test rejects $H_{0}$ when $W_{n}$ is larger than the upper $\alpha$-quantile of $\chi_{p-1}^{2}$.

Taking $\omega(t)=\omega_{\mathrm{C}}(t) \doteq 1$ for $t \geq 0$, i.e. using the constant (C) weight function $\omega_{\mathrm{C}}$, the test statistic is

$$
\left.T_{n}\left(\omega_{\mathrm{C}}\right)=\frac{2}{n(n-1)} \sum_{i<j} \sum_{\mathbf{U}} \mathbf{U}\left(\mathbf{I}_{p}-\boldsymbol{\theta}_{0} \boldsymbol{\theta}_{0}^{\mathrm{T}}\right) \boldsymbol{X}_{i}\right\}^{\mathrm{T}} \mathbf{U}\left\{\left(\mathbf{I}_{p}-\boldsymbol{\theta}_{0} \boldsymbol{\theta}_{0}^{\mathrm{T}}\right) \boldsymbol{X}_{j}\right\}
$$

which is very similar to the test that was mentioned in [18].

In addition to these existing ones, we also consider some other members of this family. Taking $\omega(t)=\omega_{\mathrm{IN}}(t) \doteq t^{-1}$ for $t \geq 0$, i.e. using the inverse norm (IN) weight function $\omega_{\text {IN }}$, the test statistic is

$$
\begin{aligned}
T_{n}\left(\omega_{\mathrm{IN}}\right)= & \frac{2}{n(n-1)} \sum_{i<j}\left\|\left(\mathbf{I}_{p}-\boldsymbol{\theta}_{0} \boldsymbol{\theta}_{0}^{\mathrm{T}}\right) \boldsymbol{X}_{i}\right\|^{-1}\left\|\left(\mathbf{I}_{p}-\boldsymbol{\theta}_{0} \boldsymbol{\theta}_{0}^{\mathrm{T}}\right) \boldsymbol{X}_{j}\right\|^{-1} \\
& \times \mathbf{U}\left\{\left(\mathbf{I}_{p}-\boldsymbol{\theta}_{0} \boldsymbol{\theta}_{0}^{\mathrm{T}}\right) \boldsymbol{X}_{i}\right\}^{\mathrm{T}} \mathbf{U}\left\{\left(\mathbf{I}_{p}-\boldsymbol{\theta}_{0} \boldsymbol{\theta}_{0}^{\mathrm{T}}\right) \boldsymbol{X}_{j}\right\},
\end{aligned}
$$


which can be regarded as an extension of the inverse norm sign test (INST) proposed by [6] on directional data. In this paper, we name the $T_{n}\left(\omega_{\mathrm{IN}}\right)$-based test as the inverse norm weight spatial sign test.

Taking $\omega(t)=\omega_{\mathrm{S}}(t) \doteq t^{2}$ and $\omega(t)=\omega_{\mathrm{R}}(t) \doteq t^{1 / 2}$ respectively, the test statistics are

$$
\begin{aligned}
T_{n}\left(\omega_{\mathrm{S}}\right)= & \frac{2}{n(n-1)} \sum_{i<j} \sum_{i}\left\|\left(\mathbf{I}_{p}-\boldsymbol{\theta}_{0} \boldsymbol{\theta}_{0}^{\mathrm{T}}\right) \boldsymbol{X}_{i}\right\|^{2}\left\|\left(\mathbf{I}_{p}-\boldsymbol{\theta}_{0} \boldsymbol{\theta}_{0}^{\mathrm{T}}\right) \boldsymbol{X}_{j}\right\|^{2} \\
& \times \mathbf{U}\left\{\left(\mathbf{I}_{p}-\boldsymbol{\theta}_{0} \boldsymbol{\theta}_{0}^{\mathrm{T}}\right) \boldsymbol{X}_{i}\right\}^{\mathrm{T}} \mathbf{U}\left\{\left(\mathbf{I}_{p}-\boldsymbol{\theta}_{0} \boldsymbol{\theta}_{0}^{\mathrm{T}}\right) \boldsymbol{X}_{j}\right\},
\end{aligned}
$$

and

$$
\begin{aligned}
T_{n}\left(\omega_{\mathrm{R}}\right)= & \frac{2}{n(n-1)} \sum_{i<j} \sum_{j}\left\|\left(\mathbf{I}_{p}-\boldsymbol{\theta}_{0} \boldsymbol{\theta}_{0}^{\mathrm{T}}\right) \boldsymbol{X}_{i}\right\|^{1 / 2}\left\|\left(\mathbf{I}_{p}-\boldsymbol{\theta}_{0} \boldsymbol{\theta}_{0}^{\mathrm{T}}\right) \boldsymbol{X}_{j}\right\|^{1 / 2} \\
& \times \mathbf{U}\left\{\left(\mathbf{I}_{p}-\boldsymbol{\theta}_{0} \boldsymbol{\theta}_{0}^{\mathrm{T}}\right) \boldsymbol{X}_{i}\right\}^{\mathrm{T}} \mathbf{U}\left\{\left(\mathbf{I}_{p}-\boldsymbol{\theta}_{0} \boldsymbol{\theta}_{0}^{\mathrm{T}}\right) \boldsymbol{X}_{j}\right\}
\end{aligned}
$$

Now, we have introduced the family of directional weighted spatial sign tests. In the following section, we will establish the theoretical results of the whole family, which imply that the $T_{n}\left(\omega_{\text {IN }}\right)$-based test has the maximum asymptotic power among this family. Then, extensive numerical results will demonstrate the empirical power advantages of the $T_{n}\left(\omega_{\mathrm{IN}}\right)$-based test compared with some other members of this family as well as some existing tests.

\section{Theoretical results}

In this section, we will establish the theoretical results of the whole family of directional weighted spatial sign tests with the general form of weight function $\omega(\cdot)$.

\subsection{Null distribution}

(C1) $b_{4}(\omega)=O\left\{b_{2}(\omega)^{2}\right\}, b_{2}(\omega) \neq 0$ and for each $i \in\{1 \cdots, n\}, \mathbb{E}\left\{v_{i}^{-4}\right\}$ exists for sufficiently large $p$.

Here, $b_{k}(\omega) \doteq \mathbb{E}\left\{\omega^{k}\left(v_{i}\right)\right\}$ for any positive integer $k$. Note that by choosing $\omega=\omega_{\mathrm{N}}$, condition $(\mathrm{C} 1)$ becomes condition $(\mathrm{C} 0)$, which leads to $\mathbb{E}\left(v_{i}^{4}\right) / \mathbb{E}^{2}\left(v_{i}^{2}\right)=$ $o(n)$, previously used in Theorem 3.1 (iv) of [12] and Theorem 3.1 (b) of [20].

Theorem 3.1. Under condition (C1) and $H_{0}$, as $n, p \rightarrow \infty, T_{n}(\omega) / \sigma_{n}(\omega) \rightarrow$ $\mathcal{N}(0,1)$ in distribution, where $\sigma_{n}^{2}(\omega) \doteq 2 n^{-2} p^{-1} b_{2}^{2}(\omega)$.

We find a ratio-consistent estimation of $\sigma_{n}^{2}(\omega)$ in the following theorem.

Theorem 3.2. Under condition (C1) and $H_{0}$, as $n, p \rightarrow \infty, \widehat{\sigma}_{n}^{2}(\omega) / \sigma_{n}^{2}(\omega) \rightarrow 1$ in probability, where $\widehat{\sigma}_{n}^{2}(\omega)$ is defined in $(2.3)$. 


\subsection{Asymptotic power}

Then, we investigate the asymptotic distribution of $T_{n}(\omega)$ under the following local alternative.

$$
\begin{aligned}
& \left\|\boldsymbol{\theta}-\boldsymbol{\theta}_{0}\right\|^{2} \sqrt{\mathbb{E}\left(v_{i}^{-4}\right)}=O\left(n^{-1} p^{-1 / 2}\right) \text { and } \operatorname{var}\left(u_{i}\right)=o(1) \text { for each } i \in \\
& \{1, \cdots, n\}, \text { where } u_{i}=\boldsymbol{X}_{i}^{\mathrm{T}} \boldsymbol{\theta} .
\end{aligned}
$$

Note that $\operatorname{var}\left(u_{i}\right)=o(1)$ is a weaker condition than condition (c), i.e. $\sqrt{p} \mathbb{E}\left(u_{i}^{2}\right)=$ $o(1)$, of Theorem 3.1 in [20] due to $\operatorname{var}\left(u_{i}\right) \leq \mathbb{E}\left(u_{i}^{2}\right)$, which was used to derive the local power of $\widetilde{W}_{n}$. Condition (C2) ensures that the difference between $\boldsymbol{\theta}$ and $\boldsymbol{\theta}_{0}$ is not too large, so that the variance of $T_{n}(\omega)$ can be asymptotically described by $\sigma_{n}^{2}(\omega)$.

Theorem 3.3. Under conditions (C1)-(C2), as $n, p \rightarrow \infty$,

$$
\left[T_{n}(\omega)-c_{0}(\omega)^{2}\left\{\boldsymbol{\theta}^{\mathrm{T}}\left(\boldsymbol{\theta} \boldsymbol{\theta}^{\mathrm{T}}-\boldsymbol{\theta}_{0} \boldsymbol{\theta}_{0}^{\mathrm{T}}\right) \boldsymbol{\theta}\right\}\right] / \sigma_{n}(\omega) \rightarrow \mathcal{N}(0,1) \text { in distribution, }
$$

where $c_{0}(\omega) \doteq \mathbb{E}\left(u_{i}\right) \mathbb{E}\left\{\omega\left(v_{i}\right) v_{i}^{-1}\right\}$.

According to Theorems 3.1 and 3.3, the local power of the $T_{n}(\omega)$-based test against an alternative $\boldsymbol{\theta}$ that satisfies condition (C2) is

$$
\begin{aligned}
& \beta_{n, p}(\omega) \\
\doteq & \mathrm{P}_{\boldsymbol{\theta}}\left[T_{n}(\omega)>\sigma_{n}(\omega) z_{\alpha}\right] \\
= & \mathrm{P}_{\boldsymbol{\theta}}\left[\frac{T_{n}(\omega)-c_{0}(\omega)^{2}\left\{\boldsymbol{\theta}^{\mathrm{T}}\left(\boldsymbol{\theta} \boldsymbol{\theta}^{\mathrm{T}}-\boldsymbol{\theta}_{0} \boldsymbol{\theta}_{0}^{\mathrm{T}}\right) \boldsymbol{\theta}\right\}}{\sigma_{n}(\omega)}>z_{\alpha}-\frac{c_{0}(\omega)^{2}\left\{\boldsymbol{\theta}^{\mathrm{T}}\left(\boldsymbol{\theta} \boldsymbol{\theta}^{\mathrm{T}}-\boldsymbol{\theta}_{0} \boldsymbol{\theta}_{0}^{\mathrm{T}}\right) \boldsymbol{\theta}\right\}}{\sigma_{n}(\omega)}\right] .
\end{aligned}
$$

Then, we obtain the asymptotic power of the $T_{n}(\omega)$-based test:

$$
\begin{aligned}
& \beta(\omega) \\
\doteq & \lim _{n, p \rightarrow \infty} \beta_{n, p}(\omega) \\
= & \lim _{n, p \rightarrow \infty} \Phi\left[-z_{\alpha}+\frac{\mathbb{E}^{2}\left(u_{i}\right) \mathbb{E}^{2}\left\{\omega\left(v_{i}\right) v_{i}^{-1}\right\}}{\mathbb{E}\left\{\omega^{2}\left(v_{i}\right)\right\}} \frac{p^{1 / 2} n\left(\boldsymbol{\theta}^{\mathrm{T}}\left(\boldsymbol{\theta} \boldsymbol{\theta}^{\mathrm{T}}-\boldsymbol{\theta}_{0} \boldsymbol{\theta}_{0}^{\mathrm{T}}\right) \boldsymbol{\theta}\right)}{\sqrt{2}}\right] .
\end{aligned}
$$

To find a weight function $\omega$ that can make $\beta(\omega)$ reach the maximum value, we only need to find the maximum value of $\mathbb{E}^{2}\left(u_{i}\right) \mathbb{E}^{2}\left\{\omega\left(v_{i}\right) v_{i}^{-1}\right\} / \mathbb{E}\left\{\omega^{2}\left(v_{i}\right)\right\}$, because $\Phi\left[-z_{\alpha}+\frac{\mathbb{E}^{2}\left(u_{i}\right) \mathbb{E}^{2}\left\{\omega\left(v_{i}\right) v_{i}^{-1}\right\}}{\mathbb{E}\left\{\omega^{2}\left(v_{i}\right)\right\}} \frac{p^{1 / 2} n\left(\boldsymbol{\theta}^{\mathrm{T}}\left(\boldsymbol{\theta} \boldsymbol{\theta}^{\mathrm{T}}-\boldsymbol{\theta}_{0} \boldsymbol{\theta}_{0}^{\mathrm{T}}\right) \boldsymbol{\theta}\right)}{\sqrt{2}}\right]$ in (3.1) is an increasing function of $\mathbb{E}^{2}\left(u_{i}\right) \mathbb{E}^{2}\left\{\omega\left(v_{i}\right) v_{i}^{-1}\right\} / \mathbb{E}\left\{\omega^{2}\left(v_{i}\right)\right\}$. By proving $\mathbb{E}^{2}\left\{\omega\left(v_{i}\right) v_{i}^{-1}\right\} / \mathbb{E}\left\{\omega^{2}\left(v_{i}\right)\right\} \leq$ $\mathbb{E}\left(v_{i}{ }^{-2}\right)$, we will obtain that taking $\omega=\omega_{\text {IN }}$ makes $\beta(\omega)$ reach the maximum value.

Theorem 3.4. For all the weight functions satisfying conditions (C1)-(C2), as $n, p \rightarrow \infty$, the maximum value of the asymptotic power $\beta(\omega)$ is

$$
\beta\left(\omega_{\mathrm{IN}}\right)=\lim _{n, p \rightarrow \infty} \Phi\left[-z_{\alpha}+\mathbb{E}^{2}\left(u_{i}\right) \mathbb{E}\left(v_{i}{ }^{-2}\right) \frac{p^{1 / 2} n\left\{\boldsymbol{\theta}^{\mathrm{T}}\left(\boldsymbol{\theta} \boldsymbol{\theta}^{\mathrm{T}}-\boldsymbol{\theta}_{0} \boldsymbol{\theta}_{0}^{\mathrm{T}}\right) \boldsymbol{\theta}\right\}}{\sqrt{2}}\right] .
$$


In contrast, the asymptotic power of the $T_{n}\left(\omega_{\mathrm{C}}\right)$-based, $T_{n}\left(\omega_{\mathrm{N}}\right)$-based, $T_{n}\left(\omega_{\mathrm{S}}\right)$-based and $T_{n}\left(\omega_{\mathrm{R}}\right)$-based tests are

$$
\begin{aligned}
& \beta\left(\omega_{\mathrm{N}}\right)=\lim _{n, p \rightarrow \infty} \Phi\left[-z_{\alpha}+\mathbb{E}^{2}\left(u_{i}\right) \mathbb{E}^{-1}\left(v_{i}^{2}\right) \frac{p^{1 / 2} n\left\{\boldsymbol{\theta}^{\mathrm{T}}\left(\boldsymbol{\theta} \boldsymbol{\theta}^{\mathrm{T}}-\boldsymbol{\theta}_{0} \boldsymbol{\theta}_{0}^{\mathrm{T}}\right) \boldsymbol{\theta}\right\}}{\sqrt{2}}\right], \\
& \beta\left(\omega_{\mathrm{C}}\right)=\lim _{n, p \rightarrow \infty} \Phi\left[-z_{\alpha}+\mathbb{E}^{2}\left(u_{i}\right) \mathbb{E}^{2}\left(v_{i}^{-1}\right) \frac{p^{1 / 2} n\left\{\boldsymbol{\theta}^{\mathrm{T}}\left(\boldsymbol{\theta} \boldsymbol{\theta}^{\mathrm{T}}-\boldsymbol{\theta}_{0} \boldsymbol{\theta}_{0}^{\mathrm{T}}\right) \boldsymbol{\theta}\right\}}{\sqrt{2}}\right], \\
& \beta\left(\omega_{\mathrm{S}}\right)=\lim _{n, p \rightarrow \infty} \Phi\left[-z_{\alpha}+\mathbb{E}^{2}\left(u_{i}\right) \frac{\mathbb{E}^{2}\left(v_{i}\right)}{\mathbb{E}\left(v_{i}^{4}\right)} \frac{p^{1 / 2} n\left\{\boldsymbol{\theta}^{\mathrm{T}}\left(\boldsymbol{\theta} \boldsymbol{\theta}^{\mathrm{T}}-\boldsymbol{\theta}_{0} \boldsymbol{\theta}_{0}^{\mathrm{T}}\right) \boldsymbol{\theta}\right\}}{\sqrt{2}}\right], \\
& \beta\left(\omega_{\mathrm{R}}\right)=\lim _{n, p \rightarrow \infty} \Phi\left[-z_{\alpha}+\mathbb{E}^{2}\left(u_{i}\right) \frac{\mathbb{E}^{2}\left(v_{i}^{-1 / 2}\right)}{\mathbb{E}\left(v_{i}\right)} \frac{p^{1 / 2} n\left\{\boldsymbol{\theta}^{\mathrm{T}}\left(\boldsymbol{\theta} \boldsymbol{\theta}^{\mathrm{T}}-\boldsymbol{\theta}_{0} \boldsymbol{\theta}_{0}^{\mathrm{T}}\right) \boldsymbol{\theta}\right\}}{\sqrt{2}}\right],
\end{aligned}
$$

respectively.

Next, we consider the asymptotic power of the $T_{n}(\omega)$-based test in situation of the FvML distribution.

Corollary 3.1. Suppose $\boldsymbol{X}_{i}$ follows a FvML distribution. Under conditions (C1) and (C2), as $n, p \rightarrow \infty$, the asymptotic power of the $T_{n}(\omega)$-based test is

$$
\beta=\lim _{n, p \rightarrow \infty} \Phi\left[-z_{\alpha}+\mathbb{E}^{2}\left(u_{i}\right) \mathbb{E}\left(v_{i}^{-2}\right) \frac{p^{1 / 2} n\left\{\boldsymbol{\theta}^{\mathrm{T}}\left(\boldsymbol{\theta} \boldsymbol{\theta}^{\mathrm{T}}-\boldsymbol{\theta}_{0} \boldsymbol{\theta}_{0}^{\mathrm{T}}\right) \boldsymbol{\theta}\right\}}{\sqrt{2}}\right],
$$

which does not depend on the choice of the weight function $\omega$.

This corollary indicates that if $\boldsymbol{X}_{i}$ follows a FvML distribution, all the directional weighted spatial sign tests have the same asymptotic power.

\subsection{Asymptotic relative efficiency}

Then, we derive the asymptotic relative efficiencies (AREs) between the $T_{n}\left(\omega_{\text {IN }}\right)$ based test and the $T_{n}\left(\omega_{\mathrm{C}}\right)$-based, $T_{n}\left(\omega_{\mathrm{N}}\right)$-based, $T_{n}\left(\omega_{\mathrm{S}}\right)$-based, $T_{n}\left(\omega_{\mathrm{R}}\right)$-based tests, respectively. Specifically,

$$
\begin{aligned}
& \mathrm{ARE}_{\mathrm{IN}, \mathrm{N}}=\mathbb{E}\left(v_{i}^{-2}\right) \mathbb{E}\left(v_{i}^{2}\right) \geq 1, \\
& \mathrm{ARE}_{\mathrm{IN}, \mathrm{C}}=\mathbb{E}\left(v_{i}^{-2}\right) \mathbb{E}^{-2}\left(v_{i}^{-1}\right)=1+\operatorname{var}\left(v_{i}^{-1}\right) \mathbb{E}^{-2}\left(v_{i}^{-1}\right) \geq 1, \\
& \mathrm{ARE}_{\mathrm{IN}, \mathrm{S}}=\mathbb{E}\left(v_{i}^{-2}\right) \mathbb{E}\left(v_{i}^{4}\right) \mathbb{E}^{-2}\left(v_{i}\right) \geq 1, \\
& \mathrm{ARE}_{\mathrm{IN}, \mathrm{R}}=\mathbb{E}\left(v_{i}^{-2}\right) \mathbb{E}\left(v_{i}\right) \mathbb{E}^{-2}\left(v_{i}^{-1 / 2}\right) \geq 1 .
\end{aligned}
$$

For rotationally symmetric distributions, there are many choices of angular functions. Below, we present the formulas for calculating the AREs under some commonly used angular functions. First, when $\boldsymbol{X}_{i}$ follows a rotationally symmetric distribution, the density function of $\boldsymbol{X}_{i}$ is $c_{p, f, \kappa} f_{\kappa}\left(\mathbf{x}^{\mathrm{T}} \theta\right)$, where $c_{p, f, \kappa}=1 / \int_{-1}^{1}\left(1-t^{2}\right)^{(p-3) / 2} f_{\kappa}(t) d t$. Hence,

$$
\operatorname{ARE}_{\mathrm{IN}, \mathrm{N}}=\frac{c_{p, f, \kappa}^{2}}{c_{p-2, f, \kappa} c_{p+2, f, \kappa}},
$$




$$
\begin{aligned}
\mathrm{ARE}_{\mathrm{IN}, \mathrm{C}} & =\frac{c_{p-1, f, \kappa}^{2}}{c_{p-2, f, \kappa} c_{p, f, \kappa}}, \\
\mathrm{ARE}_{\mathrm{IN}, \mathrm{S}} & =\frac{c_{p+1, f, \kappa}^{2}}{c_{p-2, f, \kappa} c_{p+4, f, \kappa}}, \\
\mathrm{ARE}_{\mathrm{IN}, \mathrm{R}} & =\frac{c_{p-\frac{1}{2}, f, \kappa}^{2}}{c_{p-2, f, \kappa_{1}} c_{p+1, f, \kappa_{1}}} .
\end{aligned}
$$

Next, when $\boldsymbol{X}_{i}$ follows the mixture of two rotationally symmetric distributions with the same location parameter, the density function of $\boldsymbol{X}_{i}$ can be denoted as

$$
\lambda c_{p, f, \kappa_{1}} f_{\kappa_{1}}\left(\mathbf{x}^{\mathrm{T}} \boldsymbol{\theta}\right)+(1-\lambda) c_{p, f, \kappa_{2}} f_{\kappa_{2}}\left(\mathbf{x}^{\mathrm{T}} \boldsymbol{\theta}\right),
$$

where $c_{p, f, \kappa}=1 / \int_{-1}^{1}\left(1-t^{2}\right)^{(p-3) / 2} f_{\kappa}(t) d t$. Then, we have

$$
\begin{aligned}
\mathrm{ARE}_{\mathrm{IN}, \mathrm{N}}= & {\left[\lambda \frac{c_{p, f, \kappa_{1}}}{c_{p-2, f, \kappa_{1}}}+(1-\lambda) \frac{c_{p, f, \kappa_{2}}}{c_{p-2, f, \kappa_{2}}}\right] \times\left[\lambda \frac{c_{p, f, \kappa_{1}}}{c_{p+2, f, \kappa_{1}}}+(1-\lambda) \frac{c_{p, f, \kappa_{2}}}{c_{p+2, f, \kappa_{2}}}\right], } \\
\mathrm{ARE}_{\mathrm{IN}, \mathrm{C}}= & {\left[\lambda \frac{c_{p, f, \kappa_{1}}}{c_{p-2, f, \kappa_{1}}}+(1-\lambda) \frac{c_{p, f, \kappa_{2}}}{c_{p-2, f, \kappa_{2}}}\right] /\left[\lambda \frac{c_{p, f, \kappa_{1}}}{c_{p-1, f, \kappa_{1}}}+(1-\lambda) \frac{c_{p, f, \kappa_{2}}}{c_{p-1, f, \kappa_{2}}}\right]^{2}, } \\
\mathrm{ARE}_{\mathrm{IN}, \mathrm{S}}= & {\left[\lambda \frac{c_{p, f, \kappa_{1}}}{c_{p-2, f, \kappa_{1}}}+(1-\lambda) \frac{c_{p, f, \kappa_{2}}}{c_{p-2, f, \kappa_{2}}}\right] \times\left[\lambda \frac{c_{p, f, \kappa_{1}}}{c_{p+4, f, \kappa_{1}}}+(1-\lambda) \frac{c_{p, f, \kappa_{2}}}{c_{p+4, f, \kappa_{2}}}\right] } \\
& /\left[\lambda \frac{c_{p, f, \kappa_{1}}}{c_{p+1, f, \kappa_{1}}}+(1-\lambda) \frac{c_{p, f, \kappa_{2}}}{c_{p+1, f, \kappa_{2}}}\right]^{2}, \\
\mathrm{ARE}_{\mathrm{IN}, \mathrm{R}}= & {\left[\lambda \frac{c_{p, f, \kappa_{1}}}{c_{p-2, f, \kappa_{1}}}+(1-\lambda) \frac{c_{p, f, \kappa_{2}}}{c_{p-2, f, \kappa_{2}}}\right] \times\left[\lambda \frac{c_{p, f, \kappa_{1}}}{c_{p+1, f, \kappa_{1}}}+(1-\lambda) \frac{c_{p, f, \kappa_{2}}}{c_{p+1, f, \kappa_{2}}}\right] } \\
& /\left[\lambda \frac{c_{p, f, \kappa_{1}}}{c_{p-\frac{1}{2}, f, \kappa_{1}}}+(1-\lambda) \frac{c_{p, f, \kappa_{2}}}{c_{p-\frac{1}{2}, f, \kappa_{2}}}\right]^{2} \cdot
\end{aligned}
$$

In the following, we investigate the ARE results numerically under the nonmixed and mixed rotationally symmetric distributions respectively. Here, we consider the following three types of angular functions.

(F1) The FvML angular function $\exp (\kappa t)$ with $t \in[-1,1]$.

(F2) The angular function $4^{-\kappa \cdot \arccos (t)}$ with $t \in[-1,1]$.

(F3) The angular function $6^{-\kappa \cdot \arcsin (t)}$ with $t \in[-1,1]$.

Table 1 presents the corresponding ARE results of the non-mixed rotationally symmetric distributions, where some different choices of $\kappa$ and $p$ are considered. Then, Tables 2, 3 and 4 present the corresponding ARE results of the mixed distributions for (F1)-(F3) respectively, where two settings are considered: (1) $\lambda=0.9$ with $\kappa_{1}=\kappa / 10$ and $\kappa_{2}=10 \kappa ;(2) \lambda=0.6$ with $\kappa_{1}=\kappa / 5$ and $\kappa_{2}=5 \kappa$. Although the $T_{n}\left(\omega_{\mathrm{IN}}\right)$-based test does not show obvious advantages in Table 1 , it has obvious advantages as shown in Tables 2, 3 and 4, which indicates that the proposed $T_{n}\left(\omega_{\mathrm{IN}}\right)$-based test performs better than the other four directional weighted spatial sign tests in situation of mixed distributions. 
TABLE 1

The ARE results of the non-mixed rotationally symmetric distributions for the three types of angular functions.

\begin{tabular}{|c|c|c|c|c|c|}
\hline$p$ & $\kappa$ & $\mathrm{ARE}_{\mathrm{IN}, \mathrm{N}}$ & $\mathrm{ARE}_{\mathrm{IN}, \mathrm{C}}$ & $\mathrm{ARE}_{\mathrm{IN}, \mathrm{S}}$ & $\mathrm{ARE}_{\mathrm{IN}, \mathrm{R}}$ \\
\hline \multicolumn{6}{|c|}{$(\mathrm{F} 1)$} \\
\hline 100 & $p$ & 1.011 & 1.003 & 1.026 & 1.006 \\
\hline 200 & $p$ & 1.006 & 1.001 & 1.013 & 1.003 \\
\hline 300 & $p$ & 1.004 & 1.001 & 1.008 & 1.002 \\
\hline 400 & $p$ & 1.003 & 1.001 & 1.006 & 1.002 \\
\hline 100 & $p^{2}$ & 1.021 & 1.005 & 1.046 & 1.012 \\
\hline 200 & $p^{2}$ & 1.010 & 1.003 & 1.023 & 1.006 \\
\hline 300 & $p^{2}$ & 1.007 & 1.002 & 1.015 & 1.004 \\
\hline 400 & $p^{2}$ & 1.005 & 1.001 & 1.011 & 1.003 \\
\hline \multicolumn{6}{|c|}{$(\mathrm{F} 2)$} \\
\hline 100 & $2 p$ & 1.037 & 1.009 & 1.083 & 1.021 \\
\hline 150 & $2 p$ & 1.024 & 1.006 & 1.055 & 1.014 \\
\hline 200 & $2 p$ & 1.018 & 1.005 & 1.041 & 1.010 \\
\hline 250 & $2 p$ & 1.014 & 1.004 & 1.032 & 1.008 \\
\hline 300 & $2 p$ & 1.012 & 1.003 & 1.027 & 1.007 \\
\hline 100 & $3 p$ & 1.039 & 1.010 & 1.089 & 1.022 \\
\hline 150 & $3 p$ & 1.026 & 1.006 & 1.059 & 1.014 \\
\hline 200 & $3 p$ & 1.019 & 1.005 & 1.044 & 1.011 \\
\hline 250 & $3 p$ & 1.015 & 1.004 & 1.035 & 1.009 \\
\hline 300 & $3 p$ & 1.013 & 1.003 & 1.029 & 1.007 \\
\hline \multicolumn{6}{|c|}{ (F3) } \\
\hline 100 & $2 p$ & 1.038 & 1.010 & 1.088 & 1.022 \\
\hline 150 & $2 p$ & 1.025 & 1.006 & 1.057 & 1.014 \\
\hline 200 & $2 p$ & 1.019 & 1.005 & 1.043 & 1.011 \\
\hline 250 & $2 p$ & 1.015 & 1.004 & 1.034 & 1.008 \\
\hline 300 & $2 p$ & 1.013 & 1.003 & 1.028 & 1.007 \\
\hline 100 & $3 p$ & 1.040 & 1.010 & 1.091 & 1.022 \\
\hline 150 & $3 p$ & 1.026 & 1.007 & 1.060 & 1.015 \\
\hline 200 & $3 p$ & 1.020 & 1.005 & 1.045 & 1.011 \\
\hline 250 & $3 p$ & 1.016 & 1.004 & 1.035 & 1.009 \\
\hline 300 & $3 p$ & 1.013 & 1.003 & 1.029 & 1.007 \\
\hline
\end{tabular}

\subsection{Two rank-based tests}

To make a more extensive comparison in the following simulation studies, in this subsection we consider two rank-based weights mentioned in [16]. The statistics are

$$
R_{W}=\frac{2}{n(n-1)} \sum_{i<j} R_{i} R_{j} \mathbf{U}\left\{\left(\mathbf{I}_{p}-\boldsymbol{\theta}_{0} \boldsymbol{\theta}_{0}^{\mathrm{T}}\right) \boldsymbol{X}_{i}\right\}^{\mathrm{T}} \mathbf{U}\left\{\left(\mathbf{I}_{p}-\boldsymbol{\theta}_{0} \boldsymbol{\theta}_{0}^{\mathrm{T}}\right) \boldsymbol{X}_{j}\right\}
$$

and

$$
\left.R_{S}=\frac{2}{n(n-1)} \sum_{i<j} \sum_{i} R_{j}^{2} R_{j}^{2} \mathbf{U}\left(\mathbf{I}_{p}-\boldsymbol{\theta}_{0} \boldsymbol{\theta}_{0}^{\mathrm{T}}\right) \boldsymbol{X}_{i}\right\}^{\mathrm{T}} \mathbf{U}\left\{\left(\mathbf{I}_{p}-\boldsymbol{\theta}_{0} \boldsymbol{\theta}_{0}^{\mathrm{T}}\right) \boldsymbol{X}_{j}\right\}
$$

respectively. Here, $R_{i}$ is the rank of $v_{i}$ among $\left\{v_{1}, v_{2}, \cdots, v_{n}\right\}$, 
TABLE 2

The ARE results of the mixed rotationally symmetric distributions for angular function (F1) with two different choices of $\lambda$.

\begin{tabular}{ccccccc}
\hline \hline$\lambda$ & $p$ & $\kappa$ & ARE $_{\mathrm{IN}, \mathrm{N}}$ & ARE $_{\mathrm{IN}, \mathrm{C}}$ & ARE $_{\mathrm{IN}, \mathrm{S}}$ & $\mathrm{ARE}_{\mathrm{IN}, \mathrm{R}}$ \\
\hline 0.9 & 100 & $p^{2}$ & 9.583 & 2.978 & 11.831 & 6.531 \\
& 200 & $p^{2}$ & 9.699 & 2.998 & 11.840 & 6.616 \\
& 300 & $p^{2}$ & 9.739 & 3.005 & 11.842 & 6.645 \\
& 400 & $p^{2}$ & 9.759 & 3.009 & 11.843 & 6.660 \\
& 100 & $p$ & 1.784 & 1.307 & 2.041 & 1.565 \\
& 200 & $p$ & 1.775 & 1.303 & 2.029 & 1.557 \\
& 300 & $p$ & 1.772 & 1.301 & 2.025 & 1.555 \\
& 400 & $p$ & 1.770 & 1.300 & 2.023 & 1.554 \\
0.6 & 100 & $p^{2}$ & 6.520 & 1.570 & 14.042 & 3.220 \\
& 200 & $p^{2}$ & 6.524 & 1.569 & 13.905 & 3.223 \\
& 300 & $p^{2}$ & 6.526 & 1.569 & 13.859 & 3.225 \\
& 400 & $p^{2}$ & 6.527 & 1.568 & 13.837 & 3.225 \\
& 100 & $p$ & 1.869 & 1.184 & 2.851 & 1.463 \\
& 200 & $p$ & 1.855 & 1.180 & 2.825 & 1.455 \\
& 300 & $p$ & 1.851 & 1.179 & 2.817 & 1.452 \\
400 & $p$ & 1.849 & 1.178 & 2.813 & 1.451 \\
\hline \hline
\end{tabular}

TABLE 3

The ARE results of the mixed rotationally symmetric distributions for angular function (F2) with two different choices of $\lambda$.

\begin{tabular}{ccccccc}
\hline \hline$\lambda$ & $p$ & $\kappa$ & ARE $_{\text {IN, } \mathrm{N}}$ & ARE $_{\text {IN,C }}$ & ARE $_{\text {IN }, \mathrm{S}}$ & ARE $_{\text {IN }, \mathrm{R}}$ \\
\hline 0.9 & 100 & $2 p$ & 67.816 & 5.764 & 83.333 & 33.717 \\
& 150 & $2 p$ & 66.906 & 5.731 & 82.109 & 33.328 \\
& 200 & $2 p$ & 66.459 & 5.715 & 81.509 & 33.136 \\
& 250 & $2 p$ & 66.194 & 5.706 & 81.152 & 33.022 \\
& 300 & $2 p$ & 66.018 & 5.699 & 80.916 & 32.947 \\
& 100 & $3 p$ & 139.115 & 6.703 & 172.029 & 59.975 \\
& 150 & $3 p$ & 137.236 & 6.669 & 169.279 & 59.306 \\
& 200 & $3 p$ & 136.313 & 6.653 & 167.930 & 58.977 \\
& 250 & $3 p$ & 135.765 & 6.643 & 167.129 & 58.781 \\
& 300 & $3 p$ & 135.401 & 6.636 & 166.599 & 58.651 \\
0.6 & 100 & $2 p$ & 37.454 & 2.026 & 94.224 & 9.777 \\
& 150 & $2 p$ & 36.951 & 2.018 & 92.583 & 9.682 \\
& 200 & $2 p$ & 36.705 & 2.013 & 91.778 & 9.635 \\
& 250 & $2 p$ & 36.558 & 2.011 & 91.300 & 9.607 \\
& 300 & $2 p$ & 36.461 & 2.009 & 90.984 & 9.589 \\
& 100 & $3 p$ & 64.558 & 2.127 & 168.273 & 13.679 \\
& 150 & $3 p$ & 63.686 & 2.118 & 164.869 & 13.552 \\
& 200 & $3 p$ & 63.257 & 2.114 & 163.200 & 13.489 \\
& 250 & $3 p$ & 63.003 & 2.112 & 162.209 & 13.452 \\
300 & $3 p$ & 62.834 & 2.110 & 161.552 & 13.428 \\
\hline \hline
\end{tabular}

$$
\begin{aligned}
\sigma_{n}^{2}\left(R_{W}\right) & \doteq 2 n^{-4} p^{-1} \sum_{i \neq j} \sum_{i \neq} i^{2}=2 n^{-4} p^{-1}\left\{\left(\sum_{i} i^{2}\right)^{2}-\sum_{i} i^{4}\right\} \\
& =\frac{\left(n^{2}-1\right)\left(4 n^{2}-1\right)(5 n+6)}{90 n^{3} p}
\end{aligned}
$$


TABLE 4

The ARE results of the mixed rotationally symmetric distributions for angular function (F3) with two different choices of $\lambda$.

\begin{tabular}{ccccccc}
\hline \hline$\lambda$ & $p$ & $\kappa$ & ARE $_{\mathrm{IN}, \mathrm{N}}$ & ARE $_{\mathrm{IN}, \mathrm{C}}$ & $\mathrm{ARE}_{\mathrm{IN}, \mathrm{S}}$ & ARE $_{\mathrm{IN}, \mathrm{R}}$ \\
\hline 0.9 & 100 & $2 p$ & 107.571 & 6.380 & 132.694 & 48.888 \\
& 150 & $2 p$ & 106.121 & 6.347 & 130.647 & 48.334 \\
& 200 & $2 p$ & 105.409 & 6.330 & 129.643 & 48.062 \\
& 250 & $2 p$ & 104.985 & 6.321 & 129.047 & 47.900 \\
& 300 & $2 p$ & 104.705 & 6.314 & 128.653 & 47.792 \\
& 100 & $3 p$ & 211.010 & 7.188 & 262.208 & 83.162 \\
150 & $3 p$ & 208.153 & 7.155 & 257.689 & 82.265 \\
200 & $3 p$ & 206.750 & 7.138 & 255.473 & 81.824 \\
250 & $3 p$ & 205.917 & 7.128 & 254.156 & 81.561 \\
& 300 & $3 p$ & 205.364 & 7.122 & 253.284 & 81.387 \\
0.6 & 100 & $2 p$ & 53.704 & 2.095 & 138.357 & 12.217 \\
150 & $2 p$ & 52.980 & 2.086 & 135.713 & 12.102 \\
& 200 & $2 p$ & 52.624 & 2.082 & 134.417 & 12.045 \\
250 & $2 p$ & 52.413 & 2.080 & 133.647 & 12.011 \\
300 & $2 p$ & 52.273 & 2.078 & 133.137 & 11.989 \\
100 & $3 p$ & 84.398 & 2.170 & 223.751 & 16.106 \\
150 & $3 p$ & 83.256 & 2.161 & 218.766 & 15.963 \\
200 & $3 p$ & 82.695 & 2.157 & 216.324 & 15.892 \\
250 & $3 p$ & 82.361 & 2.155 & 214.874 & 15.850 \\
300 & $3 p$ & 82.140 & 2.153 & 213.914 & 15.822 \\
\hline \hline
\end{tabular}

and

$$
\begin{aligned}
\sigma_{n}^{2}\left(R_{S}\right) & \doteq 2 n^{-4} p^{-1} \sum_{i \neq j} \sum_{i} i^{4} j^{4}=2 n^{-4} p^{-1}\left\{\left(\sum_{i} i^{4}\right)^{2}-\sum_{i} i^{8}\right\} \\
& =\frac{\left(n^{2}-1\right)\left(4 n^{2}-1\right)\left(9 n^{5}+20 n^{4}-15 n^{3}-50 n^{2}+n+30\right)}{450 n^{3} p} .
\end{aligned}
$$

Proposition 3.1. Under $H_{0}$, both $R_{W} / \sqrt{\sigma_{n}^{2}\left(R_{W}\right)}$ and $R_{S} / \sqrt{\sigma_{n}^{2}\left(R_{S}\right)}$ are asymptotically standard normal.

The $R_{W}$-based and $R_{S}$-based tests rejects $H_{0}$ when $R_{W} / \sqrt{\sigma_{n}^{2}\left(R_{W}\right)}>z_{\alpha}$ and $R_{S} / \sqrt{\sigma_{n}^{2}\left(R_{S}\right)}>z_{\alpha}$, respectively.

\section{Simulation results}

In this section, we present some simulation results to investigate the performance of the $W_{n}$-based, $\widetilde{W}_{n}$-based, $R_{W}$-based and $R_{S}$-based, $T_{n}\left(\omega_{\mathrm{N}}\right)$-based, $T_{n}\left(\omega_{\mathrm{C}}\right)$ based, $T_{n}\left(\omega_{\mathrm{IN}}\right)$-based, $T_{n}\left(\omega_{\mathrm{S}}\right)$-based and $T_{n}\left(\omega_{\mathrm{R}}\right)$-based tests, abbreviated as $W_{n}$, $\widetilde{W}_{n}, R_{W}, R_{S}, T_{\mathrm{N}}, T_{\mathrm{C}}, T_{\mathrm{IN}}, T_{\mathrm{S}}$ and $T_{\mathrm{R}}$ respectively. We let $\boldsymbol{\theta}_{0}=(1,0, \cdots, 0)^{\mathrm{T}} \in$ $\mathcal{S}^{p-1}$,

$$
\boldsymbol{\theta}=\boldsymbol{\theta}_{0}+\delta\left(-2 \delta, 2 \sqrt{1-\delta^{2}}, 0, \cdots, 0\right)^{\mathrm{T}} \in \mathcal{S}^{p-1}
$$



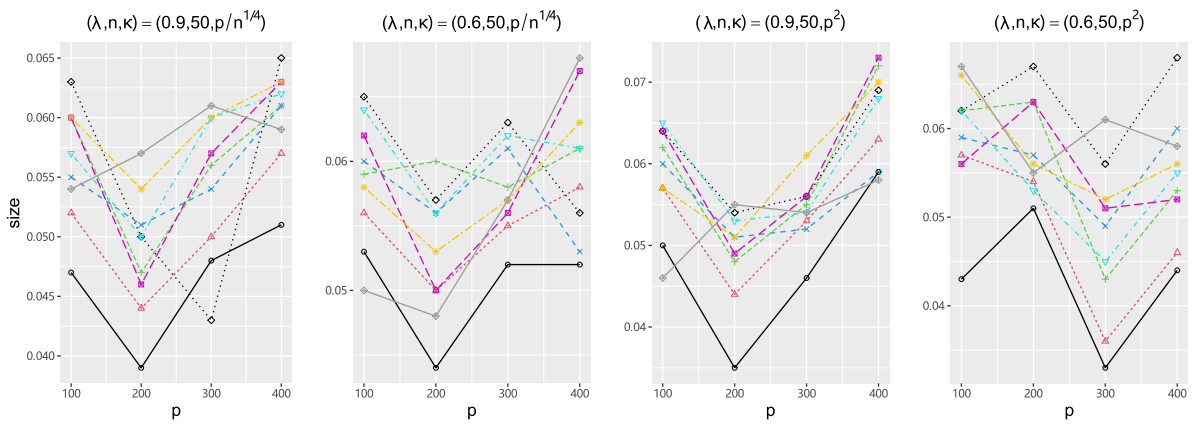

$\rightarrow \mathrm{W}_{\mathrm{n}}^{-+} \mathrm{T}_{\mathrm{N}} \cdots \mathrm{T}_{\mathrm{N}} \rightarrow \mathrm{T}_{\mathrm{S}} \rightarrow \mathrm{R}_{\mathrm{S}}$

$\cdots \widetilde{W}_{n}^{-\kappa-} T_{C} \cdots-T_{R}-R_{W}$

FIG 1. The empirical size results of the nine tests under the mixed rotationally symmetric distributions at $5 \%$ level with angular function (F1) under settings I and II.
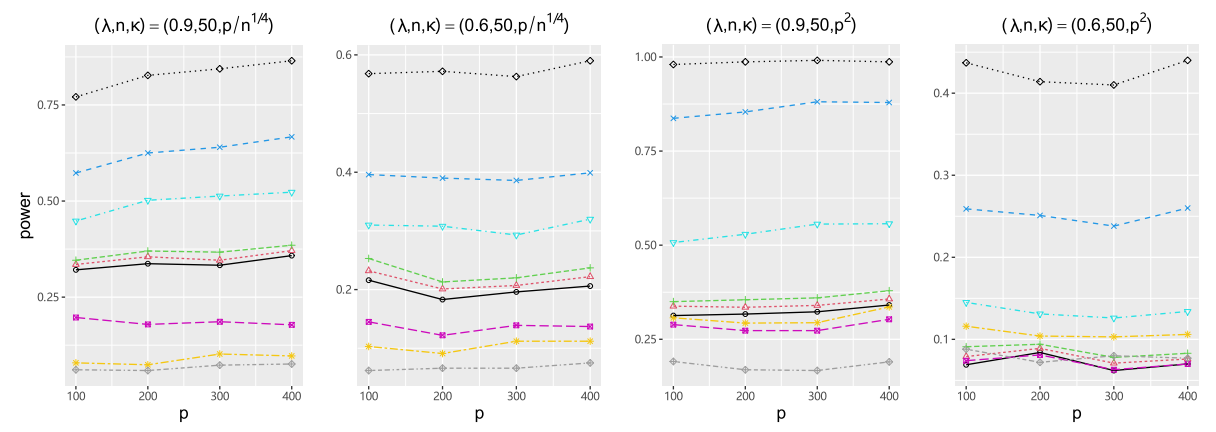

$\rightarrow W_{n}-T_{N} \cdots T_{\text {IN }} \rightarrow T_{s} \rightarrow R_{s}$

$\therefore \widetilde{W}_{n}-*-T_{C} \rightarrow T_{R} \rightarrow R_{W}$

FIG 2. The empirical power results of the nine tests under the mixed rotationally symmetric distributions at $5 \%$ level with angular function (F1) under settings I and II.

We consider two settings of mixed distributions in (3.2), where for setting I, $\left(\lambda, \kappa_{1}, \kappa_{2}, \delta\right)=(0.9, \kappa / 10,10 \kappa, 2 \Delta)$, and for setting II, $\left(\lambda, \kappa_{1}, \kappa_{2}, \delta\right)=(0.6, \kappa / 5$, $5 \kappa, \Delta / 2) . \kappa$ and $\Delta$ will be set according to the angular function type in the following text.

For angular function (F1), we let $n=50, p \in\{100,200,300\}$ and consider two cases:

(1) $\kappa=p / n^{1 / 4}, \Delta=p^{3 / 4} /(\sqrt{n} \kappa)$,

(2) $\kappa=p^{2}, \Delta=p^{1 / 4} / \sqrt{n \kappa}$.

Figures 1 and 2 summarize the empirical size and power results of the nine tests by using angular function (F1), where all the simulation results are based on 1,000 replications. The results in Figure 1 suggest that all these nine tests can 

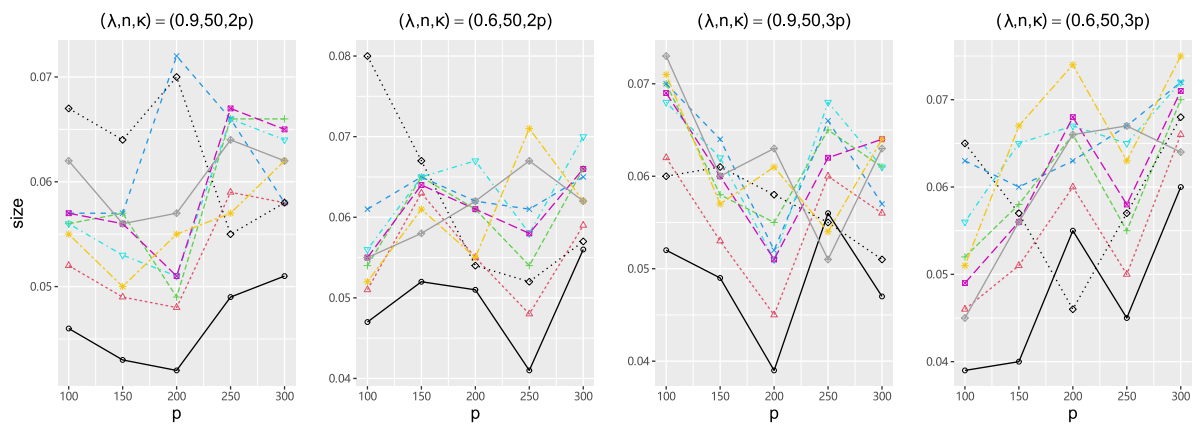

$$
\begin{aligned}
& \rightarrow W_{n}+T_{N} \cdots T_{I N} \rightarrow T_{S} \rightarrow R_{S} \\
& \cdots W_{n^{-*}}-T_{C} \cdots-T_{R} \rightarrow R_{W}
\end{aligned}
$$

FIG 3. The empirical size results of the nine tests under the mixed rotationally symmetric distributions at $5 \%$ level with angular function (F2) under settings I and II.
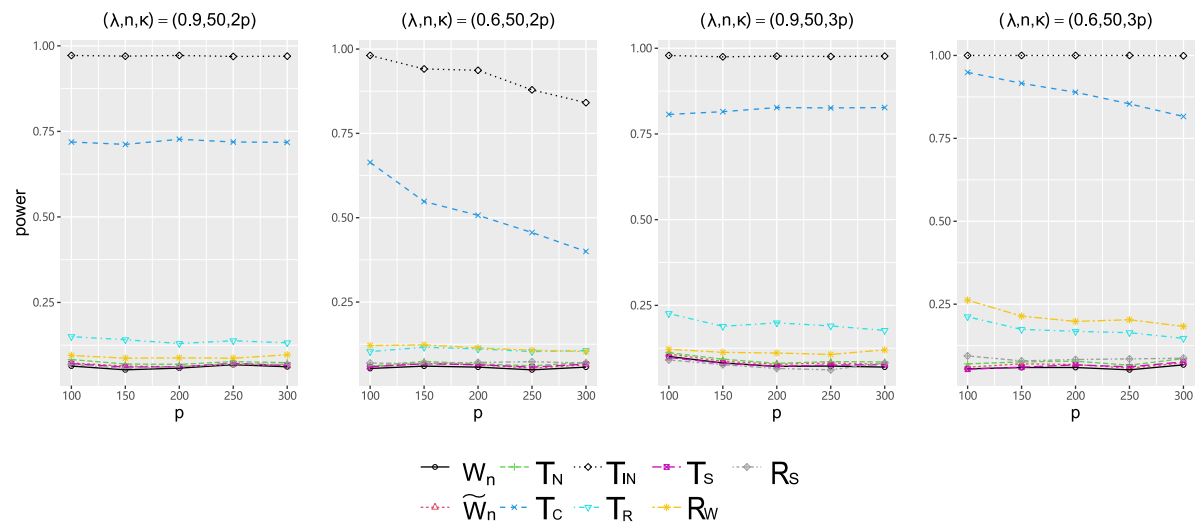

FIG 4. The empirical power results of the nine tests under the mixed rotationally symmetric distributions at 5\% level with angular function (F2) under settings I and II.

properly control the empirical size. For power performance, the power results in Figure 2 suggest that $T_{\mathrm{IN}}$ can outperform the remaining tests.

For angular functions (F2) and (F3), we let $\kappa \in\{2 p, 3 p\}, \Delta=1 /\left(n^{1 / 2} p^{1 / 2}\right)$, $n=50$ and $p \in\{100,150,200,250,300\}$. Figures 3 and 5 summarize the empirical size of the nine tests by using angular functions (F2) and (F3), respectively. Similarly, all the simulation results are based on 1,000 replications. The size results in Figures 3 and 5 are similar to that for angular function (F1). For power performance, Figure 4 presents the power curves for angular function (F2), while Figure 6 presents the power curves for angular function (F3). From Figures 4 and 6 , we see that $T_{\mathrm{IN}}$ and $T_{\mathrm{C}}$ can outperform the remaining tests in power comparison, while $T_{\mathrm{IN}}$ performs much better than $T_{\mathrm{C}}$. In addition, we place some additional simulation results for the non-mixed distributions in Appendix. 

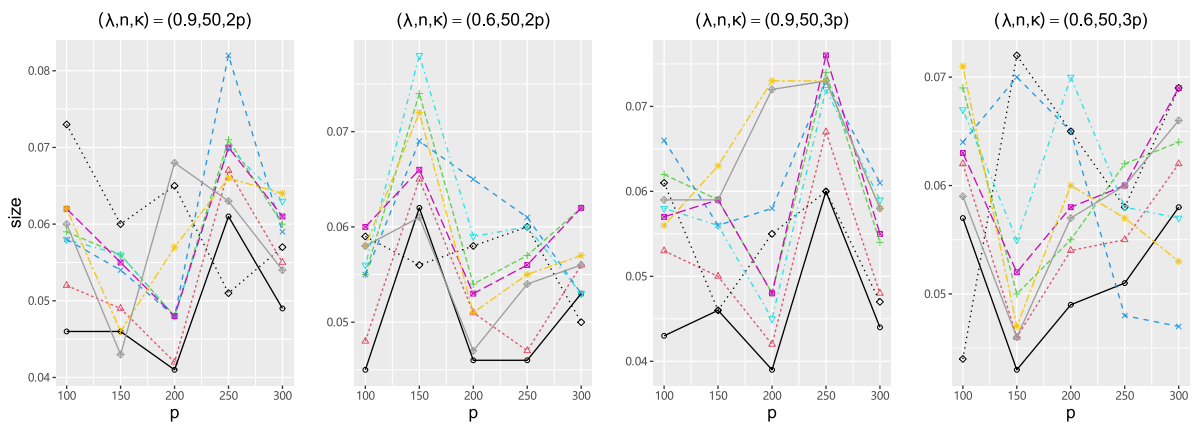

$\rightarrow W_{n}-+T_{N} \cdots \cdot \cdot T_{\text {IN }}-r \cdot T_{S} \rightarrow R_{S}$

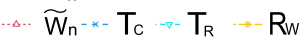

FIG 5. The empirical size results of the nine tests under the mixed rotationally symmetric distributions at $5 \%$ level with angular function (F3) under settings I and II.
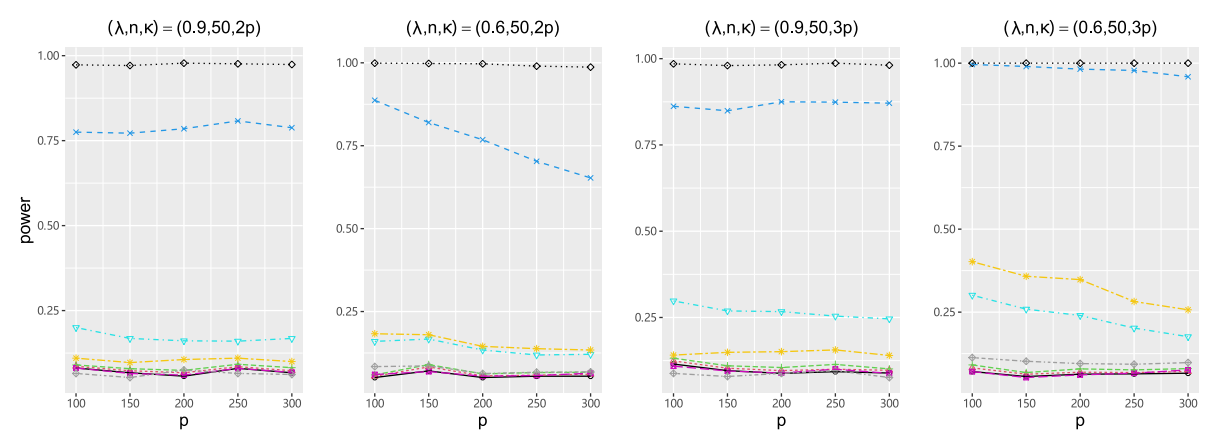

$\rightarrow W_{n}-T_{N} \cdots T_{I N} \rightarrow T_{S} \rightarrow R_{s}$

$\cdots \widetilde{W}_{n-*}-T_{C} \cdots-T_{R} \rightarrow-R_{W}$

FIG 6. The empirical power results of the nine tests under the mixed rotationally symmetric distributions at 5\% level with angular function (F3) under settings I and II.

\section{Discussion}

We have studied the general framework of directional weighted spatial sign tests for testing location of directional data under the commonly used rotationally symmetric distributions, which includes many members closely related to the existing tests. The asymptotic properties of the family of tests have been established under the rotationally symmetric distributions. Within this framework of asymptotic properties, we find that the $T_{n}\left(\omega_{\mathrm{IN}}\right)$-based test has the maximum asymptotic power in this family. Then, the power advantages of the $T_{n}\left(\omega_{\mathrm{IN}}\right)$ based test have been fully demonstrated by the theoretical and numerical results. 


\section{Appendix}

\section{Some additional simulation results}

In this subsection, we present some additional simulation results of the nine tests, as $X_{i}$ follows the non-mixed rotationally symmetric distributions.

For angular function (F1), we perform simulations under the following three settings:

(a) $\kappa=p^{2}, \delta=p^{1 / 4} / \sqrt{n \kappa}$,

(b) $\kappa=p, \delta=\sqrt{0.5+\sqrt{1+0.25}} p^{3 / 4} /(\sqrt{n} \kappa)$,

(c) $\kappa=p / n^{1 / 4}, \delta=p^{3 / 4} /(\sqrt{n} \kappa)$,

which are the same as settings (i)-(iii) used in [20], with concentration $\kappa$ and location

$$
\boldsymbol{\theta}=(1,0, \cdots, 0)^{\mathrm{T}}+\delta\left(-2 \delta, 2 \sqrt{1-\delta^{2}}, 0, \cdots, 0\right)^{\mathrm{T}} \in \mathcal{S}^{p-1} .
$$

Let $(n, p) \in\{(100,400),(200,800)\}$.

Table 5 summarizes the empirical size and power results of the nine tests by using the angular function (F1). The size results in Table 5 suggest that all these nine tests can properly control the empirical size. For power performance, the power results in Table 5 suggest that the power of the $T_{n}(\omega)$-based weighted spatial sign tests are quite similar to that of the $W_{n}$-based and $\widetilde{W}_{n}$-based tests. The power of the $R_{W}$-based and $R_{S}$-based tests are significantly smaller than those of the remaining tests.

Next, for angular functions (F2) and (F3), we let $p \in\{100,150,200,250,300\}$, $n=40$ and $\kappa \in\{2 p, 3 p\}$, respectively. The setting of $\boldsymbol{\theta}$ is the same as (A.1) for angular function (F1). We fix $\delta=\sqrt{0.65} /\left[n^{1 / 2} p^{1 / 4}\left\{\mathbb{E}\left(v_{i}^{-4}\right)\right\}^{1 / 4}\right]$ for angular function (F2), and fix $\delta=\sqrt{0.70} /\left[n^{1 / 2} p^{1 / 4}\left\{\mathbb{E}\left(v_{i}^{-4}\right)\right\}^{1 / 4}\right]$ for angular function (F3).

Table 6 summarizes the empirical size of the nine tests for angular functions (F2) and (F3), which suggests that all these tests generally control the size. In particular, we find that the empirical size of the $T_{n}(\omega)$-based weighted spatial sign tests are slightly larger than that of the $W_{n}$-based and $\widetilde{W}_{n}$-based tests. These may indicate a slight anti-conservatism of the proposed tests.

For power comparison, Figure 7 shows the power curves of $\kappa=2 p$ and $3 p$ respectively for angular function (F2), while Figure 8 shows the power curves of $\kappa=2 p$ and $3 p$ respectively for angular function (F3). From Figures 7 and 8, it can be seen that some of the proposed directional weighted spatial sign tests are more powerful, but not significantly more powerful, than the $W_{n}$-based and $\widetilde{W}_{n}$-based tests, considering the slight anti-conservatism of the proposed tests. In addition, the empirical power of the $R_{S}$-based and $R_{W}$-based tests are much lower than that of the remaining tests. In short, in the case of non-mixed rotationally symmetric distributions, the power of all tests except the $R_{S}$-based and $R_{W}$-based tests are similar. 
TABLE 5

The empirical size and power results of the nine tests under the non-mixed rotationally symmetric distributions at $5 \%$ level with angular function (F1).

\begin{tabular}{|c|c|c|c|c|c|c|c|c|c|c|c|}
\hline$n$ & $p$ & $\kappa$ & $W_{n}$ & $W_{n}$ & $T_{\mathrm{N}}$ & $T_{\mathrm{C}}$ & $T_{\mathrm{IN}}$ & $T_{\mathrm{S}}$ & $T_{\mathrm{R}}$ & $R_{W}$ & $R_{S}$ \\
\hline & \multicolumn{11}{|c|}{ size } \\
\hline 100 & 400 & $p^{2}$ & 0.054 & 0.058 & 0.062 & 0.059 & 0.060 & 0.061 & 0.059 & 0.082 & 0.076 \\
\hline 100 & 400 & $p$ & 0.051 & 0.054 & 0.056 & 0.059 & 0.061 & 0.052 & 0.057 & 0.055 & 0.057 \\
\hline 100 & 400 & $p / n^{1 / 4}$ & 0.054 & 0.057 & 0.062 & 0.062 & 0.060 & 0.064 & 0.062 & 0.066 & 0.058 \\
\hline 200 & 800 & $p^{2}$ & 0.048 & 0.050 & 0.050 & 0.051 & 0.052 & 0.047 & 0.051 & 0.050 & 0.051 \\
\hline 200 & 800 & $p$ & 0.050 & 0.051 & 0.053 & 0.052 & 0.052 & 0.054 & 0.053 & 0.052 & 0.047 \\
\hline 200 & 800 & $p / n^{1 / 4}$ & 0.060 & 0.062 & 0.064 & 0.064 & 0.063 & 0.064 & 0.064 & 0.047 & 0.050 \\
\hline \multicolumn{12}{|c|}{ power } \\
\hline 100 & 400 & $p^{2}$ & 0.814 & 0.822 & 0.831 & 0.830 & 0.824 & 0.827 & 0.832 & 0.693 & 0.514 \\
\hline 100 & 400 & $p$ & 0.826 & 0.834 & 0.840 & 0.840 & 0.845 & 0.837 & 0.841 & 0.677 & 0.495 \\
\hline 100 & 400 & $p / n^{1 / 4}$ & 0.777 & 0.785 & 0.793 & 0.791 & 0.791 & 0.793 & 0.793 & 0.631 & 0.451 \\
\hline 200 & 800 & $p^{2}$ & 0.837 & 0.841 & 0.845 & 0.845 & 0.846 & 0.844 & 0.846 & 0.654 & 0.463 \\
\hline 200 & 800 & $p$ & 0.838 & 0.842 & 0.854 & 0.847 & 0.845 & 0.852 & 0.851 & 0.690 & 0.490 \\
\hline 200 & 800 & $p / n^{1 / 4}$ & 0.794 & 0.805 & 0.810 & 0.808 & 0.807 & 0.809 & 0.808 & 0.601 & 0.411 \\
\hline
\end{tabular}

TABLE 6

The empirical size results of the nine tests under the non-mixed rotationally symmetric distributions at 5\% level with angular functions (F2) and (F3).

\begin{tabular}{rrrrrrrrrrrrr}
\hline \hline$n$ & $p$ & $\kappa$ & $W_{n}$ & $\widetilde{W}_{n}$ & $T_{\mathrm{N}}$ & $T_{\mathrm{C}}$ & $T_{\mathrm{IN}}$ & $T_{\mathrm{S}}$ & $T_{\mathrm{R}}$ & $R_{W}$ & $R_{S}$ \\
\hline 40 & 100 & 200 & 0.049 & 0.053 & 0.060 & 0.062 & 0.062 & 0.061 & 0.059 & 0.068 & 0.069 \\
40 & 100 & 300 & 0.043 & 0.046 & 0.052 & 0.056 & 0.054 & 0.056 & 0.056 & 0.056 & 0.055 \\
40 & 150 & 300 & 0.050 & 0.057 & 0.068 & 0.061 & 0.065 & 0.070 & 0.064 & 0.071 & 0.072 \\
40 & 150 & 450 & 0.044 & 0.052 & 0.060 & 0.059 & 0.061 & 0.056 & 0.058 & 0.060 & 0.060 \\
40 & 200 & 400 & 0.045 & 0.050 & 0.059 & 0.055 & 0.055 & 0.058 & 0.056 & 0.069 & 0.071 \\
40 & 200 & 600 & 0.044 & 0.051 & 0.058 & 0.056 & 0.051 & 0.058 & 0.055 & 0.060 & 0.060 \\
40 & 250 & 500 & 0.046 & 0.052 & 0.057 & 0.063 & 0.065 & 0.053 & 0.062 & 0.057 & 0.052 \\
40 & 250 & 750 & 0.041 & 0.047 & 0.057 & 0.058 & 0.057 & 0.056 & $0.058 \mathrm{~s}$ & 0.057 & 0.057 \\
40 & 300 & 600 & 0.055 & 0.058 & 0.062 & 0.061 & 0.064 & 0.063 & 0.060 & 0.057 & 0.053 \\
40 & 300 & 900 & 0.041 & 0.045 & 0.052 & 0.055 & 0.053 & 0.050 & 0.053 & 0.052 & 0.063 \\
\hline & & & & & & & $(\mathrm{F} 3)$ & & & & \\
40 & 100 & 200 & 0.042 & 0.046 & 0.058 & 0.058 & 0.057 & 0.052 & 0.058 & 0.058 & 0.057 \\
40 & 100 & 300 & 0.044 & 0.053 & 0.062 & 0.060 & 0.063 & 0.061 & 0.058 & 0.059 & 0.064 \\
40 & 150 & 300 & 0.040 & 0.048 & 0.052 & 0.055 & 0.057 & 0.062 & 0.053 & 0.067 & 0.059 \\
40 & 150 & 450 & 0.046 & 0.052 & 0.057 & 0.057 & 0.056 & 0.062 & 0.056 & 0.060 & 0.061 \\
40 & 200 & 400 & 0.044 & 0.046 & 0.052 & 0.057 & 0.056 & 0.052 & 0.057 & 0.062 & 0.068 \\
40 & 200 & 600 & 0.045 & 0.050 & 0.053 & 0.051 & 0.050 & 0.053 & 0.054 & 0.058 & 0.058 \\
40 & 250 & 500 & 0.048 & 0.054 & 0.055 & 0.056 & 0.054 & 0.054 & 0.055 & 0.056 & 0.068 \\
40 & 250 & 750 & 0.045 & 0.050 & 0.057 & 0.062 & 0.061 & 0.052 & 0.060 & 0.048 & 0.046 \\
40 & 300 & 600 & 0.056 & 0.062 & 0.071 & 0.072 & 0.073 & 0.072 & 0.074 & 0.056 & 0.050 \\
40 & 300 & 900 & 0.052 & 0.058 & 0.071 & 0.066 & 0.067 & 0.067 & 0.066 & 0.047 & 0.040 \\
\hline \hline
\end{tabular}

\section{Technical details}

If $\boldsymbol{X}_{i}$ are rotationally symmetric on $\mathcal{S}^{p-1}$ about $\boldsymbol{\theta}$, then we have the tangentnormal decomposition of $\boldsymbol{X}_{i}, \boldsymbol{X}_{i}=u_{i} \boldsymbol{\theta}+v_{i} \boldsymbol{S}_{i}$, where $u_{i}=\boldsymbol{X}_{i}^{\mathrm{T}} \boldsymbol{\theta}, v_{i}^{2}=1-u_{i}^{2}=$ $\left\|\left(\mathbf{I}_{p}-\boldsymbol{\theta} \boldsymbol{\theta}^{\mathrm{T}}\right) \boldsymbol{X}_{i}\right\|^{2}, \boldsymbol{S}_{i}=\mathbf{U}\left\{\left(\mathbf{I}_{p}-\boldsymbol{\theta} \boldsymbol{\theta}^{\mathrm{T}}\right) \boldsymbol{X}_{i}\right\}$. In addition, $\boldsymbol{S}_{i}$ are independent with $u_{i}$, and $\boldsymbol{S}_{i}$ is uniformly distributed on $\mathcal{S}^{p-1}\left(\boldsymbol{\theta}^{\perp}\right):\left\{\boldsymbol{S} \in \mathbb{R}^{p}:\|\boldsymbol{S}\|=1, \boldsymbol{S}^{\mathrm{T}} \boldsymbol{\theta}=0\right\}$. Before starting to prove, we recall Lemma B.1. in [4]. 

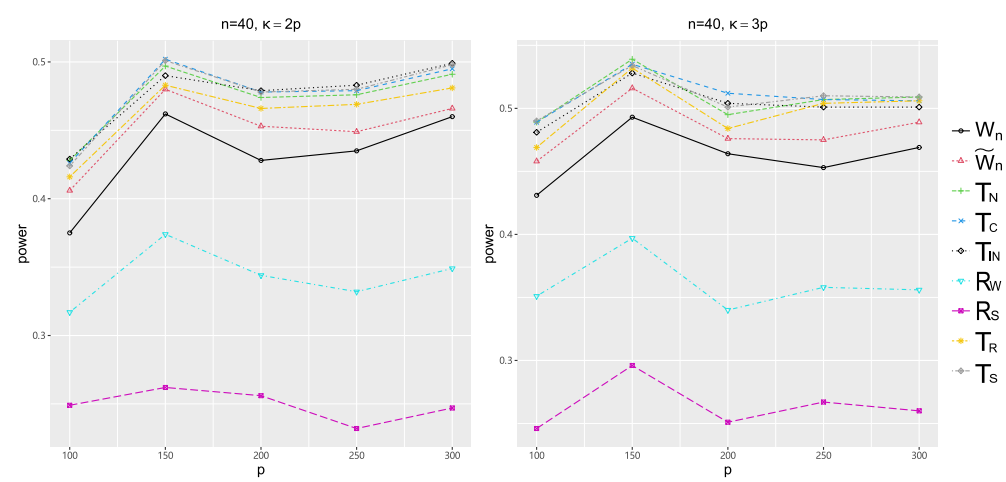

FIG 7. The power curves of nine tests under the non-mixed rotationally symmetric distributions for angular function (F2) with $\kappa=2 p$ and $3 p$.
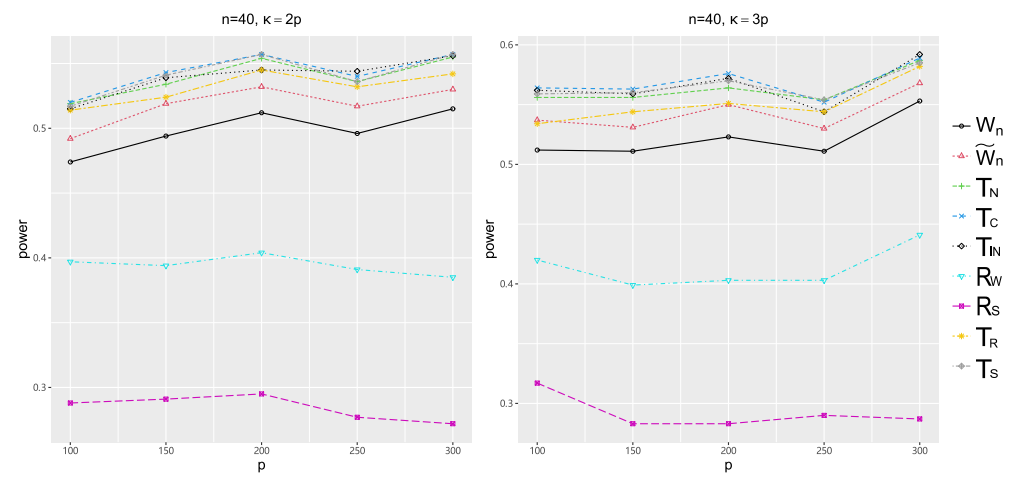

FIG 8. The power curves of nine tests under the non-mixed rotationally symmetric distributions for angular function (F3) with $\kappa=2 p$ and $3 p$.

Lemma A.1. Let $\boldsymbol{X}_{1}, \cdots, \boldsymbol{X}_{n}$ be a sequence of independent and identically distributed observations with a rotationally symmetric distribution, due to $\boldsymbol{X}_{i}=$ $u_{i} \boldsymbol{\theta}+v_{i} \boldsymbol{S}_{i}$, we have

(i) $\mathbb{E}\left(\boldsymbol{S}_{i} \boldsymbol{S}_{i}^{\mathrm{T}}\right)=\frac{1}{p-1}\left(\mathbf{I}_{p}-\boldsymbol{\theta} \boldsymbol{\theta}^{\mathrm{T}}\right)$ for any $i$
(ii) $\mathbb{E}\left\{\left(\boldsymbol{S}_{i}^{\mathrm{T}} \boldsymbol{S}_{j}\right)^{2}\right\}=\frac{1}{p-1}$ for any $i \neq j$
(iii) $\mathbb{E}\left\{\left(\boldsymbol{S}_{i}^{\mathrm{T}} \boldsymbol{S}_{j}\right)^{4}\right\}=\frac{3}{p^{2}-1}$ for any $i \neq j$

\section{Proof of Theorem 3.1}

First, it is noteworthy that under $H_{0}, \boldsymbol{X}_{i}$ are rotationally symmetric with $\boldsymbol{\theta}_{0}$, hence $\boldsymbol{S}_{i}$ and $u_{i}$ are independent. We have $\mathbb{E}\left\{T_{n}(\omega)\right\}=0$ under $H_{0}$ due to 
$\mathbb{E}\left(\boldsymbol{S}_{i}\right)=\mathbf{0}$. Then, the variance of $T_{n}(\omega)$ is

$$
\begin{aligned}
\operatorname{var}\left\{T_{n}(\omega)\right\} & =\frac{2}{n(n-1)} \mathbb{E}\left\{\omega^{2}\left(v_{i}\right) \omega^{2}\left(v_{j}\right)\left(\boldsymbol{S}_{i}^{\mathrm{T}} \boldsymbol{S}_{j}\right)^{2}\right\} \\
& =\frac{2}{n(n-1)}\left[\mathbb{E}\left\{\omega^{2}\left(v_{i}\right)\right\}\right]^{2} \mathbb{E}\left(\boldsymbol{S}_{i}^{\mathrm{T}} \boldsymbol{S}_{j}\right)^{2} \\
& =\frac{2}{n(n-1)(p-1)} b_{2}^{2}(\omega) \\
& =\sigma_{n}^{2}(\omega)\{1+o(1)\} .
\end{aligned}
$$

The normality of $T_{n}(\omega)$ has yet to be proven. Define $W_{n k}=\sum_{i=2}^{k} Z_{n i}$ where $Z_{n i}=2 /\{n(n-1)\} \sum_{j=1}^{i-1} \boldsymbol{V}_{i}^{\mathrm{T}} \boldsymbol{V}_{j}, \boldsymbol{V}_{i}=\omega\left(v_{i}\right) \boldsymbol{S}_{i}$. Let $\mathbf{A}=\mathbb{E}\left(\boldsymbol{V}_{i} \boldsymbol{V}_{i}^{\mathrm{T}}\right)$. Note that $\mathbf{A}=b_{2}(\omega)\left(\mathbf{I}_{p}-\boldsymbol{\theta}_{0} \boldsymbol{\theta}_{0}^{\mathrm{T}}\right) /(p-1)$ due to Lemma A.1. Let $\mathcal{F}_{n, i} \doteq \sigma\left\{\boldsymbol{V}_{1}, \cdots, \boldsymbol{V}_{i}\right\}$ be the $\sigma$-field generated by $\left\{\boldsymbol{V}_{j}, j \leq i\right\}$. Obviously, $\mathbb{E}\left(Z_{n i} \mid \mathcal{F}_{n, i-1}\right)=0$ and it follows that $\left\{W_{n k}, \mathcal{F}_{n, k} ; 2 \leq k \leq n\right\}$ is a zero mean martingale. According to the Martingale central limit theorem in [9], we only need to show

$$
\frac{\sum_{j=2}^{n} \mathbb{E}\left(Z_{n j}^{2} \mid \mathcal{F}_{n, j-1}\right)}{\sigma_{n}^{2}(\omega)} \stackrel{p}{\rightarrow} 1
$$

and

$$
\mathbb{E}\left\{\sum_{j=2}^{n} \mathbb{E}\left(Z_{n j}^{4} \mid \mathcal{F}_{n, j-1}\right)\right\}=o\left\{\sigma_{n}^{4}(\omega)\right\} .
$$

It can be shown that

$$
\begin{aligned}
\sum_{j=2}^{n} \mathbb{E}\left(Z_{n j}^{2} \mid \mathcal{F}_{n, j-1}\right) & =\sum_{j=2}^{n} \mathbb{E}\left[\left\{\frac{2}{n(n-1)} \sum_{i=1}^{j-1} \boldsymbol{V}_{i}^{\mathrm{T}} \boldsymbol{V}_{j}\right\}^{2} \mid \mathcal{F}_{n, j-1}\right] \\
& =\sum_{j=2}^{n} \frac{4}{n^{2}(n-1)^{2}} \mathbb{E}\left\{\left(\sum_{i_{1}=1}^{j-1} \sum_{i_{2}=1}^{j-1} \boldsymbol{V}_{i_{1}}^{\mathrm{T}} \boldsymbol{V}_{j} \boldsymbol{V}_{i_{2}}^{\mathrm{T}} \boldsymbol{V}_{j}\right) \mid \mathcal{F}_{n, j-1}\right\} \\
& =\sum_{j=2}^{n} \frac{4}{n^{2}(n-1)^{2}} \sum_{i_{1}=1}^{j-1} \sum_{i_{2}=1}^{j-1} \boldsymbol{V}_{i_{1}}^{\mathrm{T}} \mathbb{E}\left(\boldsymbol{V}_{j} \boldsymbol{V}_{j}^{\mathrm{T}}\right) \boldsymbol{V}_{i_{2}} \\
& =C_{n 1}+C_{n 2},
\end{aligned}
$$

where

$$
C_{n 1}=\frac{4}{n^{2}(n-1)^{2}} \sum_{j=2}^{n} \sum_{i=1}^{j-1} \boldsymbol{V}_{i}^{\mathrm{T}} \mathbf{A} \boldsymbol{V}_{i} \text { and } C_{n 2}=\frac{8}{n^{2}(n-1)^{2}} \sum_{j=2}^{n} \sum_{i_{1}<i_{2}}^{j-1} \sum_{i_{1}}^{j-1} \boldsymbol{V}^{\mathrm{T}} \mathbf{\boldsymbol { V } _ { i _ { 2 } }} .
$$

We first consider $C_{n 1}$. Due to $\mathbf{A}=\mathbb{E}\left(\boldsymbol{V}_{i} \boldsymbol{V}_{i}^{\mathrm{T}}\right)$, we have

$$
\mathbb{E}\left(C_{n 1}\right)=\frac{2}{n(n-1)} \mathbb{E}\left(\boldsymbol{V}_{i}^{\mathrm{T}} \mathbf{A} \boldsymbol{V}_{i}\right)
$$




$$
\begin{aligned}
& =\frac{2}{n(n-1)} \operatorname{tr}\left\{\mathbb{E}\left(\mathbf{A} \boldsymbol{V}_{i} \boldsymbol{V}_{i}^{\mathrm{T}}\right)\right\} \\
& =\frac{2}{n(n-1)} \operatorname{tr}\left(\mathbf{A}^{2}\right) \\
& =\sigma_{n}^{2}(\omega)\{1+o(1)\},
\end{aligned}
$$

where $\mathbf{A}=b_{2}(\omega)\left(\mathbf{I}_{p}-\boldsymbol{\theta}_{0} \boldsymbol{\theta}_{0}^{\mathrm{T}}\right) /(p-1)$ and $\boldsymbol{S}_{i}=\left(\mathbf{I}_{p}-\boldsymbol{\theta}_{0} \boldsymbol{\theta}_{0}^{\mathrm{T}}\right) \boldsymbol{X}_{i} / v_{i}$. We also obtain

$$
\begin{aligned}
\boldsymbol{S}_{i}^{\mathrm{T}} \mathbf{A} \boldsymbol{S}_{i} & \left.\left.=\frac{1}{v_{i}^{2}(p-1)} b_{2}(\omega) \boldsymbol{X}_{i}^{\mathrm{T}}\left(\mathbf{I}_{p}-\boldsymbol{\theta}_{0} \boldsymbol{\theta}_{0}^{\mathrm{T}}\right)\left(\mathbf{I}_{p}-\boldsymbol{\theta}_{0} \boldsymbol{\theta}_{0}^{\mathrm{T}}\right)\right)\left(\mathbf{I}_{p}-\boldsymbol{\theta}_{0} \boldsymbol{\theta}_{0}^{\mathrm{T}}\right)\right) \boldsymbol{X}_{i} \\
& =\frac{1}{p-1} b_{2}(\omega) .
\end{aligned}
$$

Thus,

$$
\left(\boldsymbol{S}_{i}^{\mathrm{T}} \mathbf{A} \boldsymbol{S}_{i}\right)^{2}=\frac{b_{2}^{2}(\omega)}{(p-1)^{2}}
$$

In addition,

$$
\begin{aligned}
\operatorname{var}\left(C_{n 1}\right) & =\operatorname{var}\left(\frac{4}{n^{2}(n-1)^{2}} \sum_{j=2}^{n} \sum_{i=1}^{j-1} \boldsymbol{V}_{i}^{\mathrm{T}} \mathbf{A} \boldsymbol{V}_{i}\right) \\
& =\operatorname{var}\left\{\frac{4}{n^{2}(n-1)^{2}} \sum_{j=2}^{n} \sum_{i=1}^{j-1} \frac{1}{p-1} b_{2}(\omega) \omega^{2}\left(v_{i}\right)\right\} \\
& =\operatorname{var}\left\{\frac{4}{n^{2}(n-1)^{2}} \sum_{i=1}^{n} \sum_{j=i+1}^{n} \frac{1}{p-1} b_{2}(\omega) \omega^{2}\left(v_{i}\right)\right\} \\
& =\operatorname{var}\left\{\frac{4}{n^{2}(n-1)^{2}} \sum_{i=1}^{n} \frac{n-i}{p-1} b_{2}(\omega) \omega^{2}\left(v_{i}\right)\right\} \\
& \leq O\left\{n^{-5}(p-1)^{-2}\right\} b_{4}(\omega) b_{2}^{2}(\omega) \\
& =o\left\{\sigma_{n}^{4}(\omega)\right\},
\end{aligned}
$$

where we used Condition (C1) in the last equality. Then, we have

$$
C_{n 1} / \sigma_{n}^{2}(\omega) \stackrel{p}{\rightarrow} 1 .
$$

Similarly,

$$
\begin{aligned}
\mathbb{E}\left(C_{n 2}^{2}\right) & =\mathbb{E}\left\{\frac{8}{n^{2}(n-1)^{2}} \sum_{j=2}^{n} \sum_{i_{1}<i_{2}}^{j-1} \sum_{i_{1}}^{j-1} \mathbf{A} \boldsymbol{V}_{i_{2}}\right\}^{2} \\
& =O\left(n^{-8}\right) \mathbb{E}\left(\sum_{j_{1}=2}^{n} \sum_{j_{2}=2}^{n} \sum_{i_{1}<i_{2}}^{j_{1}-1} \sum_{i_{1}-1}^{j_{2}-1} \sum_{i_{3}<i_{4}}^{j_{2}-1} \boldsymbol{V}_{i_{1}}^{\mathrm{T}} \mathbf{A} \boldsymbol{V}_{i_{2}} \boldsymbol{V}_{i_{3}}^{\mathrm{T}} \mathbf{A} \boldsymbol{V}_{i_{4}}\right)
\end{aligned}
$$




$$
\begin{aligned}
& =O\left(n^{-8}\right) \mathbb{E}\left\{\sum_{j_{1}<j_{2}}^{n} \sum_{i_{1}<i_{2}}^{n} \sum_{j_{1}-1}^{j_{1}-1}\left(\boldsymbol{V}_{i_{1}}^{\mathrm{T}} \mathbf{A} \boldsymbol{V}_{i_{2}}\right)^{2}\right\} \\
& =O\left(n^{-8}\right) \mathbb{E}\left\{\sum_{j_{2}=2 j_{1}=1}^{n} \sum_{j_{1}<i_{1}}^{j_{2}-1 j_{1}-1} \sum_{j_{1}-1}\left(\boldsymbol{V}_{i_{1}}^{\mathrm{T}} \mathbf{A} \boldsymbol{V}_{i_{2}}\right)^{2}\right\} \\
& =O\left(n^{-4}\right) \mathbb{E}\left\{\left(\boldsymbol{V}_{i}^{\mathrm{T}} \mathbf{A} \boldsymbol{V}_{j}\right)^{2}\right\} \\
& =O\left(n^{-4}\right) \mathbb{E}\left\{\omega^{2}\left(v_{i}\right) \omega^{2}\left(v_{j}\right)\left(\boldsymbol{S}_{i}^{\mathrm{T}} \mathbf{A} \boldsymbol{S}_{j}\right)^{2}\right\} \\
& =O\left(n^{-4}\right) b_{2}^{2}(\omega) \operatorname{tr}\left\{\mathbb{E}\left(\boldsymbol{S}_{i}^{\mathrm{T}} \mathbf{A} \boldsymbol{S}_{j} \boldsymbol{S}_{j}^{\mathrm{T}} \mathbf{A} \boldsymbol{S}_{i}\right)\right\} \\
& =O\left(n^{-4} p^{-3}\right) b_{2}^{4}(\omega)=o\left\{\sigma_{n}^{4}(\omega)\right\},
\end{aligned}
$$

where $i \neq j$ in the fourth equality and we used $\mathbf{A}=(p-1)^{-1} b_{2}(\omega)\left(\mathbf{I}_{p}-\boldsymbol{\theta}_{0} \boldsymbol{\theta}_{0}^{\mathrm{T}}\right)$ and Lemma A.1 (ii) in the seventh equality. Then (A.2) holds.

Next, we only need to show

$$
\mathbb{E}\left\{\sum_{j=2}^{n} \mathbb{E}\left(Z_{n j}^{4} \mid \mathcal{F}_{n, j-1}\right)\right\}=o\left\{\sigma_{n}^{4}(\omega)\right\}
$$

Note that

$$
\mathbb{E}\left\{\sum_{j=2}^{n} \mathbb{E}\left(Z_{n j}^{4} \mid \mathcal{F}_{n, j-1}\right)\right\}=\sum_{j=2}^{n} \mathbb{E}\left(Z_{n j}^{4}\right)=O\left(n^{-8}\right) \sum_{j=2}^{n} \mathbb{E}\left(\sum_{i=1}^{j-1} \boldsymbol{V}_{j}^{\mathrm{T}} \boldsymbol{V}_{i}\right)^{4}
$$

can be decomposed as $Q+P$, where

$$
\begin{aligned}
& Q=O\left(n^{-8}\right) \sum_{j=2}^{n} \sum_{i_{1}<i_{2}}^{j-1} \sum_{j}^{j-1} \mathbb{E}\left(\boldsymbol{V}_{j}^{\mathrm{T}} \boldsymbol{V}_{i_{1}} \boldsymbol{V}_{i_{1}}^{\mathrm{T}} \boldsymbol{V}_{j} \boldsymbol{V}_{j}^{\mathrm{T}} \boldsymbol{V}_{i_{2}} \boldsymbol{V}_{i_{2}}^{\mathrm{T}} \boldsymbol{V}_{j}\right), \\
& P=O\left(n^{-8}\right) \sum_{j=2}^{n} \sum_{i=1}^{j-1} \mathbb{E}\left\{\left(\boldsymbol{V}_{j}^{\mathrm{T}} \boldsymbol{V}_{i}\right)^{4}\right\} .
\end{aligned}
$$

Because

$$
\begin{aligned}
Q & =O\left(n^{-5}\right) \mathbb{E}\left(\boldsymbol{V}_{j}^{\mathrm{T}} \boldsymbol{V}_{i_{1}} \boldsymbol{V}_{i_{1}}^{\mathrm{T}} \boldsymbol{V}_{j} \boldsymbol{V}_{j}^{\mathrm{T}} \boldsymbol{V}_{i_{2}} \boldsymbol{V}_{i_{2}}^{\mathrm{T}} \boldsymbol{V}_{j}\right) \\
& =O\left(n^{-5}\right) \operatorname{tr}\left\{\mathbb{E}\left(\boldsymbol{V}_{i_{1}} \boldsymbol{V}_{i_{1}}^{\mathrm{T}} \boldsymbol{V}_{j} \boldsymbol{V}_{j}^{\mathrm{T}} \boldsymbol{V}_{i_{2}} \boldsymbol{V}_{i_{2}}^{\mathrm{T}} \boldsymbol{V}_{j} \boldsymbol{V}_{j}^{\mathrm{T}}\right)\right\} \\
& =O\left(n^{-5}\right) \operatorname{tr}\left[\mathbb{E}\left\{\mathbb{E}\left(\boldsymbol{V}_{i_{1}} \boldsymbol{V}_{i_{1}}^{\mathrm{T}} \boldsymbol{V}_{j} \boldsymbol{V}_{j}^{\mathrm{T}} \boldsymbol{V}_{i_{2}} \boldsymbol{V}_{i_{2}}^{\mathrm{T}} \boldsymbol{V}_{j} \boldsymbol{V}_{j}^{\mathrm{T}} \mid \boldsymbol{V}_{j}\right)\right\}\right] \\
& =O\left(n^{-5}\right) \operatorname{tr}\left\{\mathbb{E}\left(\mathbf{A} \boldsymbol{V}_{j} \boldsymbol{V}_{j}^{\mathrm{T}} \mathbf{A} \boldsymbol{V}_{j} \boldsymbol{V}_{j}^{\mathrm{T}}\right)\right\} \\
& =O\left(n^{-5}\right) \mathbb{E}\left\{\left(\boldsymbol{V}_{j}^{\mathrm{T}} \mathbf{A} \boldsymbol{V}_{j}\right)^{2}\right\},
\end{aligned}
$$

hence, $Q=O\left\{n^{-5}(p-1)^{-2}\right\} b_{4}(\omega) b_{2}^{2}(\omega)=o\left\{\sigma_{n}^{4}(\omega)\right\}$ due to (A.4) and Condition (C1). Similarly, due to Lemma A.1 (iii), we can show that

$$
P=O\left(n^{-6}\right) \mathbb{E}\left\{\left(\boldsymbol{V}_{j}^{\mathrm{T}} \boldsymbol{V}_{i}\right)^{4}\right\}
$$




$$
\begin{aligned}
& =O\left(n^{-6}\right) \mathbb{E}\left\{\omega^{4}\left(v_{i}\right) \omega^{4}\left(v_{j}\right)\left(\boldsymbol{S}_{j}^{\mathrm{T}} \boldsymbol{S}_{i}\right)^{4}\right\} \\
& =O\left(n^{-6}\right) b_{4}^{2}(\omega) \mathbb{E}\left\{\left(\boldsymbol{S}_{j}^{\mathrm{T}} \boldsymbol{S}_{i}\right)^{4}\right\} \\
& =O\left(n^{-6} p^{-2}\right) b_{2}^{4}(\omega) \\
& =O\left\{n^{-2} \sigma_{n}^{4}(\omega)\right\}
\end{aligned}
$$

Then, we complete the proof.

\section{Proof of Theorem 3.2}

We have

$$
\begin{aligned}
\widehat{\sigma}_{n}^{2}(\omega) & =2 n^{-4} p^{-1} \sum_{i \neq j} \sum_{j} \omega\left(\left\|\left(\mathbf{I}_{p}-\boldsymbol{\theta}_{0} \boldsymbol{\theta}_{0}^{\mathrm{T}}\right) \boldsymbol{X}_{i}\right\|\right)^{2} \omega\left(\left\|\left(\mathbf{I}_{p}-\boldsymbol{\theta}_{0} \boldsymbol{\theta}_{0}^{\mathrm{T}}\right) \boldsymbol{X}_{j}\right\|\right)^{2} \\
& =2 n^{-4} p^{-1} \sum_{i \neq j} \omega\left(v_{i}\right)^{2} \omega\left(v_{j}\right)^{2} .
\end{aligned}
$$

First of all,

$$
\mathbb{E}\left(\widehat{\sigma}_{n}^{2}(\omega)\right)=2 n^{-2} p^{-1} b_{2}^{2}(\omega)=\sigma_{n}^{2}(\omega)\{1+o(1)\} .
$$

Next, we have

$$
\begin{aligned}
\operatorname{var}\left\{\widehat{\sigma}_{n}^{2}(\omega)\right\}= & \mathbb{E}\left[\left\{2 n^{-4} p^{-1} \sum_{i \neq j} \sum_{j} \omega\left(v_{i}\right)^{2} \omega\left(v_{j}\right)^{2}\right\}^{2}\right] \\
& -\left[\mathbb{E}\left\{2 n^{-4} p^{-1} \sum_{i \neq j} \omega\left(v_{i}\right)^{2} \omega\left(v_{j}\right)^{2}\right\}\right]^{2} \\
= & O\left(n^{-6} p^{-2}\right) b_{4}^{2}(\omega)+O\left(n^{-5} p^{-2}\right) b_{2}^{2}(\omega) b_{4}(\omega) \\
= & o\left\{\sigma_{n}^{4}(\omega)\right\},
\end{aligned}
$$

where the last equality is due to Condition (C1), which leads to

$$
\widehat{\sigma}_{n}^{2}(\omega) / \sigma_{n}^{2}(\omega) \stackrel{p}{\rightarrow} 1 .
$$

\section{Proof of Theorem 3.3}

We have

$$
\begin{aligned}
U\left\{\left(\mathbf{I}_{p}-\boldsymbol{\theta}_{0} \boldsymbol{\theta}_{0}^{\mathrm{T}}\right) \boldsymbol{X}_{i}\right\}= & U\left\{\left(\mathbf{I}_{p}-\boldsymbol{\theta} \boldsymbol{\theta}^{\mathrm{T}}\right) \boldsymbol{X}_{i}+\left(\boldsymbol{\theta} \boldsymbol{\theta}^{\mathrm{T}}-\boldsymbol{\theta}_{0} \boldsymbol{\theta}_{0}^{\mathrm{T}}\right) \boldsymbol{X}_{i}\right\} \\
= & \left\{\left(\mathbf{I}_{p}-\boldsymbol{\theta} \boldsymbol{\theta}^{\mathrm{T}}\right) \boldsymbol{X}_{i}+\left(\boldsymbol{\theta} \boldsymbol{\theta}^{\mathrm{T}}-\boldsymbol{\theta}_{0} \boldsymbol{\theta}_{0}^{\mathrm{T}}\right) \boldsymbol{X}_{i}\right\} \\
& \times\left\{v_{i}^{2}+\boldsymbol{X}_{i}^{\mathrm{T}}\left(\boldsymbol{\theta} \boldsymbol{\theta}^{\mathrm{T}}-\boldsymbol{\theta}_{0} \boldsymbol{\theta}_{0}^{\mathrm{T}}\right) \boldsymbol{X}_{i}\right\}^{-1 / 2} \\
= & \left\{\boldsymbol{S}_{i}+v_{i}^{-1}\left(\boldsymbol{\theta} \boldsymbol{\theta}^{\mathrm{T}}-\boldsymbol{\theta}_{0} \boldsymbol{\theta}_{0}^{\mathrm{T}}\right) \boldsymbol{X}_{i}\right\}
\end{aligned}
$$




$$
\begin{gathered}
\times\left\{1+v_{i}^{-2} \boldsymbol{X}_{i}^{\mathrm{T}}\left(\boldsymbol{\theta} \boldsymbol{\theta}^{\mathrm{T}}-\boldsymbol{\theta}_{0} \boldsymbol{\theta}_{0}^{\mathrm{T}}\right) \boldsymbol{X}_{i}\right\}^{-1 / 2} \\
=\left\{\boldsymbol{S}_{i}+v_{i}^{-1}\left(\boldsymbol{\theta} \boldsymbol{\theta}^{\mathrm{T}}-\boldsymbol{\theta}_{0} \boldsymbol{\theta}_{0}^{\mathrm{T}}\right) \boldsymbol{X}_{i}\right\}\left\{1+\alpha_{i}\right\}^{-1 / 2},
\end{gathered}
$$

where $\alpha_{i}=v_{i}^{-2} \boldsymbol{X}_{i}^{\mathrm{T}}\left(\boldsymbol{\theta} \boldsymbol{\theta}^{\mathrm{T}}-\boldsymbol{\theta}_{0} \boldsymbol{\theta}_{0}^{\mathrm{T}}\right) \boldsymbol{X}_{i}$. Note that

$$
\left\|\left(\mathbf{I}_{p}-\boldsymbol{\theta}_{0} \boldsymbol{\theta}_{0}^{\mathrm{T}}\right) \boldsymbol{X}_{i}\right\|=v_{i}\left(1+\alpha_{i}\right)^{1 / 2} .
$$

Thus,

$$
\begin{aligned}
& T_{n}(\omega) \\
= & \frac{2}{n(n-1)} \sum_{i<j} \sum_{j} \omega\left\{v_{i}\left(1+\alpha_{i}\right)^{1 / 2}\right\} \omega\left\{v_{j}\left(1+\alpha_{j}\right)^{1 / 2}\right\} U\left(\left(\mathbf{I}_{p}-\boldsymbol{\theta}_{0} \boldsymbol{\theta}_{0}^{\mathrm{T}}\right) \boldsymbol{X}_{i}\right)^{\mathrm{T}} \\
& \times U\left(\left(\mathbf{I}_{p}-\boldsymbol{\theta}_{0} \boldsymbol{\theta}_{0}^{\mathrm{T}}\right) \boldsymbol{X}_{j}\right) \\
= & \frac{2}{n(n-1)} \sum_{i<j} \sum_{i} \omega\left\{v_{i}\left(1+\alpha_{i}\right)^{1 / 2}\right\} \omega\left\{v_{j}\left(1+\alpha_{j}\right)^{1 / 2}\right\} \\
& \times\left\{\boldsymbol{S}_{i}+v_{i}^{-1}\left(\boldsymbol{\theta} \boldsymbol{\theta}^{\mathrm{T}}-\boldsymbol{\theta}_{0} \boldsymbol{\theta}_{0}^{\mathrm{T}}\right) \boldsymbol{X}_{i}\right\}^{\mathrm{T}}\left\{\boldsymbol{S}_{j}+v_{j}^{-1}\left(\boldsymbol{\theta}^{\mathrm{T}}-\boldsymbol{\theta}_{0} \boldsymbol{\theta}_{0}^{\mathrm{T}}\right) \boldsymbol{X}_{j}\right\} \\
& \times\left(1+\alpha_{i}\right)^{-1 / 2}\left(1+\alpha_{j}\right)^{-1 / 2} \\
= & \frac{2}{n(n-1)} \sum_{i<j} \sum_{i} \boldsymbol{V}_{i}^{\mathrm{T}} \boldsymbol{V}_{j}+A_{1}+A_{2}+A_{3}+A_{4}+A_{5}+A_{6},
\end{aligned}
$$

where

$$
\begin{aligned}
A_{1}= & \frac{2}{n(n-1)} \sum_{i<j} \sum_{i}\left[\omega\left\{v_{i}\left(1+\alpha_{i}\right)^{1 / 2}\right\} \omega\left\{v_{j}\left(1+\alpha_{j}\right)^{1 / 2}\right\}-\omega\left(v_{i}\right) \omega\left(v_{j}\right)\right] \boldsymbol{S}_{i}^{\mathrm{T}} \boldsymbol{S}_{j}, \\
A_{2}= & \left.\frac{2}{n(n-1)} \sum_{i<j} \sum_{i<} \omega v_{i}\left(1+\alpha_{i}\right)^{1 / 2}\right\} \omega\left\{v_{j}\left(1+\alpha_{j}\right)^{1 / 2}\right\} \\
& \times\left\{v_{i}^{-1} \boldsymbol{X}_{i}^{\mathrm{T}}\left(\boldsymbol{\theta} \boldsymbol{\theta}^{\mathrm{T}}-\boldsymbol{\theta}_{0} \boldsymbol{\theta}_{0}^{\mathrm{T}}\right) \boldsymbol{S}_{j}+v_{j}^{-1} \boldsymbol{S}_{i}^{\mathrm{T}}\left(\boldsymbol{\theta}^{\mathrm{T}}-\boldsymbol{\theta}_{0} \boldsymbol{\theta}_{0}^{\mathrm{T}}\right) \boldsymbol{X}_{j}\right\}, \\
A_{3}= & \frac{2}{n(n-1)} \sum_{i<j} \sum_{i} \omega\left\{v_{i}\left(1+\alpha_{i}\right)^{1 / 2}\right\} \omega\left\{v_{j}\left(1+\alpha_{j}\right)^{1 / 2}\right\} \\
& \times\left\{v_{i}^{-1} v_{j}^{-1} \boldsymbol{X}_{i}^{\mathrm{T}}\left(\boldsymbol{\theta} \boldsymbol{\theta}^{\mathrm{T}}+\boldsymbol{\theta}_{0} \boldsymbol{\theta}_{0}^{\mathrm{T}}-\boldsymbol{\theta} \boldsymbol{\theta}^{\mathrm{T}} \boldsymbol{\theta}_{0} \boldsymbol{\theta}_{0}^{\mathrm{T}}-\boldsymbol{\theta}_{0} \boldsymbol{\theta}_{0}^{\mathrm{T}} \boldsymbol{\theta} \boldsymbol{\theta}^{\mathrm{T}}\right) \boldsymbol{X}_{j}\right\}, \\
A_{4}= & \frac{2}{n(n-1)} \sum_{i<j} \sum_{i<} \omega\left\{v_{i}\left(1+\alpha_{i}\right)^{1 / 2}\right\} \omega\left\{v_{j}\left(1+\alpha_{j}\right)^{1 / 2}\right\} \boldsymbol{S}_{i}^{\mathrm{T}} \boldsymbol{S}_{j} \\
& \times\left\{\left(1+\alpha_{i}\right)^{-1 / 2}\left(1+\alpha_{j}\right)^{-1 / 2}-1\right\}, \\
A_{5}= & \frac{2}{n(n-1)} \sum_{i<j} \sum_{i} \omega\left\{v_{i}\left(1+\alpha_{i}\right)^{1 / 2}\right\} \omega\left\{v_{j}\left(1+\alpha_{j}\right)^{1 / 2}\right\} \\
& \times\left\{v_{i}^{-1} \boldsymbol{X}_{i}^{\mathrm{T}}\left(\boldsymbol{\theta} \boldsymbol{\theta}^{\mathrm{T}}-\boldsymbol{\theta}_{0} \boldsymbol{\theta}_{0}^{\mathrm{T}}\right) \boldsymbol{S}_{j}+v_{j}^{-1} \boldsymbol{S}_{i}^{\mathrm{T}}\left(\boldsymbol{\theta} \boldsymbol{\theta}^{\mathrm{T}}-\boldsymbol{\theta}_{0} \boldsymbol{\theta}_{0}^{\mathrm{T}}\right) \boldsymbol{X}_{j}\right\} \\
& \times\left\{\left(1+\alpha_{i}\right)^{-1 / 2}\left(1+\alpha_{j}\right)^{-1 / 2}-1\right\}, \\
A_{6}= & \frac{2}{n(n-1)} \sum_{i<j} \sum \omega\left\{v_{i}\left(1+\alpha_{i}\right)^{-1 / 2}\right\} \omega\left\{v_{j}\left(1+\alpha_{j}\right)^{-1 / 2}\right\}\left\{v_{i}^{-1} v_{j}^{-1} \boldsymbol{X}_{i}^{\mathrm{T}}\right.
\end{aligned}
$$




$$
\begin{aligned}
& \left.\times\left(\boldsymbol{\theta} \boldsymbol{\theta}^{\mathrm{T}}+\boldsymbol{\theta}_{0} \boldsymbol{\theta}_{0}^{\mathrm{T}}-\boldsymbol{\theta} \boldsymbol{\theta}^{\mathrm{T}} \boldsymbol{\theta}_{0} \boldsymbol{\theta}_{0}^{\mathrm{T}}-\boldsymbol{\theta}_{0} \boldsymbol{\theta}_{0}^{\mathrm{T}} \boldsymbol{\theta} \boldsymbol{\theta}^{\mathrm{T}}\right) \boldsymbol{X}_{j}\right\} \\
& \times\left\{\left(1+\alpha_{i}\right)^{-1 / 2}\left(1+\alpha_{j}\right)^{-1 / 2}-1\right\} .
\end{aligned}
$$

Because of the tangent-normal decomposition, we have

$$
\alpha_{i}=v_{i}^{-2} u_{i}^{2}-v_{i}^{-2} u_{i}^{2} \boldsymbol{\theta}^{\mathrm{T}} \boldsymbol{\theta}_{0} \boldsymbol{\theta}_{0}^{\mathrm{T}} \boldsymbol{\theta}-2 v_{i}^{-1} u_{i} \boldsymbol{S}_{i}^{\mathrm{T}} \boldsymbol{\theta}_{0} \boldsymbol{\theta}_{0}^{\mathrm{T}} \boldsymbol{\theta}-\left(\boldsymbol{S}_{i}^{\mathrm{T}} \boldsymbol{\theta}_{0}\right)^{2},
$$

then,

$$
\begin{aligned}
\mathbb{E}\left(\alpha_{i}\right) & =\mathbb{E}\left(v_{i}^{-2} u_{i}^{2}\right)\left\{\boldsymbol{\theta}^{\mathrm{T}}\left(\boldsymbol{\theta} \boldsymbol{\theta}^{\mathrm{T}}-\boldsymbol{\theta}_{0} \boldsymbol{\theta}_{0}^{\mathrm{T}}\right) \boldsymbol{\theta}\right\}-\mathbb{E}\left\{\left(\boldsymbol{S}_{i}^{\mathrm{T}} \boldsymbol{\theta}_{0}\right)^{2}\right\} \\
& =\mathbb{E}\left(v_{i}^{-2} u_{i}^{2}\right)\left\{\boldsymbol{\theta}^{\mathrm{T}}\left(\boldsymbol{\theta} \boldsymbol{\theta}^{\mathrm{T}}-\boldsymbol{\theta}_{0} \boldsymbol{\theta}_{0}^{\mathrm{T}}\right) \boldsymbol{\theta}\right\}-\mathbb{E}\left[\left\{\boldsymbol{S}_{i}^{\mathrm{T}}\left(\boldsymbol{\theta}_{0}-\boldsymbol{\theta}\right)\right\}^{2}\right] \\
& \leq \mathbb{E}\left(v_{i}^{-2} u_{i}^{2}\right)\left\{\boldsymbol{\theta}^{\mathrm{T}}\left(\boldsymbol{\theta} \boldsymbol{\theta}^{\mathrm{T}}-\boldsymbol{\theta}_{0} \boldsymbol{\theta}_{0}^{\mathrm{T}}\right) \boldsymbol{\theta}\right\}-\left\|\boldsymbol{\theta}_{0}-\boldsymbol{\theta}\right\|^{2} \\
& \leq \sqrt{\mathbb{E}\left(v_{i}^{-4} u_{i}^{4}\right)}\left\{\boldsymbol{\theta}^{\mathrm{T}}\left(\boldsymbol{\theta} \boldsymbol{\theta}^{\mathrm{T}}-\boldsymbol{\theta}_{0} \boldsymbol{\theta}_{0}^{\mathrm{T}}\right) \boldsymbol{\theta}\right\} .
\end{aligned}
$$

According to $\boldsymbol{\theta}^{\mathrm{T}}\left(\boldsymbol{\theta} \boldsymbol{\theta}^{\mathrm{T}}-\boldsymbol{\theta}_{0} \boldsymbol{\theta}_{0}^{\mathrm{T}}\right) \boldsymbol{\theta}=1-\left(\boldsymbol{\theta}^{\mathrm{T}} \boldsymbol{\theta}_{0}\right)^{2}=\left\|\boldsymbol{\theta}-\boldsymbol{\theta}_{0}\right\|^{2}-\left\|\boldsymbol{\theta}-\boldsymbol{\theta}_{0}\right\|^{4} / 4$ and Condition (C2), we have $\mathbb{E}\left(\alpha_{i}\right)=o(1)$, then we concentrate on the variance of $\alpha_{i}$, where

$$
\begin{aligned}
\operatorname{var}\left(\alpha_{i}\right) \leq & \mathbb{E}\left(\alpha_{i}^{2}\right) \\
\leq & 4 \mathbb{E}\left(v_{i}^{-4} u_{i}^{4}\right)\left\{\boldsymbol{\theta}^{\mathrm{T}}\left(\boldsymbol{\theta} \boldsymbol{\theta}^{\mathrm{T}}-\boldsymbol{\theta}_{0} \boldsymbol{\theta}_{0}^{\mathrm{T}}\right) \boldsymbol{\theta}\right\}^{2}+4 \mathbb{E}\left(\boldsymbol{S}_{i}^{\mathrm{T}} \boldsymbol{\theta}_{0}\right)^{4} \\
& +8 \mathbb{E}\left(u_{i} v_{i}^{-1} \boldsymbol{S}_{i}^{\mathrm{T}} \boldsymbol{\theta}_{0} \boldsymbol{\theta}_{0}^{\mathrm{T}} \boldsymbol{\theta}\right)^{2} \\
= & 4 \mathbb{E}\left(v_{i}^{-4} u_{i}^{4}\right)\left\{\boldsymbol{\theta}^{\mathrm{T}}\left(\boldsymbol{\theta} \boldsymbol{\theta}^{\mathrm{T}}-\boldsymbol{\theta}_{0} \boldsymbol{\theta}_{0}^{\mathrm{T}}\right) \boldsymbol{\theta}\right\}^{2}+4 \mathbb{E}\left(\boldsymbol{S}_{i}^{\mathrm{T}} \boldsymbol{\theta}_{0}-\boldsymbol{S}_{i}^{\mathrm{T}} \boldsymbol{\theta}\right)^{4} \\
& +8 \mathbb{E}\left(u_{i}^{2} v_{i}^{-2}\right) \operatorname{tr}\left\{\boldsymbol{\theta}_{0} \boldsymbol{\theta}_{0}^{\mathrm{T}} \boldsymbol{\theta} \boldsymbol{\theta}^{\mathrm{T}} \boldsymbol{\theta}_{0} \boldsymbol{\theta}_{0}^{\mathrm{T}} \mathbb{E}\left(\boldsymbol{S}_{i} \boldsymbol{S}_{i}^{\mathrm{T}}\right)\right\} \\
= & o(1)+4 \mathbb{E}\left\{\boldsymbol{S}_{i}^{\mathrm{T}}\left(\boldsymbol{\theta}_{0}-\boldsymbol{\theta}\right)\right\}^{4} \\
\leq & 4\left\|\boldsymbol{\theta}-\boldsymbol{\theta}_{0}\right\|^{4}+o(1) .
\end{aligned}
$$

Due to Condition $(\mathrm{C} 2)$, we have $\operatorname{var}\left(\alpha_{i}\right)=o(1)$ and then $\alpha_{i}=o_{p}(1)$. Thus,

$$
\begin{aligned}
& \mathbb{E}\left(A_{1}^{2}\right) \\
= & \frac{4}{n^{2}(n-1)^{2}} \mathbb{E}\left(\sum_{i<j}\left[\omega\left\{v_{i}\left(1+\alpha_{i}\right)^{1 / 2}\right\} \omega\left\{v_{j}\left(1+\alpha_{j}\right)^{1 / 2}\right\}-\omega\left(v_{i}\right) \omega\left(v_{j}\right)\right]\right. \\
& \left.\times \boldsymbol{S}_{i}^{\mathrm{T}} \boldsymbol{S}_{j}\right)^{2} \\
= & \frac{4}{n^{2}(n-1)^{2}} \mathbb{E}\left\{\sum _ { i < j } \left(\left[\omega\left(v_{i}\right)^{-1} \omega\left\{v_{i}\left(1+\alpha_{i}\right)^{1 / 2}\right\} \omega\left(v_{j}\right)^{-1} \omega\left\{v_{j}\left(1+\alpha_{j}\right)^{1 / 2}\right\}\right.\right.\right. \\
& \left.\left.-1] \times \boldsymbol{V}_{i}^{\mathrm{T}} \boldsymbol{V}_{j}\right)^{2}\right\} \\
= & O\left(n^{-2}\right) \mathbb{E}\left(\left[\omega\left(v_{i}\right)^{-1} \omega\left\{v_{i}\left(1+\alpha_{i}\right)^{1 / 2}\right\} \omega\left(v_{j}\right)^{-1} \omega\left\{v_{j}\left(1+\alpha_{j}\right)^{1 / 2}\right\}-1\right]\right. \\
& \left.\times \boldsymbol{V}_{i}^{\mathrm{T}} \boldsymbol{V}_{j}\right)^{2}
\end{aligned}
$$




$$
\begin{aligned}
\leq & O\left(n^{-2}\right) \sqrt{\mathbb{E}\left(\left[\omega\left(v_{i}\right)^{-1} \omega\left\{v_{i}\left(1+\alpha_{i}\right)^{1 / 2}\right\} \omega\left(v_{j}\right)^{-1} \omega\left\{v_{j}\left(1+\alpha_{j}\right)^{1 / 2}\right\}-1\right]^{4}\right)} \\
& \times \sqrt{\mathbb{E}\left\{\left(\boldsymbol{V}_{i}^{\mathrm{T}} \boldsymbol{V}_{j}\right)^{4}\right\}} \\
= & O\left\{n^{-2} b_{4}(\omega)\right\} \sqrt{\mathbb{E}\left\{\left(\boldsymbol{S}_{i}^{\mathrm{T}} \boldsymbol{S}_{j}\right)^{4}\right\}} \\
= & O\left\{\sigma_{n}^{2}(\omega)\right\}
\end{aligned}
$$

where the second last equality is due to $\alpha_{i} \rightarrow 0$ and Lemma A.1 (iii). This leads to $A_{1}=o_{p}\left\{\sigma_{n}(\omega)\right\}$. Next, for the first term in $A_{2}$, we have

$$
\begin{aligned}
\mathbb{E}\left(A_{21}^{2}\right)=\mathbb{E} & \left(\left[\frac{2}{n(n-1)} \sum_{i<j} \sum_{j} \omega\left\{v_{i}\left(1+\alpha_{i}\right)^{1 / 2}\right\} \omega\left\{v_{j}\left(1+\alpha_{j}\right)^{1 / 2}\right\} v_{i}^{-1}\right.\right. \\
& \left.\left.\times \boldsymbol{X}_{i}^{\mathrm{T}}\left(\boldsymbol{\theta} \boldsymbol{\theta}^{\mathrm{T}}-\boldsymbol{\theta}_{0} \boldsymbol{\theta}_{0}^{\mathrm{T}}\right) \boldsymbol{S}_{j}\right]^{2}\right) \\
= & O\left(n^{-4}\right) \mathbb{E}\left(\left[\sum_{i<j} \sum_{i} \omega\left\{v_{i}\left(1+\alpha_{i}\right)^{1 / 2}\right\} \omega\left\{v_{j}\left(1+\alpha_{j}\right)^{1 / 2}\right\} v_{i}^{-1}\right.\right. \\
& \left.\left.\times \boldsymbol{X}_{i}^{\mathrm{T}}\left(\boldsymbol{\theta} \boldsymbol{\theta}^{\mathrm{T}}-\boldsymbol{\theta}_{0} \boldsymbol{\theta}_{0}^{\mathrm{T}}\right) \boldsymbol{S}_{j}\right]^{2}\right) \\
= & O\left(n^{-4}\right) \mathbb{E}\left(\left[\sum_{i<j} \sum \omega\left\{v_{i}\left(1+\alpha_{i}\right)^{1 / 2}\right\} \omega\left\{v_{j}\left(1+\alpha_{j}\right)^{1 / 2}\right\} v_{i}^{-1}\right.\right. \\
& \left.\left.\times \boldsymbol{X}_{i}^{\mathrm{T}} \boldsymbol{\theta}_{0} \boldsymbol{\theta}_{0}^{\mathrm{T}} \boldsymbol{S}_{j}\right]^{2}\right) \\
\doteq & O\left(n^{-2}\right) \mathbb{E}\left\{\left[\omega\left(v_{i}\right) \omega\left(v_{j}\right) v_{i}^{-1}\left(u_{i} \boldsymbol{\theta}^{\mathrm{T}} \boldsymbol{\theta}_{0} \boldsymbol{\theta}_{0}^{\mathrm{T}} \boldsymbol{S}_{j}+v_{i} \boldsymbol{S}_{i}^{\mathrm{T}} \boldsymbol{\theta}_{0} \boldsymbol{\theta}_{0}^{\mathrm{T}} \boldsymbol{S}_{j}\right)\right]^{2}\right\} \\
+ & O\left(n^{-1}\right) \mathbb{E}\left\{\omega\left(v_{i_{1}}\right) \omega\left(v_{i_{2}}\right) \omega\left(v_{j}\right)^{2} v_{i_{1}}^{-1} v_{i_{2}}^{-1} u_{i_{1}} u_{i_{2}}\right\} \\
& \times \mathbb{E}\left(\boldsymbol{\theta}^{\mathrm{T}} \boldsymbol{\theta}_{0} \boldsymbol{\theta}_{0}^{\mathrm{T}} \boldsymbol{S}_{j} \boldsymbol{S}_{j}^{\mathrm{T}} \boldsymbol{\theta}_{0} \boldsymbol{\theta}_{0}^{\mathrm{T}} \boldsymbol{\theta}\right),
\end{aligned}
$$

where $i_{1} \neq i_{2}<j$. We have that

$$
\begin{aligned}
& O\left(n^{-2}\right) \mathbb{E}\left[\left\{\omega\left(v_{i}\right) \omega\left(v_{j}\right) v_{i}^{-1}\left(u_{i} \boldsymbol{\theta}^{\mathrm{T}} \boldsymbol{\theta}_{0} \boldsymbol{\theta}_{0}^{\mathrm{T}} \boldsymbol{S}_{j}+v_{i} \boldsymbol{S}_{i}^{\mathrm{T}} \boldsymbol{\theta}_{0} \boldsymbol{\theta}_{0}^{\mathrm{T}} \boldsymbol{S}_{j}\right)\right\}^{2}\right] \\
& \leq O\left(n^{-2}\right) \mathbb{E}\left\{\omega\left(v_{i}\right) \omega\left(v_{j}\right) v_{i}^{-1} u_{i} \boldsymbol{\theta}^{\mathrm{T}} \boldsymbol{\theta}_{0} \boldsymbol{\theta}_{0}^{\mathrm{T}} \boldsymbol{S}_{j}\right\}^{2}+O\left(n^{-2}\right) \mathbb{E}\left\{\omega\left(v_{i}\right) \omega\left(v_{j}\right) \boldsymbol{S}_{i}^{\mathrm{T}} \boldsymbol{\theta}_{0} \boldsymbol{\theta}_{0}^{\mathrm{T}} \boldsymbol{S}_{j}\right\}^{2} \\
& =O\left(n^{-2}\right) \mathbb{E}\left\{\omega^{2}\left(v_{i}\right) \omega^{2}\left(v_{j}\right) v_{i}^{-2} u_{i}^{2}\right\} \mathbb{E}\left\{\left(\boldsymbol{\theta}^{\mathrm{T}} \boldsymbol{\theta}_{0} \boldsymbol{\theta}_{0}^{\mathrm{T}} \boldsymbol{S}_{j}\right)^{2}\right\} \\
& +O\left\{n^{-2} b_{2}^{2}(\omega)\right\} \mathbb{E}\left\{\left(\boldsymbol{S}_{i}^{\mathrm{T}} \boldsymbol{\theta}_{0} \boldsymbol{\theta}_{0}^{\mathrm{T}} \boldsymbol{S}_{j}\right)^{2}\right\} \\
& \leq O\left(n^{-2}\right) \mathbb{E}\left\{\omega^{2}\left(v_{j}\right)\right\} \sqrt{\mathbb{E}\left(v_{i}^{-4} u_{i}^{4}\right) \mathbb{E}\left\{\omega^{4}\left(v_{i}\right)\right\}} \mathbb{E}\left(\boldsymbol{\theta}^{\mathrm{T}} \boldsymbol{\theta}_{0} \boldsymbol{\theta}_{0}^{\mathrm{T}} \boldsymbol{S}_{j} \boldsymbol{S}_{j}^{\mathrm{T}} \boldsymbol{\theta}_{0} \boldsymbol{\theta}_{0}^{\mathrm{T}} \boldsymbol{\theta}\right) \\
& +O\left\{n^{-2} b_{2}^{2}(\omega)\right\} \mathbb{E}\left(\boldsymbol{S}_{i}^{\mathrm{T}} \boldsymbol{\theta}_{0} \boldsymbol{\theta}_{0}^{\mathrm{T}} \boldsymbol{S}_{j} \boldsymbol{S}_{j}^{\mathrm{T}} \boldsymbol{\theta}_{0} \boldsymbol{\theta}_{0}^{\mathrm{T}} \boldsymbol{S}_{i}\right) \\
& =o\left\{\sigma_{n}^{2}(\omega)\right\} \text {, }
\end{aligned}
$$

where the second last equality is due to Lemma A.1 (i) and Condition (C2).

Then, due to $i_{1} \neq i_{2}<j$, we have that

$$
O\left(n^{-1}\right) \mathbb{E}\left\{\omega\left(v_{i_{1}}\right) \omega\left(v_{i_{2}}\right) \omega\left(v_{j}\right)^{2} v_{i_{1}}^{-1} v_{i_{2}}^{-1} u_{i_{1}} u_{i_{2}}\right\} \mathbb{E}\left(\boldsymbol{\theta}^{\mathrm{T}} \boldsymbol{\theta}_{0} \boldsymbol{\theta}_{0}^{\mathrm{T}} \boldsymbol{S}_{j} \boldsymbol{S}_{j}^{\mathrm{T}} \boldsymbol{\theta}_{0} \boldsymbol{\theta}_{0}^{\mathrm{T}} \boldsymbol{\theta}\right)
$$




$$
\begin{aligned}
& =O\left(n^{-1}\right) b_{2}(\omega)\left[\mathbb{E}\left\{\omega\left(v_{i}\right) v_{i}^{-1} u_{i}\right\}\right]^{2} \mathbb{E}\left(\boldsymbol{\theta}^{\mathrm{T}} \boldsymbol{\theta}_{0} \boldsymbol{\theta}_{0}^{\mathrm{T}} \boldsymbol{S}_{j} \boldsymbol{S}_{j}^{\mathrm{T}} \boldsymbol{\theta}_{0} \boldsymbol{\theta}_{0}^{\mathrm{T}} \boldsymbol{\theta}\right) \\
& \leq O\left\{n^{-1}(p-1)^{-1}\right\} b_{2}(\omega) \mathbb{E}\left\{\omega^{2}\left(v_{i}\right) v_{i}^{-2} u_{i}^{2}\right\}\left\{\boldsymbol{\theta}^{\mathrm{T}} \boldsymbol{\theta}_{0} \boldsymbol{\theta}_{0}^{\mathrm{T}} \boldsymbol{\theta}-\left(\boldsymbol{\theta}^{\mathrm{T}} \boldsymbol{\theta}_{0} \boldsymbol{\theta}_{0}^{\mathrm{T}} \boldsymbol{\theta}\right)^{2}\right\} \\
& \leq O\left\{n^{-1}(p-1)^{-1} b_{2}(\omega)\right\} \sqrt{\mathbb{E}\left\{\omega^{4}\left(v_{i}\right)\right\} \mathbb{E}\left(v_{i}^{-4} u_{i}^{4}\right)}\left\{\boldsymbol{\theta}^{\mathrm{T}} \boldsymbol{\theta}_{0} \boldsymbol{\theta}_{0}^{\mathrm{T}} \boldsymbol{\theta}-\left(\boldsymbol{\theta}^{\mathrm{T}} \boldsymbol{\theta}_{0} \boldsymbol{\theta}_{0}^{\mathrm{T}} \boldsymbol{\theta}\right)^{2}\right\} \\
& =O\left\{n^{-1}(p-1)^{-1} b_{2}^{2}(\omega)\right\} \sqrt{\mathbb{E}\left(v_{i}^{-4} u_{i}^{4}\right)}\left\{\boldsymbol{\theta}^{\mathrm{T}} \boldsymbol{\theta}_{0} \boldsymbol{\theta}_{0}^{\mathrm{T}} \boldsymbol{\theta}-\left(\boldsymbol{\theta}^{\mathrm{T}} \boldsymbol{\theta}_{0} \boldsymbol{\theta}_{0}^{\mathrm{T}} \boldsymbol{\theta}\right)^{2}\right\} \\
& =o\left\{\sigma_{n}^{2}(\omega)\right\} \text {, }
\end{aligned}
$$

where $i \neq j$ in the first equality, and the second last equality is due to Condition (C1). In addition,

$$
\begin{aligned}
& \left\{\boldsymbol{\theta}^{\mathrm{T}} \boldsymbol{\theta}_{0} \boldsymbol{\theta}_{0}^{\mathrm{T}} \boldsymbol{\theta}-\left(\boldsymbol{\theta}^{\mathrm{T}} \boldsymbol{\theta}_{0} \boldsymbol{\theta}_{0}^{\mathrm{T}} \boldsymbol{\theta}\right)^{2}\right\} \\
= & \left(\boldsymbol{\theta}^{\mathrm{T}} \boldsymbol{\theta}_{0}\right)^{2}\left(\left\|\boldsymbol{\theta}-\boldsymbol{\theta}_{0}\right\|^{2}-\left\|\boldsymbol{\theta}-\boldsymbol{\theta}_{0}\right\|^{4} / 4\right),
\end{aligned}
$$

hence the last equation holds and $A_{21}=o_{p}\left\{\sigma_{n}(\omega)\right\}$. Similarly, $A_{22}=o_{p}\left\{\sigma_{n}(\omega)\right\}$, hence $A_{2}=o_{p}\left\{\sigma_{n}(\omega)\right\}$. Next, define $\beta_{i} \dot{=} \omega\left\{v_{i}\left(1+\alpha_{i}\right)^{1 / 2}\right\} v_{i}^{-1}$, and $\beta_{0} \doteq \mathbb{E}\left(\beta_{i}\right)$. Then $\beta_{0}=c_{0}(\omega)\{1+o(1)\}$.

$$
\begin{aligned}
\mathbb{E}\left(\beta_{i}^{2}\right) & =\mathbb{E}\left\{\omega^{2}\left(v_{i}\right) v_{i}^{-2}\right\}\{1+o(1)\} \\
& \leq\left[\mathbb{E}\left\{\omega^{4}\left(v_{i}\right)\right\} \mathbb{E}\left(v_{i}^{-4}\right)\right]^{1 / 2} \\
& \leq O\left\{b_{2}(\omega)\right\} \sqrt{\mathbb{E}\left(v_{i}^{-4}\right)} .
\end{aligned}
$$

Note that $\beta_{0}^{2} \leq \mathbb{E}\left(\beta_{i}^{2}\right)$. We let

$$
\begin{aligned}
A_{3}^{\prime}= & \frac{2}{n(n-1)} \sum_{i<j} \omega\left\{v_{i}\left(1+\alpha_{i}\right)^{1 / 2}\right\} \omega\left\{v_{j}\left(1+\alpha_{j}\right)^{1 / 2}\right\} v_{i}^{-1} v_{j}^{-1} \\
& \left\{\mathbb{E}\left(u_{i} u_{j}\right) \boldsymbol{\theta}^{\mathrm{T}}\left(\boldsymbol{\theta} \boldsymbol{\theta}^{\mathrm{T}}-\boldsymbol{\theta}_{0} \boldsymbol{\theta}_{0}^{\mathrm{T}}\right) \boldsymbol{\theta}+v_{i} v_{j} \boldsymbol{S}_{i}^{\mathrm{T}} \boldsymbol{\theta}_{0} \boldsymbol{\theta}_{0}^{\mathrm{T}} \boldsymbol{S}_{j}\right\} .
\end{aligned}
$$

Next, we will show $A_{3}=A_{3}^{\prime}+o_{p}\left\{\sigma_{n}(\omega)\right\}$ i.e. $A_{3}-A_{3}^{\prime}=o_{p}\left\{\sigma_{n}(\omega)\right\}$.

$$
\begin{aligned}
\mathbb{E}\left(A_{3}-A_{3}^{\prime}\right)= & \mathbb{E}\left[\frac{2}{n(n-1)} \sum_{i<j} \sum_{j} \omega\left\{v_{i}\left(1+\alpha_{i}\right)^{1 / 2}\right\} v_{i}^{-1} \omega\left\{v_{j}\left(1+\alpha_{j}\right)^{1 / 2}\right\} v_{j}^{-1}\right. \\
& \left.\times\left\{u_{i} u_{j}-\mathbb{E}^{2}\left(u_{i}\right)\right\} \boldsymbol{\theta}^{\mathrm{T}}\left(\boldsymbol{\theta} \boldsymbol{\theta}^{\mathrm{T}}-\boldsymbol{\theta}_{0} \boldsymbol{\theta}_{0}^{\mathrm{T}}\right) \boldsymbol{\theta}\right] \\
= & \boldsymbol{\theta}^{\mathrm{T}}\left(\boldsymbol{\theta} \boldsymbol{\theta}^{\mathrm{T}}-\boldsymbol{\theta}_{0} \boldsymbol{\theta}_{0}^{\mathrm{T}}\right) \boldsymbol{\theta} \mathbb{E}\left[\omega\left\{v_{i}\left(1+\alpha_{i}\right)^{1 / 2}\right\} v_{i}^{-1} \omega\left\{v_{j}\left(1+\alpha_{j}\right)^{1 / 2}\right\}\right. \\
& \left.\times v_{j}^{-1}\left\{u_{i} u_{j}-\mathbb{E}^{2}\left(u_{i}\right)\right\}\right] \\
= & \boldsymbol{\theta}^{\mathrm{T}}\left(\boldsymbol{\theta} \boldsymbol{\theta}^{\mathrm{T}}-\boldsymbol{\theta}_{0} \boldsymbol{\theta}_{0}^{\mathrm{T}}\right) \boldsymbol{\theta} \sqrt{\mathbb{E}\left(\beta_{i}^{2} \beta_{j}^{2}\right) \mathbb{E}\left(u_{i} u_{j}-\mathbb{E}^{2}\left(u_{i}\right)\right)^{2}} \\
\leq & \boldsymbol{\theta}^{\mathrm{T}}\left(\boldsymbol{\theta} \boldsymbol{\theta}^{\mathrm{T}}-\boldsymbol{\theta}_{0} \boldsymbol{\theta}_{0}^{\mathrm{T}}\right) \boldsymbol{\theta} \mathbb{E}\left(\beta_{i}^{2}\right) \sqrt{\mathbb{E}^{2}\left(u_{i}^{2}\right)-\mathbb{E}^{4}\left(u_{i}\right)} \\
\leq & \boldsymbol{\theta}^{\mathrm{T}}\left(\boldsymbol{\theta} \boldsymbol{\theta}^{\mathrm{T}}-\boldsymbol{\theta}_{0} \boldsymbol{\theta}_{0}^{\mathrm{T}}\right) \boldsymbol{\theta} \sqrt{b_{4}(\omega) \mathbb{E}\left(v_{i}^{-4}\right)} \sqrt{\mathbb{E}^{2}\left(u_{i}^{2}\right)-\mathbb{E}^{4}\left(u_{i}\right)} \\
= & o\left\{\sigma_{n}(\omega)\right\},
\end{aligned}
$$


where the last equality is due to Condition (C2). Hence we have

$$
\mathbb{E}^{2}\left(u_{i}^{2}\right)-\mathbb{E}^{4}\left(u_{i}\right)=o(1)
$$

So, we define $u_{i} u_{j}-\mathbb{E}^{2}\left(u_{i}\right)=\gamma_{i j}$, then

$$
\begin{aligned}
\operatorname{var}\left(A_{3}-A_{3}^{\prime}\right)= & \mathbb{E}\left\{\frac{2}{n(n-1)} \sum_{i<j} \sum_{i} \beta_{i} \beta_{j} \gamma_{i j} \boldsymbol{\theta}^{\mathrm{T}}\left(\boldsymbol{\theta} \boldsymbol{\theta}^{\mathrm{T}}-\boldsymbol{\theta}_{0} \boldsymbol{\theta}_{0}^{\mathrm{T}}\right) \boldsymbol{\theta}\right\}^{2} \\
& -\mathbb{E}^{2}\left\{\frac{2}{n(n-1)} \beta_{i} \beta_{j} \gamma_{i j} \boldsymbol{\theta}^{\mathrm{T}}\left(\boldsymbol{\theta} \boldsymbol{\theta}^{\mathrm{T}}-\boldsymbol{\theta}_{0} \boldsymbol{\theta}_{0}^{\mathrm{T}}\right) \boldsymbol{\theta}\right\} \\
= & \left\{\boldsymbol{\theta}^{\mathrm{T}}\left(\boldsymbol{\theta} \boldsymbol{\theta}^{\mathrm{T}}-\boldsymbol{\theta}_{0} \boldsymbol{\theta}_{0}^{\mathrm{T}}\right) \boldsymbol{\theta}\right\}^{2}\left\{O\left(n^{-1}\right) \mathbb{E}\left(\beta_{i}^{2} \beta_{j} \beta_{l} \gamma_{i j} \gamma_{i l}\right)\right. \\
& \left.+O\left(n^{-2}\right) \mathbb{E}\left(\beta_{i}^{2} \beta_{j}^{2} \gamma_{i j}^{2}\right)\right\} \\
= & D_{1}+D_{2},
\end{aligned}
$$

where $i<j \neq l$ in the second inequality. At first, we focus on $D_{1}$. Due to $i<j \neq l$,

$$
\begin{aligned}
D_{1}= & \left\{\boldsymbol{\theta}^{\mathrm{T}}\left(\boldsymbol{\theta} \boldsymbol{\theta}^{\mathrm{T}}-\boldsymbol{\theta}_{0} \boldsymbol{\theta}_{0}^{\mathrm{T}}\right) \boldsymbol{\theta}\right\}^{2} O\left(n^{-1}\right) \mathbb{E}\left(\beta_{i}^{2} \beta_{j} \beta_{l} \gamma_{i j} \gamma_{i l}\right) \\
= & \left\{\boldsymbol{\theta}^{\mathrm{T}}\left(\boldsymbol{\theta} \boldsymbol{\theta}^{\mathrm{T}}-\boldsymbol{\theta}_{0} \boldsymbol{\theta}_{0}^{\mathrm{T}}\right) \boldsymbol{\theta}\right\}^{2} O\left(n^{-1}\right)\left[\mathbb{E}\left(\beta_{i}^{2} \beta_{j} \beta_{l} u_{i}^{2} u_{j} u_{l}\right)-\mathbb{E}\left\{\beta_{i}^{2} \beta_{j} \beta_{l} \mathbb{E}^{2}\left(u_{i}\right) u_{i} u_{j}\right\}\right. \\
& \left.-\mathbb{E}\left\{\beta_{i}^{2} \beta_{j} \beta_{l} \mathbb{E}^{2}\left(u_{i}\right) u_{i} u_{l}\right\}+\mathbb{E}\left\{\beta_{i}^{2} \beta_{j} \beta_{l} \mathbb{E}^{4}\left(u_{i}\right)\right\}\right] \\
\leq & \left\{\boldsymbol{\theta}^{\mathrm{T}}\left(\boldsymbol{\theta} \boldsymbol{\theta}^{\mathrm{T}}-\boldsymbol{\theta}_{0} \boldsymbol{\theta}_{0}^{\mathrm{T}}\right) \boldsymbol{\theta}\right\}^{2} O\left(n^{-1}\right) b_{4}(\omega) \mathbb{E}\left(v_{i}^{-4}\right) \\
= & O\left\{n^{-1} b_{2}^{2}(\omega)\right\}\left\{\boldsymbol{\theta}^{\mathrm{T}}\left(\boldsymbol{\theta} \boldsymbol{\theta}^{\mathrm{T}}-\boldsymbol{\theta}_{0} \boldsymbol{\theta}_{0}^{\mathrm{T}}\right) \boldsymbol{\theta}\right\}^{2} \mathbb{E}\left(v_{i}^{-4}\right) \\
= & o\left\{\sigma_{n}^{2}(\omega)\right\}
\end{aligned}
$$

where the first inequality is because of the Cauchy inequality and $\left|u_{i}\right| \leq 1$. The Condition ( $\mathrm{C} 2)$ brings about the last equality. Next, similar to the above process, we have

$$
\begin{aligned}
D_{2} & =\left\{\boldsymbol{\theta}^{\mathrm{T}}\left(\boldsymbol{\theta} \boldsymbol{\theta}^{\mathrm{T}}-\boldsymbol{\theta}_{0} \boldsymbol{\theta}_{0}^{\mathrm{T}}\right) \boldsymbol{\theta}\right\}^{2} O\left(n^{-2}\right) \mathbb{E}\left[\beta_{i}^{2} \beta_{j}^{2}\left\{u_{i}^{2} u_{j}^{2}+\mathbb{E}^{4}\left(u_{i}\right)-2 u_{i} u_{j} \mathbb{E}^{2}\left(u_{i}\right)\right\}\right] \\
& \leq O\left(n^{-2}\right)\left\{\boldsymbol{\theta}^{\mathrm{T}}\left(\boldsymbol{\theta} \boldsymbol{\theta}^{\mathrm{T}}-\boldsymbol{\theta}_{0} \boldsymbol{\theta}_{0}^{\mathrm{T}}\right) \boldsymbol{\theta}\right\}^{2} b_{4}(\omega) \mathbb{E}\left(v_{i}^{-4}\right) \\
& \leq O\left\{n^{-2} b_{2}^{2}(\omega)\right\}\left\{\boldsymbol{\theta}^{\mathrm{T}}\left(\boldsymbol{\theta} \boldsymbol{\theta}^{\mathrm{T}}-\boldsymbol{\theta}_{0} \boldsymbol{\theta}_{0}^{\mathrm{T}}\right) \boldsymbol{\theta}\right\}^{2} \mathbb{E}\left(v_{i}^{-4}\right) \\
& =o\left\{\sigma_{n}^{2}(\omega)\right\} .
\end{aligned}
$$

Therefore, we can conclude that $A_{3}=A_{3}^{\prime}+o_{p}\left\{\sigma_{n}(\omega)\right\}$. Then, for $A_{3}^{\prime}$, we have

$$
\begin{aligned}
\mathbb{E}\left(A_{3}^{\prime}\right) & =\beta_{0}^{2} \mathbb{E}^{2}\left(u_{i}\right) \boldsymbol{\theta}^{\mathrm{T}}\left(\boldsymbol{\theta} \boldsymbol{\theta}^{\mathrm{T}}-\boldsymbol{\theta}_{0} \boldsymbol{\theta}_{0}^{\mathrm{T}}\right) \boldsymbol{\theta} \\
& =c_{0}^{2}(\omega) \mathbb{E}^{2}\left(u_{i}\right) \boldsymbol{\theta}^{\mathrm{T}}\left(\boldsymbol{\theta} \boldsymbol{\theta}^{\mathrm{T}}-\boldsymbol{\theta}_{0} \boldsymbol{\theta}_{0}^{\mathrm{T}}\right) \boldsymbol{\theta}\{1+o(1)\} .
\end{aligned}
$$

Moreover,

$$
\operatorname{var}\left(A_{3}^{\prime}\right)=\mathbb{E}\left(\frac { 2 } { n ( n - 1 ) } \sum _ { i < j } \sum _ { i } \left[\beta_{i} \beta_{j} \mathbb{E}^{2}\left(u_{i}\right)\left\{\boldsymbol{\theta}^{\mathrm{T}}\left(\boldsymbol{\theta} \boldsymbol{\theta}^{\mathrm{T}}-\boldsymbol{\theta}_{0} \boldsymbol{\theta}_{0}^{\mathrm{T}}\right) \boldsymbol{\theta}\right\}\right.\right.
$$




$$
\begin{aligned}
& \left.\left.+\omega\left(v_{i}\right) \omega\left(v_{j}\right) \boldsymbol{S}_{i}^{\mathrm{T}} \boldsymbol{\theta}_{0} \boldsymbol{\theta}_{0}^{\mathrm{T}} \boldsymbol{S}_{j}\right]\right)^{2}-\beta_{0}^{4} \mathbb{E}^{4}\left(u_{i}\right)\left\{\boldsymbol{\theta}^{\mathrm{T}}\left(\boldsymbol{\theta} \boldsymbol{\theta}^{\mathrm{T}}-\boldsymbol{\theta}_{0} \boldsymbol{\theta}_{0}^{\mathrm{T}}\right) \boldsymbol{\theta}\right\}^{2} \\
= & \mathbb{E}\left\{\frac{2}{n(n-1)} \sum_{i<j} \sum_{i} \beta_{i} \beta_{j} \mathbb{E}^{2}\left(u_{i}\right) \boldsymbol{\theta}^{\mathrm{T}}\left(\boldsymbol{\theta} \boldsymbol{\theta}^{\mathrm{T}}-\boldsymbol{\theta}_{0} \boldsymbol{\theta}_{0}^{\mathrm{T}}\right) \boldsymbol{\theta}\right\}^{2} \\
& +\mathbb{E}\left\{\frac{2}{n(n-1)} \sum_{i<j} \sum_{i} \omega\left(v_{i}\right)\left(1+\alpha_{i}\right)^{1 / 2} \omega\left(v_{j}\right)\left(1+\alpha_{j}\right)^{1 / 2} \boldsymbol{S}_{i}^{\mathrm{T}} \boldsymbol{\theta}_{0} \boldsymbol{\theta}_{0}^{\mathrm{T}} \boldsymbol{S}_{j}\right\}^{2} \\
& -\beta_{0}^{4} \mathbb{E}^{4}\left(u_{i}\right)\left\{\boldsymbol{\theta}^{\mathrm{T}}\left(\boldsymbol{\theta} \boldsymbol{\theta}^{\mathrm{T}}-\boldsymbol{\theta}_{0} \boldsymbol{\theta}_{0}^{\mathrm{T}}\right) \boldsymbol{\theta}\right\}^{2} \\
= & O\left\{n^{-4}\left(\boldsymbol{\theta}^{\mathrm{T}}\left(\boldsymbol{\theta} \boldsymbol{\theta}^{\mathrm{T}}-\boldsymbol{\theta}_{0} \boldsymbol{\theta}_{0}^{\mathrm{T}}\right) \boldsymbol{\theta}\right)^{2}\right\} \mathbb{E}\left\{\sum \sum_{i<j} \sum_{k<l} \sum_{i}\left(\beta_{i} \beta_{j} \beta_{k} \beta_{l}-\beta_{0}^{4}\right)\right\} \\
& +O\left\{n^{-2} b_{2}^{2}(\omega)\right\} \mathbb{E}\left(\boldsymbol{S}_{i}^{\mathrm{T}} \boldsymbol{\theta}_{0} \boldsymbol{\theta}_{0}^{\mathrm{T}} \boldsymbol{S}_{j} \boldsymbol{S}_{j}^{\mathrm{T}} \boldsymbol{\theta}_{0} \boldsymbol{\theta}_{0}^{\mathrm{T}} \boldsymbol{S}_{i}\right) \\
= & \left(\boldsymbol{\theta}^{\mathrm{T}}\left(\boldsymbol{\theta} \boldsymbol{\theta}^{\mathrm{T}}-\boldsymbol{\theta}_{0} \boldsymbol{\theta}_{0}^{\mathrm{T}}\right) \boldsymbol{\theta}\right)^{2}\left\{O\left(n^{-2}\right) \mathbb{E}\left(\beta_{i}^{2} \beta_{j}^{2}-\beta_{0}^{4}\right)\right. \\
& \left.+O\left(n^{-1}\right) \beta_{0}^{2} \mathbb{E}\left(\beta_{i}^{2}-\beta_{0}^{2}\right)\right\}+o\left(n^{-2} b_{2}^{2}(\omega) p^{-2}\right) \\
\leq & \left(\boldsymbol{\theta}^{\mathrm{T}}\left(\boldsymbol{\theta} \boldsymbol{\theta}^{\mathrm{T}}-\boldsymbol{\theta}_{0} \boldsymbol{\theta}_{0}^{\mathrm{T}}\right) \boldsymbol{\theta}\right)^{2}\left[O\left(n^{-2}\right)\left\{\mathbb{E}\left(\beta_{i}^{2}\right)\right\}^{2}+O\left(n^{-1}\right)\left\{\mathbb{E}\left(\beta_{i}^{2}\right)\right\}^{2}\right] \\
& +o\left\{n^{-2} b_{2}^{2}(\omega) p^{-2}\right\} \\
= & \left(\boldsymbol{\theta}^{\mathrm{T}}\left(\boldsymbol{\theta} \boldsymbol{\theta}^{\mathrm{T}}-\boldsymbol{\theta}_{0} \boldsymbol{\theta}_{0}^{\mathrm{T}}\right) \boldsymbol{\theta}\right)^{2}\left\{O\left(n^{-1}\right) b_{2}^{2}(\omega) \mathbb{E}\left(v_{i}^{-4}\right)\right\}+o\left\{\sigma_{n}^{2}(\omega)\right\} \\
= & o\left\{\sigma_{n}^{2}(\omega)\right\},
\end{aligned}
$$

where the last equality is due to Condition $(\mathrm{C} 2)$, which leads to

$$
A_{3}=c_{0}(\omega)^{2} \mathbb{E}^{2}\left(u_{i}\right)\left\{\boldsymbol{\theta}^{\mathrm{T}}\left(\boldsymbol{\theta} \boldsymbol{\theta}^{\mathrm{T}}-\boldsymbol{\theta}_{0} \boldsymbol{\theta}_{0}^{\mathrm{T}}\right) \boldsymbol{\theta}\right\}+o_{p}\left\{\sigma_{n}(\omega)\right\} .
$$

For $A_{4}$, by the Cauchy inequality, we have

$$
\begin{aligned}
\mathbb{E}\left(A_{4}^{2}\right)= & \frac{4}{n^{2}(n-1)^{2}} \sum_{i<j} \sum_{i} \mathbb{E}\left[\boldsymbol{V}_{i}^{\mathrm{T}} \boldsymbol{V}_{j}\left\{\left(1+\alpha_{i}\right)^{-1 / 2}\left(1+\alpha_{j}\right)^{-1 / 2}-1\right\}\right]^{2} \\
& \times\{1+o(1)\} \\
= & O\left(n^{-2}\right) \mathbb{E}\left\{\left(\boldsymbol{V}_{i}^{\mathrm{T}} \boldsymbol{V}_{j}\right)^{2}\right\} \mathbb{E}\left[\left\{\left(1+\alpha_{i}\right)^{-1 / 2}\left(1+\alpha_{j}\right)^{-1 / 2}-1\right\}^{2}\right] \\
& \times\{1+o(1)\} \\
= & o\left\{n^{-2} b_{2}^{2}(\omega)(p-1)^{-1}\right\} \\
= & o\left\{n^{-2} p^{-1} b_{2}^{2}(\omega)\right\} \\
= & o\left\{\sigma_{n}^{2}(\omega)\right\}
\end{aligned}
$$

hence $A_{4}=o_{p}\left\{\sigma_{n}(\omega)\right\}$. Similarly, we can obtain that $\mathbb{E}\left(A_{5}^{2}\right)=o\left\{\mathbb{E}\left(A_{2}^{2}\right)\right\}$ and $\mathbb{E}\left(A_{6}^{2}\right)=o\left\{\mathbb{E}\left(A_{3}^{2}\right)\right\}$, which lead to $A_{5}=o_{p}\left\{\sigma_{n}(\omega)\right\}, A_{6}=o_{p}\left\{\sigma_{n}(\omega)\right\}$, respectively.

Combining the above results, we get

$$
T_{n}(\omega)=\frac{2}{n(n-1)} \sum_{i<j} \sum_{i} \boldsymbol{V}_{i}^{\mathrm{T}} \boldsymbol{V}_{j}+c_{0}(\omega)^{2}\left\{\boldsymbol{\theta}^{\mathrm{T}}\left(\boldsymbol{\theta} \boldsymbol{\theta}^{\mathrm{T}}-\boldsymbol{\theta}_{0} \boldsymbol{\theta}_{0}^{\mathrm{T}}\right) \boldsymbol{\theta}\right\}+o_{p}\left\{\sigma_{n}(\omega)\right\},
$$

using the same procedure as in the proof of Theorem 1 . Then, we obtain the conclusion. 


\section{Proof of theorem 3.4}

Under conditions $(\mathrm{C} 1)-(\mathrm{C} 2)$, given $\boldsymbol{\theta}$ and $\boldsymbol{\theta}_{0}$, we find that the right side of (3.1) is an increasing function of $\mathbb{E}^{2}\left\{\omega\left(v_{i}\right) v_{i}^{-1}\right\} / \mathbb{E}\left\{\omega^{2}\left(v_{i}\right)\right\}$. Interestingly, according to the Cauchy inequality, it can be seen that

$$
\frac{\mathbb{E}^{2}\left\{\omega\left(v_{i}\right) v_{i}^{-1}\right\}}{\mathbb{E}\left\{\omega^{2}\left(v_{i}\right)\right\}} \leq \frac{\mathbb{E}\left\{\omega^{2}\left(v_{i}\right)\right\} \mathbb{E}\left(v_{i}^{-2}\right)}{\mathbb{E}\left\{\omega^{2}\left(v_{i}\right)\right\}}=\mathbb{E}\left(v_{i}^{-2}\right) .
$$

The above inequality holds for any nonnegative continuous weight function satisfying the condition (C1). So, the maximum value of the asymptotic power in the whole family is

$$
\lim _{n, p \rightarrow \infty} \Phi\left[-z_{\alpha}+\mathbb{E}^{2}\left(u_{i}\right) \mathbb{E}\left(v_{i}{ }^{-2}\right) \frac{p^{1 / 2} n\left\{\boldsymbol{\theta}^{\mathrm{T}}\left(\boldsymbol{\theta} \boldsymbol{\theta}^{\mathrm{T}}-\boldsymbol{\theta}_{0} \boldsymbol{\theta}_{0}^{\mathrm{T}}\right) \boldsymbol{\theta}\right\}}{\sqrt{2}}\right] .
$$

Besides, when taking $\omega=\omega_{\mathrm{IN}}$, the asymptotic power is

$$
\beta\left(\omega_{\mathrm{IN}}\right)=\lim _{n, p \rightarrow \infty} \Phi\left[-z_{\alpha}+\mathbb{E}^{2}\left(u_{i}\right) \mathbb{E}\left(v_{i}{ }^{-2}\right) \frac{p^{1 / 2} n\left\{\boldsymbol{\theta}^{\mathrm{T}}\left(\boldsymbol{\theta} \boldsymbol{\theta}^{\mathrm{T}}-\boldsymbol{\theta}_{0} \boldsymbol{\theta}_{0}^{\mathrm{T}}\right) \boldsymbol{\theta}\right\}}{\sqrt{2}}\right],
$$

which equals to the maximum value of the asymptotic power in the whole family.

In addition, we will verify that under the FvML and mixed FvML distributions, the weight $\omega_{\text {IN }}$ satisfies condition $(\mathrm{C} 1)$.

When $X_{i}$ follows a FvML distribution with density function $c_{p, \kappa} \exp \left(\kappa \mathbf{x}^{\mathrm{T}} \boldsymbol{\theta}\right)$. Then, the density function of $u_{i}$ is $c_{p, \kappa}\left(1-u^{2}\right)^{\frac{p-3}{2}} \exp (\kappa u)$, where

$$
c_{p, \kappa}=1 / \int_{-1}^{1}\left(1-u^{2}\right)^{\frac{p-3}{2}} \exp (\kappa u) d u .
$$

So, we have

$$
\mathbb{E}\left(v_{i}^{-4}\right)=c_{p, \kappa} \int_{-1}^{1}\left(1-u^{2}\right)^{\frac{p-7}{2}} \exp (\kappa u) d u
$$

and

$$
\mathbb{E}\left(v_{i}^{-2}\right)=c_{p, \kappa} \int_{-1}^{1}\left(1-u^{2}\right)^{\frac{p-5}{2}} \exp (\kappa u) d u .
$$

Then, according to (S.2.7) in the supplement of [4], we have

$$
\mathbb{E}\left(v_{i}^{-4}\right)=\frac{(\kappa / 2)^{2} \Gamma\left(\frac{p-5}{2}\right) \mathcal{I}_{\frac{p}{2}-3}(\kappa)}{\Gamma\left(\frac{p-1}{2}\right) \mathcal{I}_{\frac{p}{2}-1}(\kappa)}
$$

and

$$
\mathbb{E}\left(v_{i}^{-2}\right)=\frac{(\kappa / 2) \Gamma\left(\frac{p-3}{2}\right) \mathcal{I}_{\frac{p}{2}-2}(\kappa)}{\Gamma\left(\frac{p-1}{2}\right) \mathcal{I}_{\frac{p}{2}-1}(\kappa)},
$$

where

$$
\mathcal{I}_{\nu}(\kappa) \doteq \frac{(\kappa / 2)^{\nu}}{\sqrt{\pi} \Gamma\left(\nu+\frac{1}{2}\right)} \int_{-1}^{1}\left(1-s^{2}\right)^{\nu-\frac{1}{2}} \exp (\kappa s) d s
$$


is the modified Bessel function of the first kind and of order $v$. Then, we have

$$
\frac{\mathbb{E}\left(v_{i}^{-4}\right)}{\mathbb{E}^{2}\left(v_{i}^{-2}\right)}=\frac{\Gamma\left(\frac{p-5}{2}\right) \mathcal{I}_{\frac{p}{2}-3}(\kappa) \Gamma\left(\frac{p-1}{2}\right) \mathcal{I}_{\frac{p}{2}-1}(\kappa)}{\Gamma\left(\frac{p-3}{2}\right) \mathcal{I}_{\frac{p}{2}-2}(\kappa) \Gamma\left(\frac{p-3}{2}\right) \mathcal{I}_{\frac{p}{2}-2}(\kappa)}=\frac{(p-3) \mathcal{I}_{\frac{p}{2}-3}(\kappa) \mathcal{I}_{\frac{p}{2}-1}(\kappa)}{(p-5) \mathcal{I}_{\frac{p}{2}-2}(\kappa) \mathcal{I}_{\frac{p}{2}-2}(\kappa)}
$$

Then, according to (S.2.6) and Lemma S.2.2(i) in the supplement of [4], we can obtain

$$
\begin{aligned}
\frac{\mathbb{E}\left(v_{i}^{-4}\right)}{\mathbb{E}^{2}\left(v_{i}^{-2}\right)} & =\frac{(p-3) \mathcal{I}_{\frac{p}{2}-3}(\kappa) \mathcal{I}_{\frac{p}{2}-1}(\kappa)}{(p-5) \mathcal{I}_{\frac{p}{2}-2}(\kappa) \mathcal{I}_{\frac{p}{2}-2}(\kappa)} \\
& =\frac{p-3}{p-5}\left(A_{p-2}^{2}(\kappa)+\frac{p-4}{\kappa} A_{p-2}(\kappa)\right) \\
& \leq \frac{p-3}{p-5}\left[1+\frac{p-4}{(p-4) / 2+\sqrt{\kappa^{2}+(p-4)^{2} / 4}}\right] \\
& \leq \frac{p-3}{p-5}\left[1+\frac{p-4}{p-4}\right] \\
& =O(1),
\end{aligned}
$$

for all $p>5$, where $A_{p}(\kappa) \doteq I_{p / 2}(\kappa) / I_{p / 2-1}(\kappa)$ and

$$
A_{p-2}(\kappa) \leq \frac{\kappa}{(p-4) / 2+\sqrt{\kappa^{2}+(p-4)^{2} / 4}} .
$$

When $\boldsymbol{X}_{i}$ follows the mixture of two FvML distributions with the same location parameter, the density function of $\boldsymbol{X}_{i}$ can be denoted as

$$
\lambda c_{p, \kappa_{1}} \exp \left(\kappa_{1} \mathbf{x}^{\mathrm{T}} \boldsymbol{\theta}\right)+(1-\lambda) c_{p, \kappa_{2}} \exp \left(\kappa_{2} \mathbf{x}^{\mathrm{T}} \boldsymbol{\theta}\right) .
$$

the density function of $u_{i}$ is

$$
\lambda c_{p, \kappa_{1}}\left(1-u^{2}\right)^{\frac{p-3}{2}} \exp \left(\kappa_{1} u\right)+(1-\lambda) c_{p, \kappa_{2}}\left(1-u^{2}\right)^{\frac{p-3}{2}} \exp \left(\kappa_{2} u\right) .
$$

So, we have

$$
\begin{aligned}
\mathbb{E}\left(v_{i}^{-4}\right)= & \lambda c_{p, \kappa_{1}} \int_{-1}^{1}\left(1-u^{2}\right)^{\frac{p-7}{2}} \exp \left(\kappa_{1} u\right) d u \\
& +(1-\lambda) c_{p, \kappa_{2}} \int_{-1}^{1}\left(1-u^{2}\right)^{\frac{p-7}{2}} \exp \left(\kappa_{2} u\right) d u
\end{aligned}
$$

and

$$
\begin{aligned}
\mathbb{E}\left(v_{i}^{-2}\right)= & \lambda c_{p, \kappa_{1}} \int_{-1}^{1}\left(1-u^{2}\right)^{\frac{p-5}{2}} \exp \left(\kappa_{1} u\right) d u \\
& +(1-\lambda) c_{p, \kappa_{2}} \int_{-1}^{1}\left(1-u^{2}\right)^{\frac{p-5}{2}} \exp \left(\kappa_{2} u\right) d u
\end{aligned}
$$


Then, according to (S.2.7) in the supplement of [4], we have

$$
\begin{aligned}
\mathbb{E}\left(v_{i}^{-4}\right) & =\lambda \frac{\left(\kappa_{1} / 2\right)^{2} \Gamma\left(\frac{p-5}{2}\right) \mathcal{I}_{\frac{p}{2}-3}\left(\kappa_{1}\right)}{\Gamma\left(\frac{p-1}{2}\right) \mathcal{I}_{\frac{p}{2}-1}\left(\kappa_{1}\right)}+(1-\lambda) \frac{\left(\kappa_{2} / 2\right)^{2} \Gamma\left(\frac{p-5}{2}\right) \mathcal{I}_{\frac{p}{2}-3}\left(\kappa_{2}\right)}{\Gamma\left(\frac{p-1}{2}\right) \mathcal{I}_{\frac{p}{2}-1}\left(\kappa_{2}\right)} \\
& =\lambda \frac{\kappa_{1}^{2} \mathcal{I}_{\frac{p}{2}-3}\left(\kappa_{1}\right)}{(p-3)(p-5) \mathcal{I}_{\frac{p}{2}-1}\left(\kappa_{1}\right)}+(1-\lambda) \frac{\kappa_{2}^{2} \mathcal{I}_{\frac{p}{2}-3}\left(\kappa_{2}\right)}{(p-3)(p-5) \mathcal{I}_{\frac{p}{2}-1}\left(\kappa_{2}\right)} \\
& =\frac{\lambda \kappa_{1}^{2}}{(p-3)(p-5)}\left(1+\frac{p-4}{\kappa_{1} A_{p-2}\left(\kappa_{1}\right)}\right) \\
& +\frac{(1-\lambda) \kappa_{2}^{2}}{(p-3)(p-5)}\left(1+\frac{p-4}{\kappa_{2} A_{p-2}\left(\kappa_{2}\right)}\right) \\
& \leq \frac{\lambda \kappa_{1}^{2}}{(p-3)(p-5)}\left(1+\frac{p-4}{\kappa_{1} A_{p-2}\left(\kappa_{1}\right)}\right) \\
& +\frac{(1-\lambda) \kappa_{2}^{2}}{(p-3)(p-5)}\left(1+\frac{p-4}{\kappa_{2} A_{p-2}\left(\kappa_{2}\right)}\right),
\end{aligned}
$$

where $\mathcal{I}_{\frac{p}{2}-3}(\kappa)=\mathcal{I}_{\frac{p}{2}-1}(\kappa)+\frac{p-4}{\kappa} \mathcal{I}_{\frac{p}{2}-2}(\kappa)$ due to (S.2.6) in supplement of [4].

$$
\begin{aligned}
\mathbb{E}\left(v_{i}^{-2}\right) & =\lambda \frac{\left(\kappa_{1} / 2\right) \Gamma\left(\frac{p-3}{2}\right) \mathcal{I}_{\frac{p}{2}-2}\left(\kappa_{1}\right)}{\Gamma\left(\frac{p-1}{2}\right) \mathcal{I}_{\frac{p}{2}-1}\left(\kappa_{1}\right)}+(1-\lambda) \frac{\left(\kappa_{2} / 2\right) \Gamma\left(\frac{p-3}{2}\right) \mathcal{I}_{\frac{p}{2}-2}\left(\kappa_{2}\right)}{\Gamma\left(\frac{p-1}{2}\right) \mathcal{I}_{\frac{p}{2}-1}\left(\kappa_{2}\right)} \\
& =\lambda \frac{\kappa_{1} \mathcal{I}_{\frac{p}{2}-2}\left(\kappa_{1}\right)}{(p-3) \mathcal{I}_{\frac{p}{2}-1}\left(\kappa_{1}\right)}+(1-\lambda) \frac{\kappa_{2} \mathcal{I}_{\frac{p}{2}-2}\left(\kappa_{2}\right)}{(p-3) \mathcal{I}_{\frac{p}{2}-1}\left(\kappa_{2}\right)} \\
& =\frac{\lambda \kappa_{1}}{(p-3) A_{p-2}\left(\kappa_{1}\right)}+\frac{(1-\lambda) \kappa_{2}}{(p-3) A_{p-2}\left(\kappa_{2}\right)} .
\end{aligned}
$$

Then, we have

$$
\begin{aligned}
\frac{\mathbb{E}\left(v_{i}^{-4}\right)}{\mathbb{E}^{2}\left(v_{i}^{-2}\right)} & =\frac{\frac{\lambda \kappa_{1}^{2}}{(p-3)(p-5)}\left(1+\frac{p-4}{\kappa_{1} A_{p-2}\left(\kappa_{1}\right)}\right)+\frac{(1-\lambda) \kappa_{2}^{2}}{(p-3)(p-5)}\left(1+\frac{p-4}{\kappa_{2} A_{p-2}\left(\kappa_{2}\right)}\right)}{\left[\frac{\lambda \kappa_{1}}{(p-3) A_{p-2}\left(\kappa_{1}\right)}+\frac{(1-\lambda) \kappa_{2}}{(p-3) A_{p-2}\left(\kappa_{2}\right)}\right]^{2}} \\
& \leq \frac{\frac{\lambda \kappa_{1}^{2}}{(p-3)(p-5)}\left(1+\frac{p-4}{\kappa_{1} A_{p-2}\left(\kappa_{1}\right)}\right)+\frac{(1-\lambda) \kappa_{2}^{2}}{(p-3)(p-5)}\left(1+\frac{p-4}{\kappa_{2} A_{p-2}\left(\kappa_{2}\right)}\right)}{\left[\frac{\lambda \kappa_{1}}{(p-3) A_{p-2}\left(\kappa_{1}\right)}\right]^{2}+\left[\frac{(1-\lambda) \kappa_{2}}{(p-3) A_{p-2}\left(\kappa_{2}\right)}\right]^{2}} .
\end{aligned}
$$

Because

$$
\frac{\frac{\kappa_{1}^{2}}{(p-3)(p-5)}\left(1+\frac{p-4}{\kappa_{1} A_{p-2}\left(\kappa_{1}\right)}\right)}{\left[\frac{\kappa_{1}}{(p-3) A_{p-2}\left(\kappa_{1}\right)}\right]^{2}}=O(1)
$$

and

$$
\frac{\frac{\kappa_{2}^{2}}{(p-3)(p-5)}\left(1+\frac{p-4}{\kappa_{1} A_{p-2}\left(\kappa_{2}\right)}\right)}{\left[\frac{\kappa_{2}}{(p-3) A_{p-2}\left(\kappa_{2}\right)}\right]^{2}}=O(1)
$$


Then, we have

$$
\frac{\mathbb{E}\left(v_{i}^{-4}\right)}{\mathbb{E}^{2}\left(v_{i}^{-2}\right)}=O(1)
$$

Then, we obtain the conclusion that under the FvML and mixed FvML distributions, the weight $\omega_{\text {IN }}$ satisfies condition (C1). Similarly, it is easy to verify that under the FvML and mixed FvML distributions, the weight $\omega_{\mathrm{N}}, \omega_{\mathrm{C}}, \omega_{\mathrm{R}}, \omega_{\mathrm{S}}$ satisfies condition (C1).

\section{Proof of Proposition 2.1}

Under $H_{0}$, we have that

$$
\widetilde{W}_{n} / \frac{T_{n}\left(\omega_{\mathrm{N}}\right)}{\sqrt{\sigma_{n}^{2}\left(\omega_{N}\right)}}=\sqrt{\frac{p-1}{p}} \frac{n-1}{n} \frac{b_{2}\left(\omega_{N}\right)}{n^{-1} \sum_{i=1}^{n} v_{i 0}^{2}} .
$$

Therefore, $\widetilde{W}_{n} / \frac{T_{n}\left(\omega_{\mathrm{N}}\right)}{\sqrt{\sigma_{n}^{2}\left(\omega_{N}\right)}} \rightarrow^{p} 1$ as $n, p \rightarrow \infty$, because $n^{-1} \sum_{i=1}^{n} v_{i 0}^{2} \rightarrow^{p} \mathbb{E}\left(v_{i 0}^{2}\right)=$ $b_{2}\left(\omega_{N}\right)$. Then, under Condition $(\mathrm{C} 0)$ and $H_{0}$, this leads to $\widetilde{W}_{n} / \frac{T_{n}\left(\omega_{\mathrm{N}}\right)}{\sqrt{\widehat{\sigma}_{n}^{2}\left(\omega_{N}\right)}} \rightarrow^{p} 1$ as $n, p \rightarrow \infty$, due to Theorem 3.2.

\section{Proof of Corollary 3.1}

$\boldsymbol{X}_{i}$ follows the FvML distribution, hence we have that $f(\cdot)=\exp (\cdot)$.

First, we will prove that $v_{i}$ converges to a constant with probability one. To this end, we just need to show that, under the FvML distribution, $u_{i}$ converges to $\mathbb{E}\left(u_{i}\right)$ with probability one. Specifically, due to the equation on the last line of the fourth page of [14], we have

$$
\operatorname{var}\left(u_{i}\right)=1-\frac{p-1}{\kappa} A_{p}(\kappa)-\left\{A_{p}(\kappa)\right\}^{2},
$$

where $A_{p}(\kappa) \doteq I_{p / 2}(\kappa) / I_{p / 2-1}(\kappa)$, and

$$
\mathcal{I}_{\nu}(\kappa) \doteq \frac{(\kappa / 2)^{\nu}}{\sqrt{\pi} \Gamma\left(\nu+\frac{1}{2}\right)} \int_{-1}^{1}\left(1-s^{2}\right)^{\nu-\frac{1}{2}} \exp (\kappa s) d s
$$

is the modified Bessel function of the first kind and of order $v$. Due to Lemma S.2.2(i) in the supplement of [4], we have

$$
A_{p}(\kappa) \geq G_{\frac{p}{2}, \frac{p}{2}}(\kappa) \doteq \frac{\kappa}{\frac{p}{2}+\sqrt{\kappa^{2}+\left(\frac{p}{2}\right)^{2}}} .
$$

Hence,

$$
\operatorname{var}\left(u_{i}\right)=1-\frac{p-1}{\kappa} A_{p}(\kappa)-\left\{A_{p}(\kappa)\right\}^{2}
$$




$$
\begin{aligned}
& \leq 1-\frac{p-1}{\kappa} \frac{\kappa}{\frac{p}{2}+\sqrt{\kappa^{2}+\left(\frac{p}{2}\right)^{2}}}-\frac{\kappa^{2}}{\left(\frac{p}{2}+\sqrt{\kappa^{2}+\left(\frac{p}{2}\right)^{2}}\right)^{2}} \\
& =1-\frac{p-1}{\frac{p}{2}+\sqrt{\kappa^{2}+\left(\frac{p}{2}\right)^{2}}}-\frac{\kappa^{2}}{\left(\frac{p}{2}+\sqrt{\kappa^{2}+\left(\frac{p}{2}\right)^{2}}\right)^{2}} \\
& \leq 1-\frac{p-1}{\frac{p}{2}+\sqrt{\kappa^{2}+\left(\frac{p}{2}\right)^{2}}}-\frac{\kappa^{2}}{\left(\frac{p}{2}+\sqrt{\kappa^{2}+\left(\frac{p}{2}\right)^{2}}\right)^{2}} \\
& \leq \frac{\sqrt{\kappa^{2}+\left(\frac{p}{2}\right)^{2}}-\frac{p}{2}+1}{\frac{p}{2}+\sqrt{\kappa^{2}+\left(\frac{p}{2}\right)^{2}}}-\frac{\kappa^{2}}{\left(\frac{p}{2}+\sqrt{\kappa^{2}+\left(\frac{p}{2}\right)^{2}}\right)^{2}} \\
& \leq \frac{1}{\frac{p}{2}+\sqrt{\kappa^{2}+\left(\frac{p}{2}\right)^{2}}}+\frac{\sqrt{\kappa^{2}+\left(\frac{p}{2}\right)^{2}}-\frac{p}{2}}{\frac{p}{2}+\sqrt{\kappa^{2}+\left(\frac{p}{2}\right)^{2}}}-\frac{\kappa^{2}}{\left(\frac{p}{2}+\sqrt{\kappa^{2}+\left(\frac{p}{2}\right)^{2}}\right)^{2}} \\
& \leq \frac{1}{p}+\frac{\left(\sqrt{\kappa^{2}+\left(\frac{p}{2}\right)^{2}}-\frac{p}{2}\right)\left(\frac{p}{2}+\sqrt{\kappa^{2}+\left(\frac{p}{2}\right)^{2}}\right)}{\left(\frac{p}{2}+\sqrt{\kappa^{2}+\left(\frac{p}{2}\right)^{2}}\right)^{2}} \\
& -\frac{\kappa^{2}}{\left(\frac{p}{2}+\sqrt{\kappa^{2}+\left(\frac{p}{2}\right)^{2}}\right)^{2}} \leq \frac{1}{p} \text {. }
\end{aligned}
$$

Besides, there exist a positive integer $p_{0}$ and a real constant $c$ such that

$$
\mathbb{E}\left\{u_{i}-\mathbb{E}\left(u_{i}\right)\right\}^{4} \leq c \mathbb{E}\left\{u_{i}-\mathbb{E}\left(u_{i}\right)\right\}^{2}
$$

for any $p \geq p_{0}$ and any $\kappa>0$ (see Lemma S.2.1(ii) in the supplement of [4]). Hence, $\mathbb{E}\left\{u_{i}-\mathbb{E}\left(u_{i}\right)\right\}^{4} \leq c / p^{2}$ due to (A.10). Thus, we have $\mathbb{E}\left\{u_{i}-\mathbb{E}\left(u_{i}\right)\right\}^{4}=$ $O\left(p^{-2}\right)$ and

$$
\sum_{p}^{\infty} \mathrm{P}\left(\left|u_{i}-\mathbb{E}\left(u_{i}\right)\right| \geq \epsilon\right)<\sum_{p}^{\infty} \frac{\mathbb{E}\left\{u_{i}-\mathbb{E}\left(u_{i}\right)\right\}^{4}}{\epsilon^{4}}<\infty, \forall \epsilon>0,
$$

that is, $u_{i} \rightarrow \mathbb{E}\left(u_{i}\right)$ with probability one.

Then, we have that $v_{i}$ converges to a constant with probability one due to $v_{i}=\sqrt{1-u_{i}^{2}}$, which leads to

$$
\frac{\mathbb{E}^{2}\left(u_{i}\right) \mathbb{E}^{2}\left\{\omega\left(v_{i}\right) v_{i}^{-1}\right\}}{\mathbb{E}\left\{\omega^{2}\left(v_{i}\right)\right\}} \rightarrow \mathbb{E}^{2}\left(u_{i}\right) \mathbb{E}\left(v_{i}^{-2}\right) .
$$


Hence, the asymptotic power of all the $T_{n}(\omega)$-based tests is

$$
\beta=\lim _{n, p \rightarrow \infty} \Phi\left[-z_{\alpha}+\mathbb{E}^{2}\left(u_{i}\right) \mathbb{E}\left(v_{i}^{-2}\right) \frac{p^{1 / 2} n\left\{\boldsymbol{\theta}^{\mathrm{T}}\left(\boldsymbol{\theta} \boldsymbol{\theta}^{\mathrm{T}}-\boldsymbol{\theta}_{0} \boldsymbol{\theta}_{0}^{\mathrm{T}}\right) \boldsymbol{\theta}\right\}}{\sqrt{2}}\right] .
$$

\section{Proof of Proposition 3.1}

Under $H_{0}$, since $\boldsymbol{S}_{i}=\mathbf{U}\left\{\left(\mathbf{I}_{p}-\boldsymbol{\theta}_{0} \boldsymbol{\theta}_{0}^{\mathrm{T}}\right) \boldsymbol{X}_{i}\right\}$ and $v_{i}$ are independent, so $\boldsymbol{S}_{i}$ and $R_{i}$ are also independent. We have

$$
\mathbb{E}\left(\frac{2}{n(n-1)} \sum_{i<j} \sum_{i} R_{i} R_{j} \boldsymbol{S}_{i}^{\mathrm{T}} \boldsymbol{S}_{j}\right)=0 .
$$

Then, the variance of $R_{W}$ is

$$
\begin{aligned}
& \operatorname{var}\left(\frac{2}{n(n-1)} \sum_{i<j} \sum_{i} R_{i} R_{j} \boldsymbol{S}_{i}^{\mathrm{T}} \boldsymbol{S}_{j}\right) \\
& =\frac{2}{n(n-1)} \mathbb{E}\left\{R_{i}^{2} R_{j}^{2}\left(\boldsymbol{S}_{i}^{\mathrm{T}} \boldsymbol{S}_{j}\right)^{2}\right\} \\
& =\frac{2}{n(n-1)} \mathbb{E}\left(R_{i}^{2} R_{j}^{2}\right) \mathbb{E}\left(\boldsymbol{S}_{i}^{\mathrm{T}} \boldsymbol{S}_{j}\right)^{2} \\
& =\frac{2}{n(n-1)(p-1)} \mathbb{E}\left(R_{i}^{2} R_{j}^{2}\right) \\
& =2 n^{-4} p^{-1} \sum \sum_{i \neq j} i^{2} j^{2}\{1+o(1)\} \\
& =\sigma_{n}^{2}\left(R_{W}\right)\{1+o(1)\},
\end{aligned}
$$

where $\sigma_{n}^{2}\left(R_{W}\right)=2 n^{-4} p^{-1} \sum_{i \neq j} \sum_{i} i^{2}=O\left(n^{2} p^{-1}\right)$. Similar to the proof of Theorem 3.1 , to prove the normality of $R_{W} / \sqrt{\sigma_{n}^{2}\left(R_{W}\right)}$, we only need to show

$$
\frac{\sum_{j=2}^{n} \mathbb{E}\left(Z_{n j}^{2} \mid \mathcal{F}_{n, j-1}\right)}{\sigma_{n}^{2}\left(R_{W}\right)} \stackrel{p}{\rightarrow} 1,
$$

and

$$
\mathbb{E}\left\{\sum_{j=2}^{n} \mathbb{E}\left(Z_{n j}^{4} \mid \mathcal{F}_{n, j-1}\right)\right\}=o\left\{\sigma_{n}^{4}\left(R_{W}\right)\right\},
$$

where $Z_{n i}=2 /\{n(n-1)\} \sum_{j=1}^{i-1} \boldsymbol{V}_{i}^{\mathrm{T}} \boldsymbol{V}_{j}, \boldsymbol{V}_{i}=R_{i} \boldsymbol{S}_{i}$. Let $\mathbf{A}=\mathbb{E}\left(\boldsymbol{V}_{i} \boldsymbol{V}_{i}^{\mathrm{T}}\right)$, it can be shown that

$$
\sum_{j=2}^{n} \mathbb{E}\left(Z_{n j}^{2} \mid \mathcal{F}_{n, j-1}\right)=\sum_{j=2}^{n} \mathbb{E}\left[\left\{\frac{2}{n(n-1)} \sum_{i=1}^{j-1} \boldsymbol{V}_{i}^{\mathrm{T}} \boldsymbol{V}_{j}\right\}^{2} \mid \mathcal{F}_{n, j-1}\right]
$$




$$
\begin{aligned}
& =\sum_{j=2}^{n} \frac{4}{n^{2}(n-1)^{2}} \mathbb{E}\left\{\left(\sum_{i_{1}=1}^{j-1} \sum_{i_{2}=1}^{j-1} \boldsymbol{V}_{i_{1}}^{\mathrm{T}} \boldsymbol{V}_{j} \boldsymbol{V}_{i_{2}}^{\mathrm{T}} \boldsymbol{V}_{j}\right) \mid \mathcal{F}_{n, j-1}\right\} \\
& =\sum_{j=2}^{n} \frac{4}{n^{2}(n-1)^{2}} \sum_{i_{1}=1}^{j-1} \sum_{i_{2}=1}^{j-1} \boldsymbol{V}_{i_{1}}^{\mathrm{T}} \mathbb{E}\left(\boldsymbol{V}_{j} \boldsymbol{V}_{j}^{\mathrm{T}}\right) \boldsymbol{V}_{i_{2}} \\
& =C_{n 1}+C_{n 2},
\end{aligned}
$$

where

$$
C_{n 1}=\frac{4}{n^{2}(n-1)^{2}} \sum_{j=2}^{n} \sum_{i=1}^{j-1} \boldsymbol{V}_{i}^{\mathrm{T}} \mathbf{A} \boldsymbol{V}_{i} \text { and } C_{n 2}=\frac{8}{n^{2}(n-1)^{2}} \sum_{j=2}^{n} \sum_{i_{1}<i_{2}}^{j-1} \sum_{i_{1}}^{j-1} \mathbf{A} \boldsymbol{V}_{i_{2}}
$$

Next, we consider $C_{n 1}$ and note that $\mathbf{A}=\mathbb{E}\left(R_{i}^{2}\right)\left(\mathbf{I}_{p}-\boldsymbol{\theta}_{0} \boldsymbol{\theta}_{0}^{\mathrm{T}}\right) /(p-1)$ due to Lemma A.1. Furthermore, we have

$$
\begin{aligned}
\mathbb{E}\left(C_{n 1}\right) & =\frac{2}{n(n-1)} \mathbb{E}\left(\boldsymbol{V}_{i}^{\mathrm{T}} \mathbf{A} \boldsymbol{V}_{i}\right) \\
& =\frac{2}{n(n-1)} \operatorname{tr}\left\{\mathbb{E}\left(\mathbf{A} \boldsymbol{V}_{i} \boldsymbol{V}_{i}^{\mathrm{T}}\right)\right\} \\
& =\frac{2}{n(n-1)} \operatorname{tr}\left(\mathbf{A}^{2}\right) \\
& =\sigma_{n}^{2}\left(R_{W}\right)\{1+o(1)\},
\end{aligned}
$$

where $\boldsymbol{S}_{i}=\left(\mathbf{I}_{p}-\boldsymbol{\theta}_{0} \boldsymbol{\theta}_{0}^{\mathrm{T}}\right) \boldsymbol{X}_{i} / v_{i}$ and the last equality holds due to $\mathbb{E}\left(R_{i}^{2} R_{j}^{2}\right)=$ $\mathbb{E}^{2}\left(R_{i}^{2}\right)\{1-o(1)\}$. We also obtain

$$
\begin{aligned}
\boldsymbol{S}_{i}^{\mathrm{T}} \mathbf{A} \boldsymbol{S}_{i} & \left.\left.=\frac{\mathbb{E}\left(R_{i}^{2}\right)}{v_{i}^{2}(p-1)} \boldsymbol{X}_{i}^{\mathrm{T}}\left(\mathbf{I}_{p}-\boldsymbol{\theta}_{0} \boldsymbol{\theta}_{0}^{\mathrm{T}}\right)\left(\mathbf{I}_{p}-\boldsymbol{\theta}_{0} \boldsymbol{\theta}_{0}^{\mathrm{T}}\right)\right)\left(\mathbf{I}_{p}-\boldsymbol{\theta}_{0} \boldsymbol{\theta}_{0}^{\mathrm{T}}\right)\right) \boldsymbol{X}_{i} \\
& =\frac{\mathbb{E}\left(R_{i}^{2}\right)}{(p-1)}
\end{aligned}
$$

Thus,

$$
\left(\boldsymbol{S}_{i}^{\mathrm{T}} \mathbf{A} \boldsymbol{S}_{i}\right)^{2}=\frac{\mathbb{E}^{2}\left(R_{i}^{2}\right)}{(p-1)^{2}}
$$

So, due to $\mathbb{E}\left(R_{i}^{4}\right)=O\left(n^{4}\right)$ and $\mathbb{E}\left(R_{i}^{2}\right)=O\left(n^{2}\right)$,

$$
\begin{aligned}
\operatorname{var}\left(C_{n 1}\right) & =\operatorname{var}\left(\frac{4}{n^{2}(n-1)^{2}} \sum_{j=2}^{n} \sum_{i=1}^{j-1} \boldsymbol{V}_{i}^{\mathrm{T}} \mathbf{A} \boldsymbol{V}_{i}\right) \\
& =\operatorname{var}\left\{\frac{4}{n^{2}(n-1)^{2}} \sum_{j=2}^{n} \sum_{i=1}^{j-1} \frac{\mathbb{E}\left(R_{i}^{2}\right)}{(p-1)} R_{i}^{2}\right\}
\end{aligned}
$$




$$
\begin{aligned}
& \text { Inverse norm weight spatial sign test } \\
& =\operatorname{var}\left\{\frac{4}{n^{2}(n-1)^{2}} \sum_{i=1}^{n} \sum_{j=i+1}^{n} \frac{\mathbb{E}\left(R_{i}^{2}\right)}{(p-1)} R_{i}^{2}\right\} \\
& =\operatorname{var}\left\{\frac{4}{n^{2}(n-1)^{2}} \sum_{i=1}^{n} \frac{\mathbb{E}\left(R_{i}^{2}\right)(n-i)}{(p-1)} R_{i}^{2}\right\} \\
& \leq O\left\{n^{-5}(p-1)^{-2}\right\} \mathbb{E}\left(R_{i}^{4}\right) \mathbb{E}^{2}\left(R_{i}^{2}\right) \\
& =o\left\{\sigma_{n}^{4}\left(R_{W}\right)\right\} .
\end{aligned}
$$

Then, we have $C_{n 1} / \sigma_{n}^{2}\left(R_{W}\right) \rightarrow^{p} 1$. Similarly,

$$
\begin{aligned}
\mathbb{E}\left(C_{n 2}^{2}\right) & =\mathbb{E}\left\{\frac{8}{n^{2}(n-1)^{2}} \sum_{j=2}^{n} \sum_{i_{1}<i_{2}}^{j-1} \sum_{i_{1}}^{j-1} \mathbf{A} \boldsymbol{V}_{i_{2}}\right\}^{2} \\
& =O\left(n^{-8}\right) \mathbb{E}\left(\sum_{j_{1}=2}^{n} \sum_{j_{2}=2}^{n} \sum_{i_{1}<i_{2}}^{j_{1}-1} \sum_{j_{1}-1}^{j_{2}-1} \sum_{i_{3}<i_{4}}^{j_{2}-1} \sum_{i_{1}}^{\mathrm{T}} \mathbf{A} \boldsymbol{V}_{i_{2}} \boldsymbol{V}_{i_{3}}^{\mathrm{T}} \mathbf{A} \boldsymbol{V}_{i_{4}}\right) \\
& =O\left(n^{-8}\right) \mathbb{E}\left\{\sum_{j_{1}<j_{2}}^{n} \sum_{i_{1}<i_{2}}^{j_{1}-1} \sum_{j_{1}-1}\left(\boldsymbol{V}_{i_{1}}^{\mathrm{T}} \mathbf{A} \boldsymbol{V}_{i_{2}}\right)^{2}\right\} \\
& =O\left(n^{-8}\right) \mathbb{E}\left\{\sum_{j_{2}=2 \sum_{1}=1}^{n} \sum_{i_{1}<i_{2}}^{j_{2}-1 j_{1}-1} \sum_{j_{1}-1}\left(\boldsymbol{V}_{i_{1}}^{\mathrm{T}} \mathbf{A} \boldsymbol{V}_{i_{2}}\right)^{2}\right\} \\
& =O\left(n^{-4}\right) \mathbb{E}\left\{\left(\boldsymbol{V}_{i}^{\mathrm{T}} \mathbf{A} \boldsymbol{V}_{j}\right)^{2}\right\} \\
& =O\left(n^{-4}\right) \mathbb{E}\left\{R_{i}^{2} R_{j}^{2}\left(\boldsymbol{S}_{i}^{\mathrm{T}} \mathbf{A} \boldsymbol{S}_{j}\right)^{2}\right\} \\
& =O\left(n^{-4}\right) \mathbb{E}\left(R_{i}^{2} R_{j}^{2}\right) \operatorname{tr}\left\{\mathbb{E}\left(\boldsymbol{S}_{i}^{\mathrm{T}} \mathbf{A} \boldsymbol{S}_{j} \boldsymbol{S}_{j}^{\mathrm{T}} \mathbf{A} \boldsymbol{S}_{i}\right)\right\} \\
& =O\left(n^{-4} p^{-3}\right) \mathbb{E}\left(R_{i}^{2} R_{j}^{2}\right) \mathbb{E}^{2}\left(R_{i}^{2}\right)=o\left\{\sigma_{n}^{4}\left(R_{W}\right)\right\}
\end{aligned}
$$

where $i \neq j$ in the fourth equality and we used $\mathbf{A}=(p-1)^{-1} \mathbb{E}\left(R_{i}^{2}\right)\left(\mathbf{I}_{p}-\boldsymbol{\theta}_{0} \boldsymbol{\theta}_{0}^{\mathrm{T}}\right)$ and Lemma A.1 (ii) in the seventh equality. Then (A.11) holds.

Next, we only need to show

$$
\mathbb{E}\left\{\sum_{j=2}^{n} \mathbb{E}\left(Z_{n j}^{4} \mid \mathcal{F}_{n, j-1}\right)\right\}=o\left\{\sigma_{n}^{4}\left(R_{W}\right)\right\} .
$$

Note that

$$
\begin{aligned}
\mathbb{E}\left\{\sum_{j=2}^{n} \mathbb{E}\left(Z_{n j}^{4} \mid \mathcal{F}_{n, j-1}\right)\right\} & =\sum_{j=2}^{n} \mathbb{E}\left\{\mathbb{E}\left(Z_{n j}^{4} \mid \mathcal{F}_{n, j-1}\right)\right\} \\
& =\sum_{j=2}^{n} \mathbb{E}\left(Z_{n j}^{4}\right)
\end{aligned}
$$




$$
\begin{aligned}
& =\sum_{j=2}^{n} \mathbb{E}\left(\left(\frac{2}{n(n-1)} \sum_{j=1}^{i-1} \boldsymbol{V}_{i}^{\mathrm{T}} \boldsymbol{V}_{j}\right)^{4}\right) \\
& =O\left(n^{-8}\right) \sum_{j=2}^{n} \mathbb{E}\left(\sum_{i=1}^{j-1} \boldsymbol{V}_{j}^{\mathrm{T}} \boldsymbol{V}_{i}\right)^{4}
\end{aligned}
$$

can be decomposed as $Q+P$, where

$$
\begin{aligned}
& Q=O\left(n^{-8}\right) \sum_{j=2}^{n} \sum_{i_{1}<i_{2}}^{j-1} \sum_{j}^{j-1} \mathbb{E}\left(\boldsymbol{V}_{j}^{\mathrm{T}} \boldsymbol{V}_{i_{1}} \boldsymbol{V}_{i_{1}}^{\mathrm{T}} \boldsymbol{V}_{j} \boldsymbol{V}_{j}^{\mathrm{T}} \boldsymbol{V}_{i_{2}} \boldsymbol{V}_{i_{2}}^{\mathrm{T}} \boldsymbol{V}_{j}\right), \\
& P=O\left(n^{-8}\right) \sum_{j=2}^{n} \sum_{i=1}^{j-1} \mathbb{E}\left\{\left(\boldsymbol{V}_{j}^{\mathrm{T}} \boldsymbol{V}_{i}\right)^{4}\right\} .
\end{aligned}
$$

Because

$$
\begin{aligned}
Q & =O\left(n^{-5}\right) \mathbb{E}\left(\boldsymbol{V}_{j}^{\mathrm{T}} \boldsymbol{V}_{i_{1}} \boldsymbol{V}_{i_{1}}^{\mathrm{T}} \boldsymbol{V}_{j} \boldsymbol{V}_{j}^{\mathrm{T}} \boldsymbol{V}_{i_{2}} \boldsymbol{V}_{i_{2}}^{\mathrm{T}} \boldsymbol{V}_{j}\right) \\
& =O\left(n^{-5}\right) \operatorname{tr}\left\{\mathbb{E}\left(\boldsymbol{V}_{i_{1}} \boldsymbol{V}_{i_{1}}^{\mathrm{T}} \boldsymbol{V}_{j} \boldsymbol{V}_{j}^{\mathrm{T}} \boldsymbol{V}_{i_{2}} \boldsymbol{V}_{i_{2}}^{\mathrm{T}} \boldsymbol{V}_{j} \boldsymbol{V}_{j}^{\mathrm{T}}\right)\right\} \\
& =O\left(n^{-5}\right) \operatorname{tr}\left[\mathbb{E}\left\{\mathbb{E}\left(\boldsymbol{V}_{i_{1}} \boldsymbol{V}_{i_{1}}^{\mathrm{T}} \boldsymbol{V}_{j} \boldsymbol{V}_{j}^{\mathrm{T}} \boldsymbol{V}_{i_{2}} \boldsymbol{V}_{i_{2}}^{\mathrm{T}} \boldsymbol{V}_{j} \boldsymbol{V}_{j}^{\mathrm{T}} \mid \boldsymbol{V}_{j}\right)\right\}\right] \\
& =O\left(n^{-5}\right) \operatorname{tr}\left\{\mathbb{E}\left(\mathbf{A} \boldsymbol{V}_{j} \boldsymbol{V}_{j}^{\mathrm{T}} \mathbf{A} \boldsymbol{V}_{j} \boldsymbol{V}_{j}^{\mathrm{T}}\right)\right\} \\
& =O\left(n^{-5}\right) \mathbb{E}\left\{\left(\boldsymbol{V}_{j}^{\mathrm{T}} \mathbf{A} \boldsymbol{V}_{j}\right)^{2}\right\},
\end{aligned}
$$

$Q=O\left\{n^{-5}(p-1)^{-2}\right\} \mathbb{E}\left(R_{j}^{4}\right) \mathbb{E}^{2}\left(R_{i}^{2}\right)=o\left\{\sigma_{n}^{4}\left(R_{W}\right)\right\}$ due to (A.13). Similarly, due to Lemma A.1 (iii), we can show that

$$
\begin{aligned}
P & =O\left(n^{-6}\right) \mathbb{E}\left\{\left(\boldsymbol{V}_{j}^{\mathrm{T}} \boldsymbol{V}_{i}\right)^{4}\right\} \\
& =O\left(n^{-6}\right) \mathbb{E}\left\{\left(R_{i}^{4} R_{j}^{4}\right)\left(\boldsymbol{S}_{j}^{\mathrm{T}} \boldsymbol{S}_{i}\right)^{4}\right\} \\
& =O\left(n^{-6}\right) \mathbb{E}\left(R_{i}^{4} R_{j}^{4}\right) \mathbb{E}\left\{\left(\boldsymbol{S}_{j}^{\mathrm{T}} \boldsymbol{S}_{i}\right)^{4}\right\} \\
& =O\left(n^{-6} p^{-2}\right) \mathbb{E}\left(R_{i}^{4} R_{j}^{4}\right) \\
& =o\left\{\sigma_{n}^{4}\left(R_{W}\right)\right\} .
\end{aligned}
$$

So, we have complete the proof of $R_{W} / \sqrt{\sigma_{n}^{2}\left(R_{W}\right)}$. Similarly, $R_{S} / \sqrt{\sigma_{n}^{2}\left(R_{S}\right)}$ is also asymptotically standard normal. Finally, we obtain the conclusion.

\section{References}

[1] Arnold, R., And Jupp, P. E. Statistics of orthogonal axial frames. Biometrika 100, 3 (2013), 571-586. MR3094438

[2] Briggs, M. S. Dipole and quadrupole tests of the isotropy of gamma-ray burst locations. The Astrophysical Journal 407, 1 (1993), 126-134.

[3] Chang, T., And Rivest, L. M-estimation for location and regression parameters in group models: A case study using stiefel manifolds. Annals of Statistics 29, 3 (2001), 784-814. MR1865341 
[4] Cutting, C., Paindaveine, D., and Verdebout, T. Testing uniformity on high-dimensional spheres against monotone rotationally symmetric alternatives. Annals of Statistics 45 (2017), 1024-1058. MR3662447

[5] Downs, T. D. Spherical regression. Biometrika 90, 3 (2003), 655-668. MR2006842

[6] Feng, L., Liu, B., And Ma, Y. An inverse norm sign test of location parameter for high-dimensional data. Journal of Business $\&$ Economic Statistics (2020). MR4272937

[7] Fisher, N. I. Problems with the current definitions of the standard deviation of wind direction. Journal of Applied Meteorology 26, 11 (1987), $1522-1529$.

[8] Garciaportugues, E., Paindaveine, D., and Verdebout, T. On optimal tests for rotational symmetry against new classes of hyperspherical distributions. Journal of the American Statistical Association (2019), 1-15. MR4189764

[9] Hall, P. G., And Heyde, C. C. Martingale limit theory and its application. Journal of the American Statistical Association 78, 381 (1983). MR0755100

[10] Jupp, P. E., Fisher, N. I., Lewis, T., And Embleton, B. J. J. Statistical analysis of spherical data. Biometrics 44, 2 (1987), 592. MR0899958

[11] Larsen, P. V., Blaesild, P., And Sorensen, M. K. Improved likelihood ratio tests on the von mises-fisher distribution. Biometrika 89, 4 (2002), 947-951. MR1946523

[12] Ley, C., Paindaveine, D., And Verdebout, T. High-dimensional tests for spherical location and spiked covariance. Journal of Multivariate Analysis 139 (2015), 79-91. MR3349481

[13] Ley, C., Swan, Y., Thiam, B., and Verdebout, T. Optimal restimation of a spherical location. Statistica Sinica 23, 1 (2013), 305-332. MR3076169

[14] Ley, C., And Verdebout, T. Local powers of optimal one-sample and multi-sample tests for the concentration of fisher-von mises-langevin distributions. International Statistical Review 82, 3 (2014), 440-456. MR3280985

[15] Ley, C., And Verdebout, T. Modern directional statistics. New York: Chapman \& Hall/CRC interdisciplinary statistics, 2017. MR3752655

[16] Marc, H., AND DAvy, P. Semiparametrically efficient rank-based inference for shape. i. optimal rank-based tests for sphericity. Ann. Statist 34, 6, 2707-2756.

[17] Paindaveine, D., And Verdebout, T. Optimal rank-based tests for the location parameter of a rotationally symmetric distribution on the hypersphere. Working Papers ECARES (2013). MR3380740

[18] Paindaveine, D., and Verdebout, T. On high-dimensional sign tests. Bernoulli 22, 3 (2016), 1745-1769. MR3474832

[19] Paindaveine, D., and Verdebout, T. Inference on the mode of weak directional signals: A le cam perspective on hypothesis testing near singularities. Annals of Statistics 45, 2 (2017), 800-832. MR3650401

[20] Paindaveine, D., And Verdebout, T. Detecting the direction of a sig- 
nal on high-dimensional spheres: Non-null and le cam optimality results. Probability Theory and Related Fields (2019), 1-52. MR4087491

[21] Rivest, L. Spherical regression for concentrated fisher-von mises distributions. Annals of Statistics 17, 1 (1989), 307-317. MR0981452

[22] Storetvedt, K. M., And Scheidegger, A. E. Orthogonal joint systems in the bergen area, southwest norway, and their regional significance. Physics of the Earth and Planetary Interiors 73 (1992), 255-263.

[23] Watson, G. S. Statistics on spheres. Wiley, New York, 1983. MR0709262 